\title{
Diversidad y conservación de los mamíferos mayores de Loreto, Perú
}

\author{
Diversity and conservation of the large mammals from Loreto, Peru
}

Silvia Diaz* 1

https://orcid.org/0000-0002-9344-4991

silvia.diaz2@unmsm.edu.pe

Pamela Sánchez-Vendizú ${ }^{1,2}$

https://orcid.org/0000-0002-3374-6031

p.sanchez.vendizu@gmail.com

Laura Graham-Angeles ${ }^{1}$

https://orcid.org/0000-0003-1966-6534

laura.graham@unmsm.edu.pe

Víctor Pacheco ${ }^{1,3}$

https://orcid.org/0000-0002-1005-135X

vpachecot@unmsm.edu.pe

\section{*Corresponding author}

1 Universidad Nacional Mayor de San Marcos, Museo de Historia Natural, Apartado 14-0434, Lima-15072, Perú.

2 Programa de Pos-Graduação em Zoología, Universidade Federal do Pará, Museu Paraense Emilio Goeldi, Brazil.

3 Universidad Nacional Mayor de San Marcos, Facultad de Ciencias Biológicas, Institutode Ciencias Biológicas "Antonio Raimondi", Lima, Perú.

\section{Citación}

Diaz S, Sánchez-Vendizú P, Graham-Angeles L, Pacheco V. 2021. iversidad y conservación de los mamíferos mayores de Loreto, Perú Revista peruana de biología 28(especial): e21910 001- 034 (Diciembre 2021). doi: http://dx.doi.org/10.15381/rpb.v28iespecial. 21910

$\begin{array}{ll}\text { Presentado: } & 09 / 02 / 2021 \\ \text { Aceptado: } & 10 / 08 / 2021 \\ \text { Publicado online: } & 30 / 12 / 2021\end{array}$

Editor:

Leonardo Romero

\section{Resumen}

En el presente trabajo, presentamos una lista actualizada de mamíferos mayores para Loreto compuesta por 72 especies en 8 órdenes y 25 familias. Esta lista fue elaborada a partir de la minuciosa revisión de bases de datos, colecciones zoológicas y publicaciones científicas. En Loreto se encuentran 5 especies endémicas nacionales: Lagothrix flavicauda, Saguinus (Leontocebus) illigeri, Saguinus (Leontocebus) nigrifrons, Pithecia aequatorialis y Pithecia isabela, siendo las tres últimas también endémicas para Loreto. Se encontraron 292 localidades diferentes a partir de 3286 registros de mamíferos mayores. Se presenta un mapa de distribución de las localidades analizadas remarcando ecorregiones y registros históricos (antes del 2001) y contemporáneos (después del 2001). Asimismo, se presentan mapas con la distribución de las localidades donde han sido registradas las 72 especies estudiadas, y un mapa de densidad de las localidades registradas, el cual permite identificar dos zonas con vacíos de información: 1) la zona Datem del Marañón-Loreto y 2) la zona Alto Amazonas-Ucayali. Estas zonas requieren evaluaciones urgentes para complementar el conocimiento de la biodiversidad de Loreto y sustentar adecuadamente los planes de conservación y desarrollo sustentable.

\section{Abstract}

In this work, we present an updated list of large mammals for Loreto composed of 72 species in 8 orders and 25 families. This list is elaborated from review of databases, zoological collections, and scientific publications. In Loreto is registered five Peruvian endemic species: Lagothrix flavicauda, Saguinus (Leontocebus) illigeri, Saguinus (Leontocebus) nigrifrons, Pithecia aequatorialis and Pithecia isabela; the last three have presence only in Loreto. We found 292 different locations and 3286 records of large mammals and build a distribution map highlighting ecoregions and historical (before 2001) and contemporary (after 2001) records. Likewise, we build maps for each of the 72 species with records in Loreto, and a density map that reveal two areas with information gaps: 1) the Datem del Marañón-Loreto zone, and 2) the Alto Amazonas-Ucayali zone. These zones require urgent evaluations to complement knowledge of Loreto's biodiversity and adequately support conservation and sustainable development plans.

Palabras clave:

Amazonia; distribución; endemismo; Mammalia; Selva baja.

Keywords:

Amazonia; Distribution; Endemism; Mammalia; lowland forest. 


\section{Introducción}

El Perú es uno de los cinco países con mayor diversidad de mamíferos, estimándose que existen no menos de 573 especies de mamíferos a lo largo del territorio peruano (Pacheco et al. 2020, 2021). Esta diversidad es más alta en la selva baja tropical (Voss \& Emmons 1996; Emmons \& Feer 1997; Pacheco 2002; Pacheco et al. 2009, 2020); no obstante, se conoce poco sobre la diversidad de mamíferos a nivel regional. Loreto es el departamento más extenso del Perú, ubicado en la Amazonia occidental y ocupa el $28.7 \%$ del territorio nacional con una extensión de $368851 \mathrm{Km}^{2}$, mayor que varios países de la región Neotropical, y semejante en área a Alemania; sin embargo, su biodiversidad de mamíferos mayores es poco conocida, limitando la toma de decisiones en cuanto a las prioridades de conservación y de desarrollo sostenible. Pitman et al. (2013) estimaron 267 especies de mamíferos para este departamento basándose en mapas de distribución de la IUCN y la lista de Pacheco et al. (2009). De estas especies, 17 fueron consideradas amenazadas a nivel internacional (3 EN y $14 \mathrm{VU}$ ) y 3 amenazadas a nivel nacional, dando un total de 20 especies de mamíferos amenazados.

El presente trabajo documenta por primera vez la diversidad de mamíferos mayores para el departamento de Loreto en base a la revisión de bases de datos de museos nacionales e internacionales y literatura especializada. El trabajo proporciona 1) Una lista de especies, señalándose las especies endémicas, raras, comunes y listadas en alguna categoría de conservación; 2) un mapa de las localidades analizadas, remarcando ecorregiones y registros históricos y contemporáneos; 3) mapas de distribución de las localidades donde han sido registradas cada especie y 4) un mapa de densidad donde se señalan los vacíos de información.

\section{Material y métodos}

Área de estudio. - El departamento de Loreto se localiza en la zona noreste del Perú, aproximadamente entre los paralelos $0^{\circ} 2^{\prime} 5^{\prime \prime}$ - $8^{\circ} 42^{\prime} 6^{\prime \prime} \mathrm{S}$ y $77^{\circ} 47^{\prime} 14^{\prime \prime}$ - 69 $69^{\circ} 41^{\prime \prime} \mathrm{W}$. Tiene una extensión territorial de $368851 \mathrm{Km}^{2}$ al $28.7 \%$ del territorio nacional, donde predomina el Bosque Húmedo Amazónico (sensu Britto 2017). Su superficie es bastante plana, con elevaciones entre de 70 y 220 m, excepto en la región de la Cordillera Escalera donde puede llegar a una altura de $2000 \mathrm{~m}$ aproximadamente. Loreto presenta un clima cálido y húmedo con abundantes precipitaciones pluviales. En Loreto, el río Ucayali y el río Marañón forman el gran río Amazonas. Los ríos Morona, Pastaza, Tigre y Huallaga contribuyen al río Marañón antes de formar el río Amazonas. El río Napo es afluente del río Amazonas y el río Putumayo que limita a Loreto con Colombia, desemboca en el río Amazonas ya en territorio brasileño. En Loreto se encuentran 11 áreas naturales protegidas que ocupan el $20 \%$ del territorio loretano (71988.12 $\mathrm{Km}^{2}$ ): el Parque Nacional Cordillera Azul, PN Güeppi Sekime, PN Sierra del Divisor, PN Yaguas, Reserva Nacional Allpahuayo Mishana, RN Matsés, RN Pacaya Samiria, RN Pucacuro, Reserva Comunal Airo Pai, RC Humeki y Zona Reservada Santiago-Comaina; así como también numerosas comunidades nativas, bosques de producción permanente y concesiones.

Datos y análisis. - Para elaborar la lista de especies se tomó como punto de partida los artículos de Pacheco et al. $(2009,2020,2021)$. Se consideró como mamíferos mayores a aquellas especies con un peso mayor a un kilogramo y cuyo método de muestreo sea principalmente censos u observaciones. Se consultaron libros especializados, publicaciones científicas y bases de datos disponibles en portales especializados (GBIF, https://www. gbif.org/; VerNet, http://vertnet.org/) que incluyen información de las colecciones del American Museum of Natural History, New York (AMNH); Field Museum of Natural History, Chicago (FMNH); Museum of Vertebrate Zoology, University of California, Berkeley (MVZ); Louisiana State University, Museum of Zoology, Baton Rouge (LSUMZ); Natural History Museum of Los Angeles County, Los Angeles (LACM); Smithsonian National Museum of Natural History, Washington D.C. (USNM); Museum of Texas Tech University, Lubbock (TTU); Natural History Museum of Kansas University, Lawrence (KU) y University of Michigan Museum of Zoology Ann Arbor (UMMZ). A nivel nacional, se revisó la base de datos de la colección del Museo de Historia Natural, Universidad Nacional Mayor de San Marcos, Lima (MUSM). Las coordenadas de las localidades fueron obtenidas de Stephens y Traylor (1983) y Patton et al. (2015) cuando no figuraban en los datos del espécimen. Para la nomenclatura y arreglo taxonómico se siguió a Gardner (2008), Patton et al. (2015) y Pacheco et al. $(2009,2020)$, agregándose algunos cambios taxonómicos recientes (Acosta et al. 2020).

Se elaboró un mapa con las localidades analizadas en las diferentes ecorregiones para Loreto descritas por Britto (2017), diferenciándose registros históricos y contemporáneos. Los mapas de distribución de las localidades donde han sido registradas cada especie fueron elaborados en ArcMap 10.4.1 (ESRI, Redlands, CA, USA) (Anexo 2). Se produjo también un mapa de densidad en base a las localidades registradas (Fig. 3) con el método de densidad de Kernel considerando celdas de $3 \mathrm{~km}$ y un radio de $25 \mathrm{~km}$ para una mejor visualización de las áreas con mayores localidades muestreadas y determinar las áreas que contienen vacíos de información.

El estado de conservación de las especies corresponde a la lista roja de la Unión Internacional para la Conservación de la Naturaleza (https://www.iucnredlist.org/) y la legislación peruana (D.S. 004-2014). La información referente a nivel de endemismo se basó en Pacheco et al. (2020). Con esta información se establecieron las siguientes categorías: endémica nacional $(\mathrm{N})$ y endémica del departamento de Loreto (R); siendo $\mathrm{N}$ aquella que es endémica para el territorio peruano, estando presente en Loreto y otros departamentos. Subjetivamente, se consideró a una especie como rara cuando había sido registrada en por lo menos tres (1\%) de las localidades analizadas; y común cuando había sido registrada en más de 58 (20\%). 


\section{Resultados}

Diversidad. - A partir de las revisiones, el recuento de mamíferos mayores para Loreto es de 72 especies, distribuidas en 25 familias y 47 géneros (Tabla 1) representando el $12.57 \%$ del total de mamíferos registrados para Perú (573 especies, Pacheco et al. 2021), confirmando así la alta diversidad de mamíferos existente en la selva baja (Pacheco 2002).

Se encontraron en total 3286 registros de mamíferos mayores (1462 en colecciones científicas y 1824 en literatura publicada) los cuales fueron clasificados como históricos y contemporáneos poniendo como limite el año 2001: 2044 registros fueron considerados históricos (1899-2000), 1074 contemporáneos (2001-2020) y 168 registros como históricos y contemporáneos (ambos) por haber sido registrados años antes y después del 2001 en un solo estudio (Fig. 1) detectando finalmente 292 localidades diferentes (Anexo 1) distribuidas mayormente a lo largo de los principales ríos y capitales. A partir de esto, se puede resaltar que los primates son el grupo con mayor número de localidades, seguidos por artiodáctilos y carnívoros; mientras que sirenios y cingulados son los órdenes con menor número de localidades (Fig. 2).

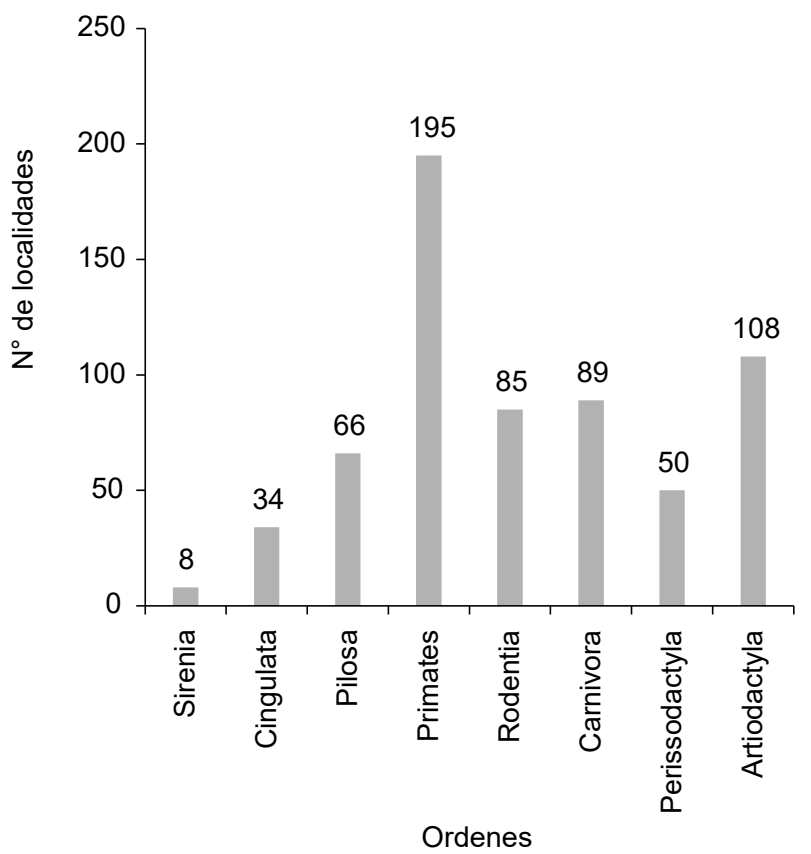

Figura 2 Número de localidades registradas por órdenes de mamíferos mayores de Loreto.

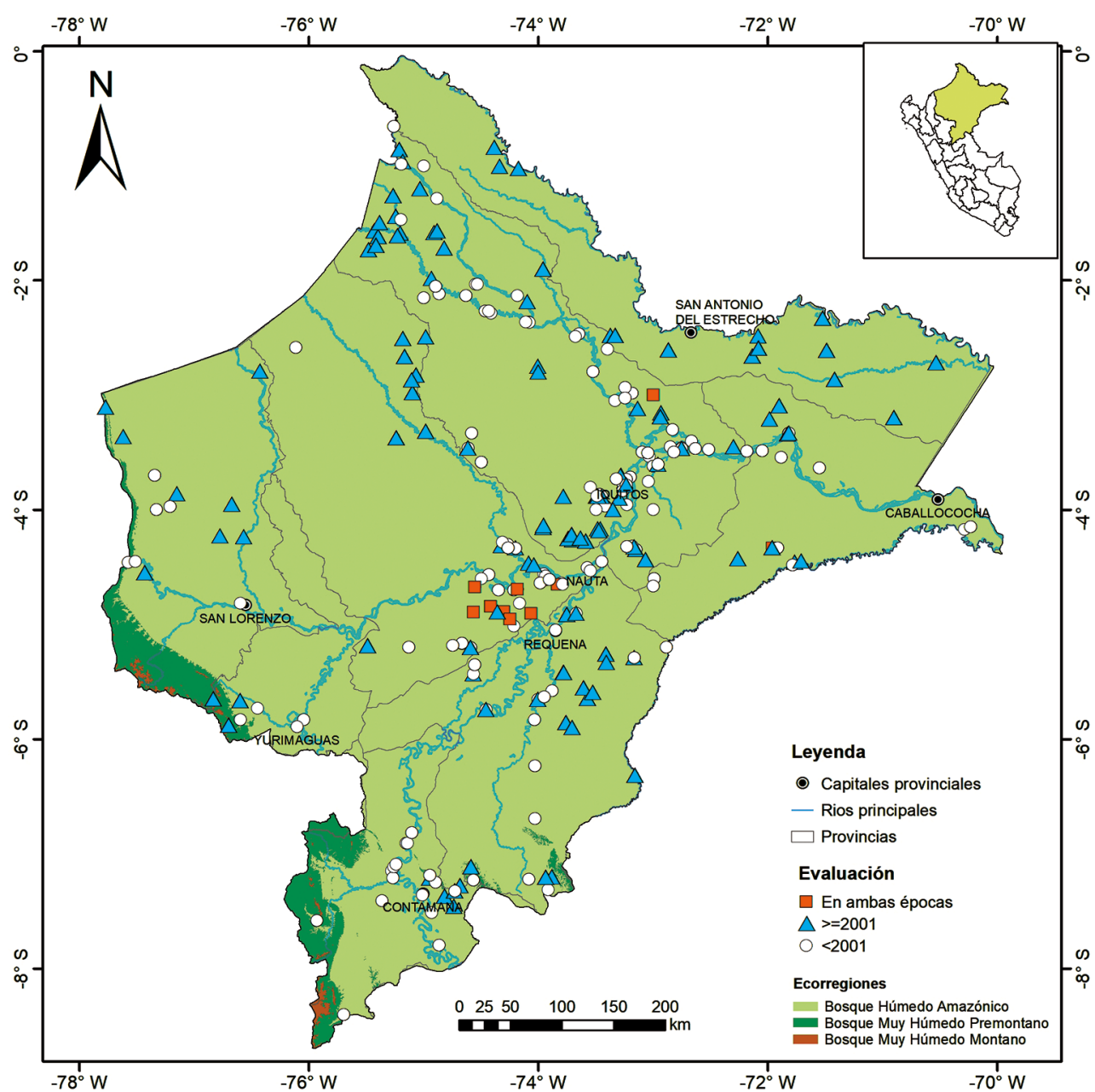

Figura 1 Mapa de distribución de las localidades analizadas de los registros de mamíferos mayores mostrando las ecorregiones descritas por Britto (2017) en Loreto. Los círculos blancos representan registros históricos (1899-2001), los triángulos celestes representan registros contemporáneos (2001-2020) y los cuadrados anaranjados representan aquellos que fueron registrados antes y después del 2001 en un solo estudio. 
Loreto contiene tres de las 15 ecorregiones descritas por Britto (2017) (Fig. 1): el Bosque Húmedo Amazónico que abarca la mayor parte del departamento y donde se encuentran la mayor cantidad de las localidades analizadas (288) y riqueza de especies (73); el Bosque Muy Húmedo Premontano con solo 3 localidades y 22 especies; y el Bosque Muy Húmedo Montano con una sola localidad y una menor riqueza de especies (14) por comprender una pequeña porción de territorio loretano.

Asimismo, el 73\% de las localidades analizadas se encontraron fuera de las Áreas Naturales Protegidas (ANP) por el Estado y fue donde se encontró evidentemente la mayor riqueza de especies (70 especies); mientras que el $27 \%$ de localidades restantes se encontraron distribuidas de la siguiente manera: Parque Nacional Cordillera Azul (1), Parque Nacional Sierra del Divisor (11), Parque Nacional Yaguas (3), Reserva Nacional Allpahuayo Mishana (7), Reserva Nacional Matsés (10), Reserva Nacional Pacaya Samiria (34), Reserva Nacional Pucacuro (8), Reserva Comunal Airo Pai (2), Zona Reservada Santiago Comaina (2). Por otro lado, el PN Pacaya Samiria presentó la mayor riqueza con 39 especies; mientras que la RN Allpahuayo Mishana presentó la menor riqueza con 9 especies. Sin embargo, estos últimos valores se ven afectados por las dificultades de acceso a distintas áreas dentro de las ANPs, las cuales limitan las observaciones y muestreos.

Las provincias que albergan mayor riqueza de especies son Maynas (66), Requena (57) y Ucayali (54); mientras que las que tienen menor riqueza son Alto Amazonas (23) y Datem del Marañón (24). Estos valores podrían estar reflejando un mayor esfuerzo de muestreo en zonas con fácil acceso y cerca de las ciudades.

Se elaboraron mapas de distribución de la presencia en las localidades registradas para cada una de las especies (Anexo 2), así como también un índice de localidades por especie (Anexo 3). Con esta información se puede considerar a las especies como comunes a aquellas registradas en más del $20 \%$ de las localidades registradas: Cebus (Sapajus) apella macrocephalus, Alouatta seniculus, Dicotyles tajacu, Lagothrix lagothricha poeppigii y Saimiri sciureus macrodon; mientras que aquellas registradas en menos del 1\% de las localidades registradas pueden ser consideradas como raras: Lagothrix flavicauda, Tremarctos ornatus, Saguinus (Leontocebus) nigricollis graellsi, Aotus nigriceps, Coendou bicolor, Coendou ichillus y Dinomys branickii.

Especies endémicas. - De los mamíferos mayores registrados para Loreto, cinco especies son endémicas de Perú: el mono choro de cola amarilla Lagothrix flavicauda, el pichico pardo Saguinus (Leontocebus) illigeri, el pichico de frente negra Saguinus (Leontocebus) nigrifrons, el huapo ecuatorial Pithecia aequatorialis y el huapo de Isabel Pithecia isabela, estas tres últimas especies están presentes sólo en Loreto. En la actualidad es escasa la información referente a estas especies.

Conservación. - Se registran 15 especies (en 7 órdenes) protegidas por alguna categoría de amenaza. La
IUCN lista a las siguientes especies como En Peligro Crítico (CR): Lagothrix flavicauda; En Peligro (EN): Ateles belzebuth, Ateles chamek, Lagothrix lagothricha poeppigii, Inia geoffrensis y Pteronura brasiliensis; Vulnerable (VU): Aotus nancymae, Cacajao calvus ucayalii, Callimico goeldii, Lagothrix lagothricha lagothricha, Myrmecophaga tridactyla, Priodontes maximus, Tapirus terrestris, Tayassu pecari, Tremarctos ornatus y Trichechus inunguis. Además, ocho especies se encuentran en situación Casi Amenazada (NT): Atelocynus microtis, Cebus (Cebus) albifrons yuracus, Leopardus wiedii, Lontra longicaudis, Panthera onca, Saguinus (Leontocebus) nigricollis graellsi, Saguinus (Leontocebus) tripartitus y Speothos venaticus.

Trece especies están categorizadas como amenazadas por la legislación peruana (D.S. 004-2014), incluyendo a Lagothrix flavicauda como En Peligro Crítico (CR); cuatro especies En Peligro (EN): Ateles belzebuth, Ateles chamek, Lagothrix lagothricha lagothricha y Pteronura brasiliensis y cuatro especies en situación de Casi Amenazada (NT): Panthera onca, Puma concolor, Tapirus terrestris y Tayassu pecari.

De todas las especies protegidas, Lagothrix flavicauda es la especie más amenazada al estar categorizada como En Peligro Crítico (CR), seguida por Ateles belzebuth, Ateles chamek, Lagothrix lagothricha lagothricha y Pteronura brasiliensis catalogadas En Peligro (EN) por la IUCN y la Legislación Nacional actual debido principalmente a la cacería intensiva de la que fueron objeto y perdida de hábitat; por lo que su protección debería ser una prioridad.

Vacíos de información. - La región de Loreto presenta algunas zonas con muy pocos o ningún registro de mamíferos, y son consideradas aquí como Vacíos de Información (Fig. 3): 1) la zona Datem del Marañón-Loreto y 2) la zona Alto Amazonas-Ucayali. Se sugiere dar prioridad a estas zonas en estudios de diversidad y conservación.

\section{Discusión}

El presente trabajo reúne información de diversas fuentes para estimar diversidad y la distribución geográfica de los mamíferos mayores dentro de Loreto, los cuales son especies importantes por los roles que cumplen en los ecosistemas, principalmente como dispersores de semillas y reforestación de los bosques. Como resultado, Loreto es un departamento altamente diverso en mamíferos mayores, además esta información permitió elaborar por primera vez una tabla con todos los registros encontrados de las especies de mamíferos mayores por provincias y localidades (Tabla 1) y mapas de distribución para cada especie (Anexo 2).

Pitman et al. (2013) reportaron 71 especies de mamíferos mayores para Loreto en condición de "registrados" en base a los mapas de la IUCN y la lista de Pacheco et al. (2009) e incluyeron a Aotus miconax, Cuniculus taczanowskii, Leopardus colocolo, Leopardus tigrinus, Lycalopex culpaeus y Odocoileus peruvianus. Sin embargo, estas especies no han sido incluidas aquí porque su presencia no está sustentada por alguna publicación o váucher de 
colección científica (Pacheco et al. 2020). Aun esto, es probable la presencia de algunas de estas especies en las zonas inexploradas de la Cordillera Escalera, como el musmuqui andino Aotus miconax (Patterson \& LópezWong 2014). Por otro lado, la diversidad de mamíferos mayores reportada por Quintana et al. (2009) para el departamento Ucayali es muy similar a la reportada aquí para Loreto, debido en parte porque ambos departamentos están conformados mayoritariamente por Bosque Húmedo Amazónico.

Resalta que los primates fueron el grupo taxonómico con el mayor número de localidades analizadas, seguidas por los artiodáctilos y carnívoros (Figura 2), lo cual se debería a que son animales más visibles y carismáticos, lo cual al parecer se ha reflejado en mayor interés y oportunidades de financiamiento. Por esta razón, recomendamos más investigaciones sobre aquellos grupos menos estudiados, pero no por eso menos importantes, como los sirenios y cingulados, los cuales tienen pocos registros presentes en pocas localidades.

La Figura 3 muestra dos vacíos de información, los cuales se encuentran dentro de las provincias que resultaron con menor cantidad de registros. Esto es expli- cable debido a que estas se encuentran alejadas de las principales ciudades y son de difícil acceso, por lo que la cantidad de reportes hechos por expediciones científicas en dichas zonas es baja. Los mapas de distribución para cada especie (Anexo 2) facilitan la evaluación de sus ocurrencias, las cuales son cruciales para su conservación, especialmente en aquellas con alguna categoría de amenaza o datos deficientes. Esperamos que esta información contribuya a la toma de decisiones adecuadas en distintos niveles, sobre todo enfocada en aquellas especies en actual estado de amenaza (VU, EN, CR) y también en aquellas que están cerca de estarlo (NT).

Por último, debe resaltarse que la fuente principal de estos registros son las colecciones científicas, y que levantar considerablemente la data de registros requiere el fortalecimiento de las instituciones como el Instituto de Investigaciones de la Amazonia Peruana (IIAP) y la Universidad Nacional de la Amazonia Peruana (UNAP) que lideran el desarrollo de inventarios biológicos de Loreto y la capacitación de biólogos y conservacionistas locales; pero también de la participación de museos, universidades e institutos de investigación nacionales, con amplia experiencia en diversidad a nivel nacional.

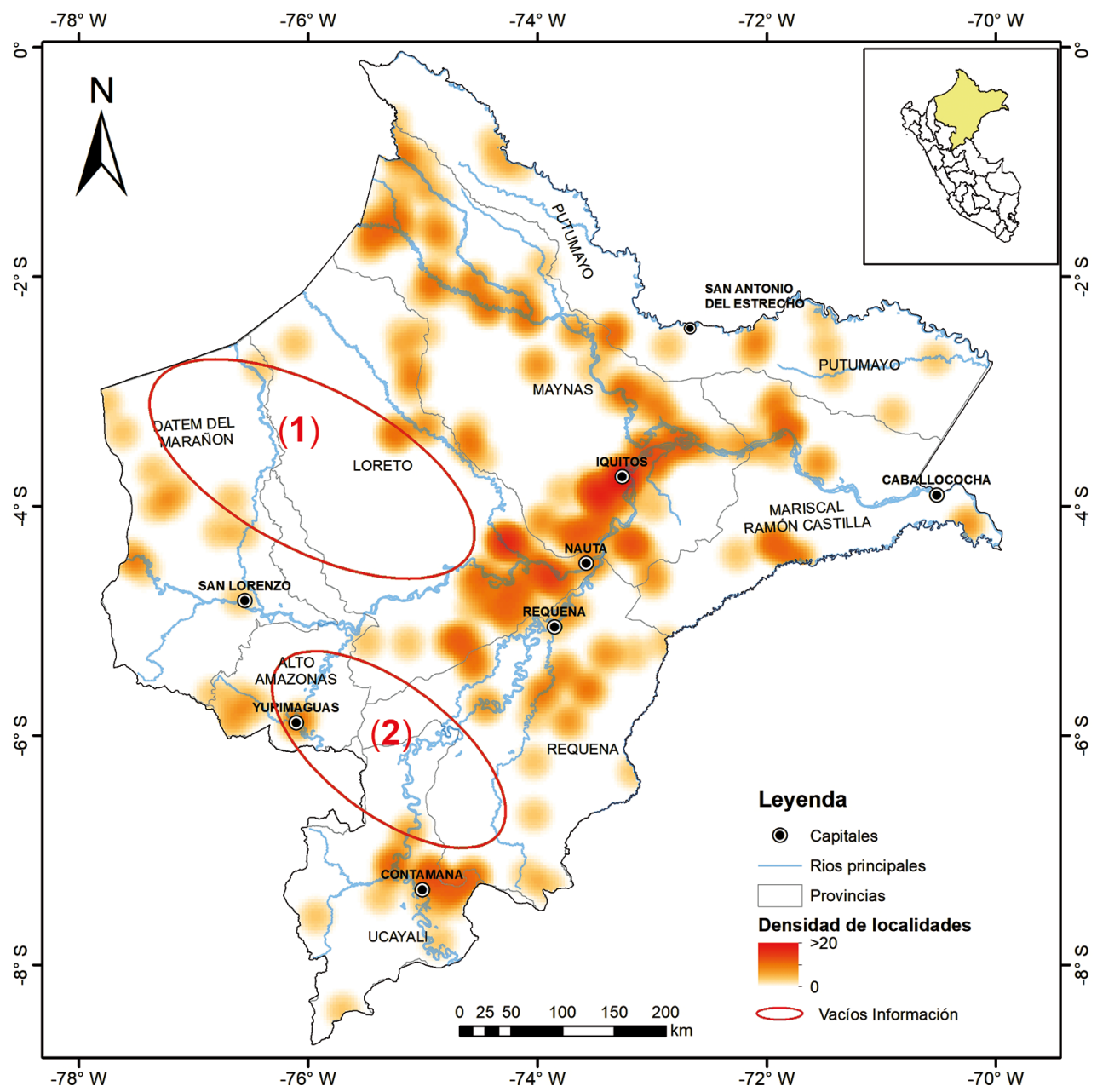

Figura 3 Mapa de calor de Loreto para las localidades analizadas indicando sus densidades de registros de mamíferos mayores. Las elipses rojas señalan los vacios de informacion encontrados 1) la zona Datem del Marañón-Loreto y 2) la zona Alto Amazonas-Ucayali. 


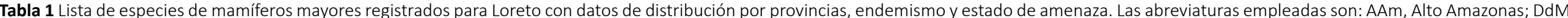

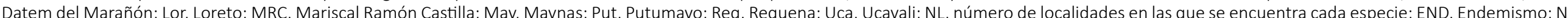

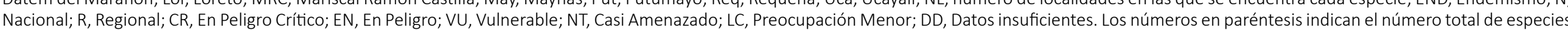

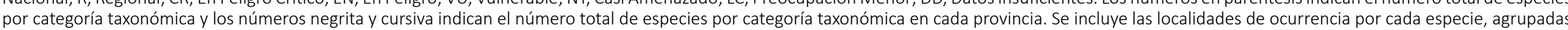

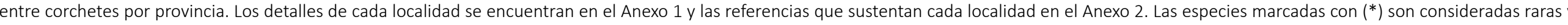
con $\left(^{* *}\right)$ comunes para el departamento de Loreto.

\begin{tabular}{|c|c|c|c|c|c|c|c|c|c|c|c|c|c|c|c|}
\hline & Nombre científico & Nombre común & $\frac{\varepsilon}{4}$ & $\sum_{0}$ & ta & $\stackrel{\breve{c}}{\Sigma}$ & $\sum^{\frac{\pi}{2}}$ & 苛 & $\underset{\mathscr{g}}{\mathscr{g}}$ & さ & Localidades & $\vec{z}$ & ¿ & ż & בั่ \\
\hline & \multicolumn{15}{|l|}{ Sirenia (1) } \\
\hline & Trichechidae (1) & & & & & & & & & & & & & & \\
\hline \multirow[t]{3}{*}{1} & Trichechus inunguis (Natterer, 1883) & Manatí & & & 1 & 1 & 1 & & 1 & & {$[199,251][85][38,97,122][224,250]$} & 8 & & VU & \\
\hline & \multicolumn{15}{|l|}{ Cingulata (4) } \\
\hline & \multicolumn{15}{|l|}{ Dasypodidae (2) } \\
\hline 2 & Dasypus novemcinctus Linnaeus, 1758 & Armadillo de nueve bandas, carachupa & 1 & & 1 & 1 & 1 & 1 & 1 & & $\begin{array}{l}{[256,258][55,225][173][3,10,12,122,142,} \\
273][37,52,62][221,234,241,255,261,290, \\
291,292]\end{array}$ & 22 & & LC & \\
\hline \multirow[t]{2}{*}{3} & Dasypus pastasae (Thomas, 1901) & Armadillo de Pastaza, carachupa & & & 1 & & 1 & 1 & 1 & & {$[55][273][52][234,255,261]$} & 6 & & LC & \\
\hline & Chlamyphoridae (2) & & & & & & & & & & & & & & \\
\hline 4 & Cabassous unicinctus (Linnaeus, 1758) & Armadillo de cola desnuda & & & & 1 & 1 & & 1 & & {$[99][108,122,134,151,273][234,241,261]$} & 9 & & LC & \\
\hline \multirow[t]{3}{*}{5} & Priodontes maximus (Kerr, 1792) & Armadillo gigante, carachupa mama & 1 & & 1 & 1 & 1 & 1 & 1 & & $\begin{array}{l}{[256][55,176,232][54,71][102,273][37,52,53,} \\
56,62,76][234,241,255,261,291]\end{array}$ & 19 & & vu & \\
\hline & Pilosa (6) & & & & & & & & & & & & & & \\
\hline & Bradypodidae (1) & & & & & & & & & & & & & & \\
\hline \multirow[t]{2}{*}{6} & Bradypus variegatus infuscatus Wagler, 1831 & Perezoso de tres dedos, pelejo & 1 & 1 & 1 & 1 & 1 & 1 & 1 & 1 & $\begin{array}{l}{[254][114][205,207,208,210,212,213,216,} \\
222,223,228][71,99][38,68,75,100,104,122, \\
134,136,273][76][219,234,261][263]\end{array}$ & 28 & & LC & \\
\hline & Choloepodidae (2) & & & & & & & & & & & & & & \\
\hline 7 & Choloepus didactylus (Linnaeus, 1758) & Perezoso de dos dedos, pelejo blanco & & 1 & 1 & & 1 & 1 & 1 & & $\begin{array}{l}{[144][172,191][75,117,122,133,137,180,273]} \\
{[50][239,292]}\end{array}$ & 13 & & LC & \\
\hline \multirow[t]{2}{*}{8} & Choloepus hoffmanni Peters, 1858 & Perezoso de dos dedos de Hoffmann & & & & 1 & 1 & & 1 & 1 & {$[99][1,38,102,104,122][234][263]$} & 8 & & $\mathrm{LC}$ & \\
\hline & Cyclopedidae (1) & & & & & & & & & & & & & & \\
\hline \multirow[t]{2}{*}{9} & Cyclopes ida Thomas, 1900 & Serafín, intipelejo & & & & & 1 & & 1 & 1 & {$[68,122,138,273][234][263,289]$} & 7 & & $\mathrm{LC}$ & \\
\hline & Myrmecophagidae (2) & & & & & & & & & & & & & & \\
\hline 10 & Myrmecophaga tridactyla Linnaeus, 1758 & Oso hormiguero, oso bandera & & & 1 & 1 & 1 & 1 & 1 & 1 & $\begin{array}{l}{[55][71,177][3,104,122,273][37,50,52,62]} \\
{[230,234,241,260,261][276,288]}\end{array}$ & 18 & & $\mathrm{vu}$ & \\
\hline
\end{tabular}




\begin{tabular}{|c|c|c|c|c|c|c|c|c|c|c|c|c|c|c|c|c|}
\hline & & Nombre científico & Nombre común & $\frac{\xi}{4}$ & $\sum_{\mathrm{O}}$ & ذَ & $\stackrel{\dddot{q}}{\Sigma}$ & $\sum^{\frac{\pi}{2}}$ & $\stackrel{\vec{z}}{2}$ & $\underset{\mathscr{g}}{\mathscr{\Psi}}$ & $\stackrel{\coprod}{5}$ & Localidades & $\mathbf{z}$ & $\underset{\mathrm{E}}{\mathrm{Z}}$ & ¿ & 荾 \\
\hline 11 & & Tamandua tetradactyla (Linnaeus, 1758) & Shihui, oso hormiguero amazónico & & & 1 & 1 & 1 & 1 & 1 & 1 & $\begin{array}{l}{[55,192,205,207,208,210,212,216,222,225} \\
228][99][1,3,10,38,64,75,104,107,109,117 \\
122,142,273][56][233,234,241,245,261,274 \\
290,291][263]\end{array}$ & 35 & & LC & \\
\hline & \multicolumn{2}{|c|}{ Primates (30) } & & & & & & & & & & & & & & \\
\hline & \multicolumn{2}{|c|}{ Cebidae (17) } & & & & & & & & & & & & & & \\
\hline 12 & & Callimico goeldii (Thomas, 1904) & Supay pichico, pichico falso de Goeldi & & & 1 & & 1 & 1 & 1 & 1 & {$[61,65][122,134][52][224,273,291][281,289]$} & 10 & & vu & \\
\hline 13 & & Cebuella niveiventris Lönnberg, 1940 & Leoncito, tití de vientre blanco & & 1 & 1 & 1 & 1 & & 1 & 1 & $\begin{array}{l}{[193][61,65,229][99,198][15,19,109,110,} \\
122,124,128,159][230,234,241,247,262][263]\end{array}$ & 20 & & LC & \\
\hline 14 & & Cebuella pygmaea (Spix, 1823) & Leoncito, tití pigmeo, tití enano & & & & 1 & 1 & 1 & & & {$[54][3,24,38,59,75,100][42,50,52,56,62]$} & 12 & & LC & \\
\hline 15 & & Saguinus (Leontocebus) fuscicollis Spix, 1823 & Pichico común & & & & & & & 1 & & {$[244,247,252]$} & 3 & & LC & \\
\hline 16 & & Saguinus (Leontocebus) illigeri (Pucheran, 1845) & Pichico pardo de Illiger & 1 & & 1 & & 1 & & 1 & 1 & $\begin{array}{l}{[248,256][205,208,209,210,212,215,216,218} \\
220,222,225,228,229,232][123][185,237,240 \\
247][263,271,280,283,287,289]\end{array}$ & 27 & $\mathrm{~N}$ & LC & \\
\hline 17 & & $\begin{array}{l}\text { Saguinus (Leontocebus) lagonotus (Jiménez de la } \\
\text { Espada, 1870) }\end{array}$ & Pichico de dorso rojo & & 1 & 1 & & 1 & & & & $\begin{array}{l}{[193][61,65,88,172,178,189][15,28,29,31} \\
35,38,57,60,95,104,113,115,117,122,125 \\
128,135,147,150,155,159,161]\end{array}$ & 29 & & LC & \\
\hline \multirow[t]{2}{*}{18} & & $\begin{array}{l}\text { Saguinus (Leontocebus) nigricollis graellsi (Jiménez de } \\
\text { la Espada, 1870) }\end{array}$ & Pichico de manto negro de Graell & & & & & 1 & & & & {$[3,4]$} & $2^{*}$ & & NT & \\
\hline & & $\begin{array}{l}\text { Saguinus (Leontocebus) nigricollis nigricollis (Spix, } \\
1823 \text { ) }\end{array}$ & Pichico de manto negro & & & & 1 & 1 & 1 & & & $\begin{array}{l}{[54,71,81,84][24,38,73,75,92,100][37,42,} \\
50,52,53,56,62,76]\end{array}$ & 18 & & LC & \\
\hline 19 & & Saguinus (Leontocebus) nigrifrons (I. Geoffroy, 1850) & Pichico de frente negra & & & & 1 & 1 & & 1 & & $\begin{array}{l}{[82,99,149,177,198,206][96,109,110,118,} \\
168,180][233,234,239,245,241,255,259,261]\end{array}$ & 20 & $\mathrm{R}$ & LC & \\
\hline 20 & & $\begin{array}{l}\text { Saguinus (Leontocebus) tripartitus (Milne-Edwards, } \\
1878 \text { ) }\end{array}$ & Pichico de manto dorado & & & & & 1 & & & & $\begin{array}{l}{[9,10,11,12,13,16,19,22,25,26,27,28,30,34,} \\
35,36,38,39]\end{array}$ & 18 & & NT & \\
\hline 21 & & Saguinus (Tamarinus) mystax (Spix, 1823) & $\begin{array}{l}\text { Pichico de barba blanca, pichico cer- } \\
\text { vecero }\end{array}$ & & & 1 & 1 & 1 & & 1 & 1 & $\begin{array}{l}{[199,231][99,106,111,112,177,198,206][91,} \\
96,109,110,118,168,180][233,234,239,241 \\
245,247,255,259,261,291][263,271,278,280 \\
283]\end{array}$ & 31 & & LC & \\
\hline \multirow[t]{2}{*}{22} & & Cebus (Cebus) albifrons unicolor Spix, 1823 & $\begin{array}{l}\text { Machín frontiblanco de Spix, machín } \\
\text { blanco, mono blanco }\end{array}$ & & & 1 & 1 & 1 & & 1 & 1 & $\begin{array}{l}{[199,205,207,208,209,210,212,215,216,218,} \\
220,222,225,229][99,173,174,198][109,168] \\
{[221,230,233,234,237,239,241,245,247,261,} \\
290,292][263,278,280,287,289]\end{array}$ & 37 & & LC & \\
\hline & & Cebus (Cebus) albifrons yuracus Hershkovitz, 1949 & $\begin{array}{l}\text { Machín frontiblanco del Marañón, } \\
\text { machín blanco, mono blanco }\end{array}$ & & 1 & 1 & 1 & 1 & 1 & & & $\begin{array}{l}{[114,144,193][55,61,65,88,163,195][54,71]} \\
{[1,3,10,12,15,19,24,28,31,35,38,75,95,104} \\
107,122,150,155,159,161][37,42,50,52,53 \\
62,76]\end{array}$ & 38 & & NT & \\
\hline
\end{tabular}




\begin{tabular}{|c|c|c|c|c|c|c|c|c|c|c|c|c|c|c|c|}
\hline & Nombre científico & Nombre común & $\frac{\varepsilon}{4}$ & $\sum_{\mathrm{O}}$ & ذِ & $\underset{\check{q}}{\Sigma}$ & $\sum^{\frac{\pi}{2}}$ & $\vec{z}$ & ס্ّ̋ & Ј & Localidades & $\mathbf{z}$ & 롬 & $\underline{\underline{z}}$ & 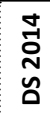 \\
\hline 23 & Cebus (Sapajus) apella macrocephalus Spix, 1823 & $\begin{array}{l}\text { Machín negro, mono negro, capuchino } \\
\text { negro }\end{array}$ & 1 & 1 & 1 & 1 & 1 & 1 & 1 & 1 & $\begin{array}{l}{[246,248,256][114,193][55,61,65,80,88,163,} \\
172,195,199,205,207,208,209,210,212,215, \\
216,218,220,222,225,228,229,231,232][54, \\
99,173,174,177,198][15,28,31,32,35,38,59, \\
95,104,107,122,150,155,159,161,168][37,52, \\
56,62][221,230,233,234,237,239,241,245, \\
247,255,260,261,274,290,292,293][263,276, \\
278,280,283,287]\end{array}$ & $78^{* *}$ & & LC & \\
\hline 24 & Aotus nancymae Hershkovitz, 1983 & $\begin{array}{l}\text { Musmuqui, mono nocturno de Nancy } \\
\text { Ma }\end{array}$ & & & 1 & 1 & 1 & & 1 & 1 & $\begin{array}{l}{[162,163,165,172,176,179,205,208,209,212,} \\
215,216,218,220,222,225,229][99,177,198] \\
{[96,100,109,122,145][234,241,247,261][263,} \\
280,283]\end{array}$ & 32 & & VU & \\
\hline 25 & Aotus nigriceps Dollman, 1909 & $\begin{array}{l}\text { Musmuqui, mono nocturno cabecine- } \\
\text { gro }\end{array}$ & & & & & & & 1 & 1 & : & $2^{*}$ & & LC & \\
\hline 26 & Aotus vociferans (Spix, 1823) & Musmuqui, mono nocturno vociferante & & & & 1 & 1 & 1 & & & $\begin{array}{l}{[54][6,15,19,24,31,38,41,46,51,66,75,78} \\
94,104,117,122,125,127,128,130,146,159] \\
{[42,52,53,56,76]}\end{array}$ & 28 & & LC & \\
\hline 27 & Saimiri boliviensis peruviensis Hershkovitz, 1984 & $\begin{array}{l}\text { Mono ardilla peruano, mono fraile, } \\
\text { frailecillo }\end{array}$ & & & 1 & 1 & 1 & & 1 & 1 & $\begin{array}{l}{[199,205,207,208,209,210,212,215,216,218,} \\
220,222,225,228,231,232][198][122][237, \\
247,260][263,280,289]\end{array}$ & 24 & & LC & \\
\hline 28 & Saimiri sciureus macrodon Elliot, 1907 & Fraile, frailecillo mono ardilla, huasa & 1 & 1 & 1 & 1 & 1 & 1 & 1 & 1 & $\begin{array}{l}{[249][58,144,193][55,61,65,88,163,166,172,} \\
176][54,71,99,149,173,177,198][3,10,12, \\
15,19,24,28,31,32,35,38,75,95,96,104,109, \\
110,116,117,122,128,150,155,159,161,168] \\
{[37,42,50,52,53,56,62,76][230,234,239,241,} \\
245,255,260,261][281,287,289]\end{array}$ & $64^{* *}$ & & LC & \\
\hline & Pitheciidae (8) & & & & & & & & & & & & & & \\
\hline 29 & Callicebus (Plecturocebus) cupreus (Spix, 1823) & Tocón cobrizo & & & & 1 & 1 & & 1 & 1 & $\begin{array}{l}{[99,149,173,177,198][108,109,110,122,168,} \\
180][224,233,234,239,241,245,247,255,261 \\
274][263,281]\end{array}$ & 23 & & LC & \\
\hline 30 & Callicebus discolor (I. Geoffroy y Deville, 1848) & Tocón colorado, tocón rojo & & 1 & 1 & & 1 & 1 & & 1 & $\begin{array}{l}{[193][61,65,88,163,170,172,210][1,10,12,} \\
15,19,28,29,31,35,38,75,95,104,107,122 \\
150,155,159,161][50,53][287,289]\end{array}$ & 31 & & LC & \\
\hline 31 & Callicebus lucifer Thomas, 1914 & Tocón negro, tocón de collar & & & 1 & 1 & 1 & 1 & & & $\begin{array}{l}{[88][54,71][1,3,24,38,44,59,75,95,100,107} \\
148,150,155,158,159,160,161][37,42,45,50 \\
52,53,56,62,76]\end{array}$ & 29 & & LC & \\
\hline 32 & Cacajao calvus ucayalii (Thomas, 1928) & Huapo colorado, mono inglés, uacari & & & & 1 & 1 & & 1 & 1 & $\begin{array}{l}{[99,173,174,177,186,198][122,169][234,247,} \\
275,279,291,292][263,278,280,283]\end{array}$ & 18 & & $\mathrm{VU}$ & \\
\hline 33 & Pithecia aequatorialis Hershkovitz, 1987 & Huapo ecuatorial, huapo negro & & & 1 & & 1 & & & & $\begin{array}{l}{[61,65,88][15,28,29,95,104,107,122,150} \\
155,159,161]\end{array}$ & 14 & $\mathrm{R}$ & LC & \\
\hline 34 & Pithecia isabela Marsh, 2014 & Huapo de Isabel & & & 1 & & & & 1 & & $\begin{array}{l}{[199,205,207,208,209,210,212,215,216,218,} \\
220,222,225,226,228,229,232][237]\end{array}$ & 18 & $\mathrm{R}$ & $\mathrm{DD}$ & \\
\hline
\end{tabular}




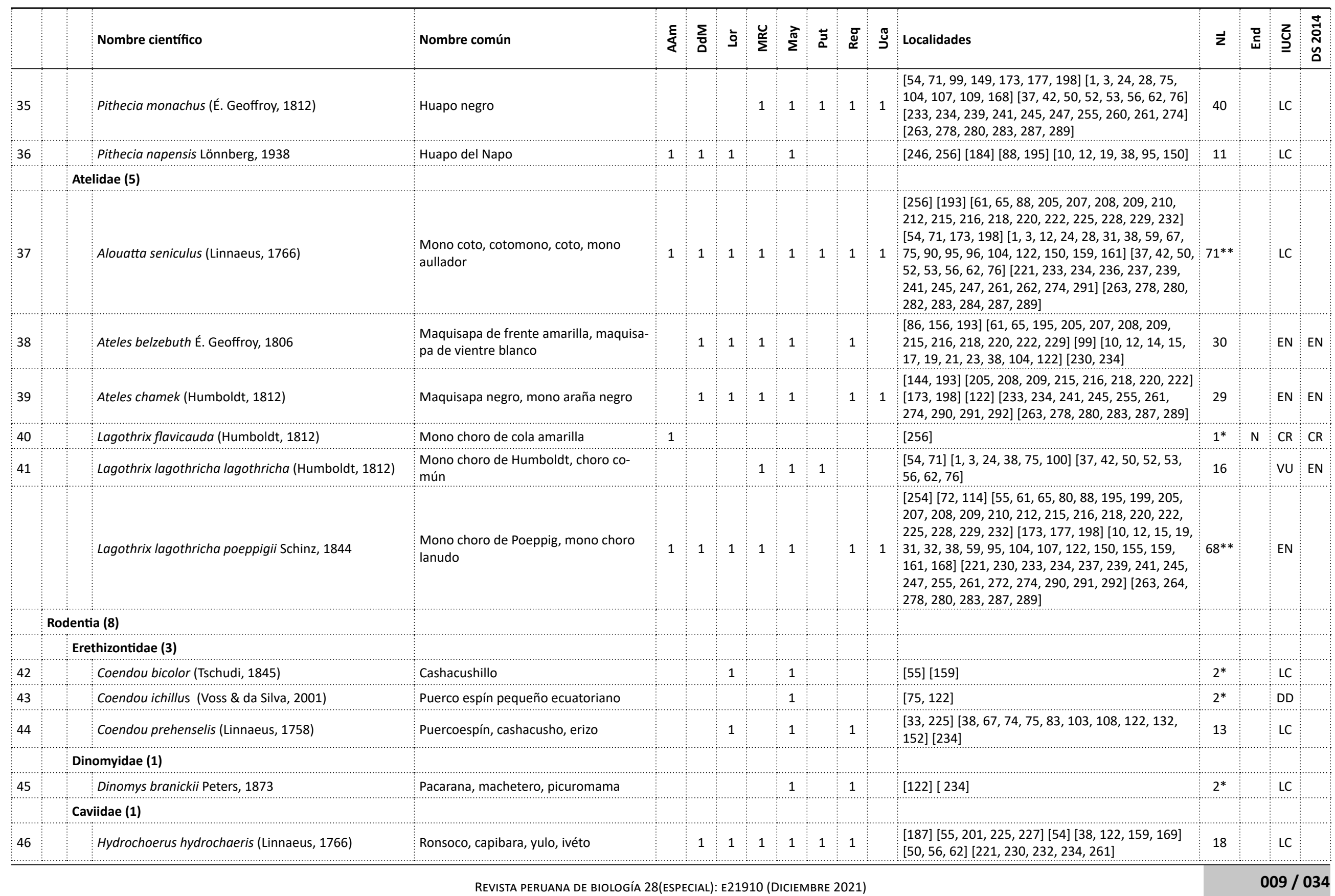




\begin{tabular}{|c|c|c|c|c|c|c|c|c|c|c|c|c|c|c|c|c|}
\hline & & Nombre científico & Nombre común & $\frac{\varepsilon}{\&}$ & $\sum_{0}$ & ᄒ্ড & $\underset{\check{c}}{\stackrel{\Psi}{\Sigma}}$ & $\sum^{\frac{\pi}{m}}$ & 苛 & $\underset{\mathscr{\varpi}}{\mathscr{g}}$ & Ј & Localidades & $\vec{z}$ & 胥 & ż & 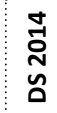 \\
\hline & \multicolumn{2}{|r|}{ Dasyproctidae (2) } & & & & & & & & & & & & & & \\
\hline 47 & & Dasyprocta fuliginosa Wagler, 1832 & Añuje, cutpe, agutí, chapana & 1 & & 1 & 1 & 1 & 1 & 1 & 1 & $\begin{array}{l}{[157,246,248,253,256][55,207,208,209,210,} \\
216,222,227,232][54,71,173,175,190][1,3, \\
10,12,38,40,59,67,69,98,104,122,131,141, \\
159,169,205][37,50,52,56,62,76][221,230, \\
233,234,239,241,245,255,261,290,291][263, \\
289]\end{array}$ & 55 & & LC & \\
\hline \multirow[t]{2}{*}{48} & & Myoprocta pratti Pocock, 1913 & Punchana, añuje menor & & 1 & 1 & 1 & 1 & 1 & 1 & 1 & $\begin{array}{l}{[143][49,55,201][71,99,153,175][3,10,38} \\
47,64,83,90,93,101,103,104,108,122,159 \\
167][37,50,52,56,76][219,230,233,234,274] \\
{[263,269,289]}\end{array}$ & 36 & & LC & \\
\hline & \multicolumn{2}{|r|}{ Cuniculidae (1) } & & & & & & & & & & & & & & \\
\hline \multirow[t]{3}{*}{49} & & Cuniculus paca (Linnaeus, 1766) & Majáz, picuro & 1 & 1 & 1 & 1 & 1 & 1 & 1 & 1 & $\begin{array}{l}{[246,248,256][143,214][49,55,79,201,205,} \\
208,216,222,225,232][54,71,173,175][3,10, \\
12,38,67,98,104,108,122,141,154,159,169] \\
{[37,52,53,56,62,76][221,233,234,239,241,} \\
255,261,290,291,292][264,269,289]\end{array}$ & 51 & & LC & \\
\hline & \multicolumn{2}{|c|}{ Carnivora (16) } & & & & & & & & & & & & & & \\
\hline & \multicolumn{2}{|r|}{ Felidae (5) } & & & & & & & & & & & & & & \\
\hline 50 & & Leopardus pardalis (Linnaeus, 1758) & Tigrillo, ocelote & 1 & 1 & 1 & 1 & 1 & 1 & 1 & 1 & $\begin{array}{l}{[248][144,187][55,207,225,228,232][99,177]} \\
{[3,5,10,12,18,74,97,122,159][37,50,52,62]} \\
{[230,234,261,292][263,268,289]}\end{array}$ & 30 & & LC & \\
\hline 51 & & Leopardus wiedii (Schinz, 1821) & Margay, huamburushu, tigrillo pequeño & 1 & & 1 & & 1 & 1 & 1 & & {$[246,233][55][10,100,159][76][230]$} & 8 & & NT & $\mathrm{DD}$ \\
\hline 52 & & Puma concolor (Linnaeus, 1771) & Puma, lluichupuma, león, tigre colorado & 1 & & 1 & 1 & 1 & 1 & 1 & 1 & $\begin{array}{l}{[248][55,211,232][54][12,122,159][52,56]} \\
{[234,255][268,289]}\end{array}$ & 14 & & LC & NT \\
\hline 53 & & Puma yagouaroundi (É. Geoffroy Saint-Hilaire, 1803) & Yaguarundi, pantera negra & 1 & & 1 & & 1 & 1 & 1 & 1 & {$[257][55][122,126,159][52][234][289]$} & 8 & & LC & \\
\hline \multirow[t]{2}{*}{54} & & Panthera onca (Linnaeus, 1758) & Otorongo, jaguar & 1 & & 1 & & 1 & 1 & 1 & 1 & $\begin{array}{l}{[246,248,256][55,205,208,211,216,222,232]} \\
{[3,97,107,119,122,159][37,50,52,56,62,76]} \\
{[234,241,255,261,290,291,292][263,265,266} \\
288,289]\end{array}$ & 34 & & NT & NT \\
\hline & \multicolumn{2}{|r|}{ Canidae (2) } & & & & & & & & & & & & & & \\
\hline 55 & & Atelocynus microtis (Sclater, 1883) & Perro de orejas cortas & & & 1 & & 1 & 1 & & 1 & {$\left[\begin{array}{l}{[55,197,205,208,216,222,232][3,10,12,122]} \\
{[37,52][286]}\end{array}\right.$} & 14 & & NT & \\
\hline \multirow[t]{2}{*}{56} & & Speothos venaticus (Lund, 1842) & Perro de monte, sachaperro & & & 1 & 1 & 1 & 1 & 1 & 1 & {$[209][190][94,122,159,169][37][241][289]$} & 9 & & NT & \\
\hline & & Ursidae (1) & & & & & & & & & & & & & & \\
\hline \multirow[t]{2}{*}{57} & & Tremarctos ornatus (F. G. Cuvier, 1825) & Oso de anteojos & & & & & & & & 1 & [287] & $1^{*}$ & & vu & \\
\hline & & Mustelidae (4) & & & & & & & & & & & & & & \\
\hline 58 & & Lontra longicaudis (Olfers, 1818) & Lobo pequeño de río, nutria & & & 1 & & 1 & 1 & 1 & & $\begin{array}{l}{[55][38,122,142,159][37,53,62][234,241,} \\
291]\end{array}$ & 11 & & NT & \\
\hline
\end{tabular}


DIVERSIDAD Y CONSERVACIÓN DE LOS MAMÍFEROS MAYORES DE LORETO

\begin{tabular}{|c|c|c|c|c|c|c|c|c|c|c|c|c|c|c|c|c|}
\hline & & Nombre científico & Nombre común & $\frac{\xi}{\alpha}$ & $\sum_{0}$ & ذَ & 災 & $\sum^{\frac{\pi}{n}}$ & 苛 & $\underset{\mathscr{\Psi}}{\mathscr{\Psi}}$ & Ј & Localidades & $\dot{z}$ & 홉 & ż & 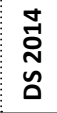 \\
\hline 59 & & Pteronura brasiliensis (Gmelin, 1788) & Lobo grande de río, nutria grande & & & 1 & 1 & 1 & 1 & 1 & & $\begin{array}{l}{[55][54][12,38,97,100,122][37,52,53,56,76]} \\
{[224,234]}\end{array}$ & 14 & & EN & EN \\
\hline 60 & & Eira barbara (Linnaeus, 1758) & Manco, tejón, taira, omayro & & 1 & 1 & 1 & 1 & 1 & 1 & 1 & $\begin{array}{l}{[144][55,201,205,207,208,210,216,222,228]} \\
{[54,99][12,38,75,100,122,142,155,159][37,} \\
50,52,56,62][233,234,239,241,245,261,290 \\
291][263,287,289]\end{array}$ & 36 & & LC & \\
\hline \multirow[t]{2}{*}{61} & & Galictis vittata (Schreber, 1776) & Hurón grande, grisón & & & 1 & & 1 & & 1 & & {$[55][122,142,159][234]$} & 5 & & LC & \\
\hline & & Procyonidae (4) & & & & & & & & & & & & & & \\
\hline 62 & & Bassaricyon alleni Thomas, 1880 & Olingo, chosna & & & & 1 & 1 & 1 & 1 & 1 & $\begin{array}{l}{[54][41,66,75,78,107,117,122,130,159][37]} \\
{[234,272][268,289]}\end{array}$ & 15 & & LC & \\
\hline 63 & & Nasua nasua (Linnaeus, 1766) & $\begin{array}{l}\text { Coatí de cola anillada, achuni, mishas- } \\
\text { ho, capiso }\end{array}$ & 1 & 1 & 1 & 1 & 1 & 1 & 1 & 1 & $\begin{array}{l}{[256][144][55,205,207,208,209,210,216,222,} \\
225,228,231][173,177,190][3,10,12,38,100, \\
104,122,159,169][37,50,52,53,56,76][221 \\
230,234,239,241,245,255,260,291][263,288 \\
289]\end{array}$ & 43 & & LC & \\
\hline 64 & & Potos flavus (Schreber, 1774) & Chosna & & & 1 & 1 & 1 & 1 & 1 & 1 & $\begin{array}{l}{[49,205,208,209,211,215,216,220,222,225,} \\
228,231][99][38,41,66,75,78,107,117,130, \\
142,155,159][37,52,56,62][234,241,255,261, \\
274,291,292][269,289]\end{array}$ & 37 & & LC & \\
\hline \multirow[t]{3}{*}{65} & & Procyon cancrivorus (G. [Baron] Cuvier, 1798) & Perro conchero, mapache cangrejero & 1 & & 1 & & 1 & 1 & 1 & & $\begin{array}{l}{[246][205,207,208,216,222][10,122,128,159]} \\
{[37,52][230,234]}\end{array}$ & 14 & & LC & \\
\hline & \multicolumn{2}{|c|}{ Perissodactyla (1) } & & & & & & & & & & & & & & \\
\hline & & Tapiridae (1) & & & & & & & & & & & & & & \\
\hline \multirow[t]{3}{*}{66} & & Tapirus terrestris (Linnaeus, 1758) & Tapir, sachavaca & 1 & 1 & 1 & 1 & 1 & 1 & 1 & 1 & $\begin{array}{l}{[246,248,256][114,144][55,87,205,207,208,} \\
212,216,222,232][54,71,99,173][3,10,12,20, \\
38,67,104,159,169][37,50,52,53,56,62,76] \\
{[221,230,233,234,239,241,245,255,261,290,} \\
291,292][263,269,287,289]\end{array}$ & 50 & & VU & NT \\
\hline & \multicolumn{2}{|c|}{ Artiodactyla (6) } & & & & & & & & & & & & & & \\
\hline & \multicolumn{2}{|r|}{ Tayassuidae (2) } & & & & & & & & & & & & & & \\
\hline 67 & & Dicotyles tajacu (Linnaeus, 1758) & Sajino, pecarí de collar & & 1 & 1 & 1 & 1 & 1 & 1 & 1 & $\begin{array}{l}{[129,139,144][48,55,63,87,89,217,225,228,} \\
232][54,70,71,77,99,173,177,181,183][1,3, \\
10,12,20,21,24,38,67,75,94,104,126,154 \\
159,169,171,182][2,7,8,37,43,50,52,53,56, \\
62,76][221,230,233,234,235,238,239,241 \\
242,243,245,255,261,290,291][263,264,269 \\
285,287,289]\end{array}$ & $71^{* *}$ & & LC & \\
\hline 68 & & Tayassu pecari (Link, 1795) & $\begin{array}{l}\text { Huangana, pecarí labiado, pecarí bo- } \\
\text { quiblanco }\end{array}$ & 1 & 1 & 1 & 1 & 1 & 1 & 1 & 1 & $\begin{array}{l}{[256][139][48,55,63,170,207,208,212,216,} \\
222][54,71,173,181][3,10,12,20,21,24,38, \\
67,75,100,122,159,169,171,182,205][2,8,37, \\
50,52,53,56,62,76][221,230,233,234,239, \\
241,242,243,245,255,291][268,285,287,289]\end{array}$ & 55 & & Vu & NT \\
\hline
\end{tabular}

REVISTA PERUANA DE BIOLOGÍA 28(ESPECIAL): E21910 (DICIEMBRE 2021) 
DIAZ ET AL.

\begin{tabular}{|c|c|c|c|c|c|c|c|c|c|c|c|c|c|c|c|}
\hline & Nombre científico & Nombre común & $\frac{\xi}{\&}$ & $\sum_{\square}$ & ذَ & 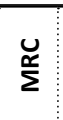 & $\sum_{\Sigma}^{\frac{\pi}{\pi}}$ & $\vec{z}$ & ष্ׁ & ऽூ & Localidades & $\mathbf{z}$ & 몸 & $\underline{\underline{z}}$ & 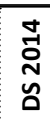 \\
\hline & \multicolumn{15}{|l|}{ Cervidae (2) } \\
\hline 69 & Mazama americana (Erxleben, 1777) & Venado colorado, venado rojo & 1 & 1 & 1 & 1 & 1 & 1 & 1 & 1 & $\begin{array}{l}{[246,248,256][144][55,87,172,225,228,232]} \\
{[54,71,99,173][3,10,12,20,38,67,104,109,} \\
122,159,169][37,50,52,53,56,62,76][221 \\
230,233,234,239,241,261,291,292][267,284 \\
287,289]\end{array}$ & 45 & & $\mathrm{DD}$ & $\mathrm{DD}$ \\
\hline \multirow[t]{2}{*}{70} & Mazama nemorivaga (F. Cuvier, 1817) & $\begin{array}{l}\text { Venado cenizo, venado gris, venado } \\
\text { pardo }\end{array}$ & 1 & 1 & 1 & 1 & 1 & 1 & 1 & 1 & $\begin{array}{l}{[246][114][49,55,87,211][54,71,173][3,10,} \\
12,20,104,109,142,159,169][37,50,52,76] \\
{[234,239,241,255,261][277]}\end{array}$ & 28 & & LC & \\
\hline & \multicolumn{15}{|l|}{ Delphinidae (1) } \\
\hline \multirow[t]{2}{*}{71} & Sotalia fluviatilis (Gervais \& Deville, 1853) & Bufeo gris, delfín gris & & & 1 & 1 & & 1 & & & $\begin{array}{l}{[188,194,196,199,200,202,203,204,212,251]} \\
{[54][56,62]}\end{array}$ & 13 & & $\mathrm{DD}$ & $\mathrm{DD}$ \\
\hline & \multicolumn{15}{|l|}{ Iniidae (1) } \\
\hline \multirow[t]{2}{*}{72} & Inia geoffrensis (Blainville, 1817) & Bufeo colorado, delfín rosado & & & 1 & 1 & 1 & 1 & 1 & & $\begin{array}{l}{[164,212,232][54][105,120,121,122,140][37,} \\
56,62][261]\end{array}$ & 13 & & EN & $\mathrm{DD}$ \\
\hline & Total especies (72) & & 23 & 24 & 54 & 46 & 66 & 44 & 57 & 41 & & & 5 & 72 & 13 \\
\hline
\end{tabular}




\section{Literatura citada}

Acosta LE, Garbino GST, Gasparini GM, Parisi-Dutra R. 2020. Unraveling the nomenclatural puzzle of the collared and white-lipped peccaries (Mammalia, Cetartiodactyla, Tayassuidae). Zootaxa 4851 (1): 060-080. https://dx.doi. org/10.11646/zootaxa.4851.1.2

Allgas N, Shanee S, Shanee N, de Palomino HC. 2018. Rapid Survey of the Primate Density and Biomass at Katakari, Pacaya Samiria National Reserve, Peru. Primate Conservation 32: $57-66$.

Amanzo J. 2006. Mamíferos medianos y grandes. Pp. 98-106. En: C Vriesendorp, N Pitman, JI Rojas, et al., eds. Perú: Matsés. Rapid Biological Inventories Report 16. Chicago, Illinois, The Field Museum. 131 pp.

Anacleto TCS, Perez-Godoy L, Pukenis-Tubelis D. 2013. New records of the southern naked-tailed armadillo Cabassous unicinctus unicinctus Linnaeus, 1758 (Cingulata: Dasypodidae) in Brazil. Biota Neotropical 13(2): 233-296. https://dx.doi.org/10.1590/S167606032013000200028

Aquino R. 2005. Alimentación de mamíferos de caza en los «aguajales» de la Reserva Nacional de Pacaya-Samiria (Iquitos, Perú). Revista Peruana de Biología 12(3): 417-425. https://doi.org/10.15381/rpb.v12i3.2416

Aquino R, Alvarez J, Mulanovich A. 2005a. Diversidad y estado de conservación de primates en las Sierras de Contamana, Amazonia peruana. Revista Peruana de Biología 12(3): 427-434. https://doi.org/10.15381/rpb.v12i3.2417

Aquino R, Ique C, Gálvez H. 2005b. Reconocimiento preliminar de la densidad y estructura poblacional de Saguinus tripartitus Milne-Eduards en la Amazonia peruana. Revista Peruana de Biología 12(3): 435-440. https://doi. org/10.15381/rpb.v12i3.2418

Aquino, R, Terrones C, Navarro R, Terrones W. 2007a. Evaluación del impacto de la caza en mamíferos de la cuenca del río Alto Itaya, Amazonia peruana. Revista Peruana de Biología 14(2): 181-186. https://dx.doi.org/10.15381/ rpb.v14i2.1725

Aquino R, Pacheco T, Vásquez M. 2007. Evaluación y valorización económica de la fauna silvestre en el río Algodón, Amazonia peruana. Revista peruana de Biología 14(2): 187192. https://doi.org/10.15381/rpb.v14i2.1730

Aquino R, Terrones W, Cornejo F, Heymann EW. 2008. Geographic distribution and possible taxonomic distinction of Callicebus torquatus populations (Pitheciidae: Primates) in Peruvian Amazonia. American Journal of Primatology 70(12): 1181-1186. https://dx.doi.org/10.1002/ ajp.20607

Aquino R, Terrones W, Navarro R, Terrones C, Cornejo FM. 2009a. Caza y estado de conservación de primates en la cuenca del río Itaya, Loreto, Perú. Revista Peruana de Biología 15(2): 33-40. https://doi.org/10.15381/rpb.v15i2.1719

Aquino R, Gil D, Pezo E. 2009b. Aspectos ecológicos y sostenibilidad de la caza del majás (Cuniculus paca) en la cuenca del río Itaya, Amazonia peruana. Revista Peruana de Biología 16(1): 67-72. https://doi.org/10.15381/rpb. v16i1.179

Aquino R, Meléndez G, Pezo E, Gil D. 2012a. Tipos y formas de ambientes de dormir de majás (Cuniculus paca) en la cuenca alta del río Itaya. Revista Peruana de Biología 19(1): 27-34. https://doi.org/10.15381/rpb.v19i1.800

Aquino R, Tuesta C, Rengifo E. 2012b. Diversidad de mamíferos y sus preferencias por los tipos de hábitats en la cuenca del río Alto Itaya, Amazonia peruana. Revista Peruana de Biología 19(1): 35-42. https://doi.org/10.15381/rpb.

\section{v19i1.785}

Aquino R, Cornejo FM, Pezo E, Heymann EW. 2013. Distribution and abundance of white-fronted spider monkeys, Ateles belzebuth (Atelidae), and threats to their survival in Peruvian Amazonia. Folia Primatologica 84(1): 1-10. https://doi.org/10.1159/000345549

Aquino R, López L, García G, Heymann EW. 2014a. Diversity, abundance and habitats of the primates in the río Curaray basin, Peruvian Amazonia. Primate Conservation (28): 1-8. https://doi.org/10.1896/052.028.0103

Aquino R, Charpentier E, García G. 2014. Diversidad y abundancia de primates en hábitats del área de influencia de la carretera Iquitos-Nauta, Amazonia Peruana. Ciencia Amazónica (Iquitos) 4(1): 3-12. https://dx.doi. org/10.22386/ca.v4i1.62

Aquino R, López L, Arévalo I, García G, Charpentier E. 2014c. Densidad de ungulados en bosques de baja y alta presión de caza en el nororiente de la Amazonia peruana. Ciencia Amazónica (Iquitos) 4(2): 128-137.

Aquino R, López L, García G, Arévalo I, Charpentier E. 2015. Situación actual de primates en bosques de alta perturbación del nororiente de la Amazonia peruana. Ciencia Amazónica (Iquitos) 5(1): 50-60.

Aquino R, López L, Arévalo I, Daza J. 2016. Diversidad y abundancia de primates y sus amenazas en el interfluvio de los ríos Napo y Putumayo, Amazonia peruana. Revista peruana de biología 23(3): 243-252. http://dx.doi. org/10.15381/rpb.v23i3.12859

Aquino R, Bodmer RE. 2004. Plantas útiles en la alimentación de Primates en la cuenca del Río Samiria, Amazonia Peruana. Neotropical Primates 12(1): 1-6.

Aquino R, Bodmer RE. 2006. Distribución y abundancia de Ateles belzebuth E. Geoffroy y Ateles chamek Humboldt (Cebidae: Primates) en la reserva nacional Pacaya Samiria, Perú. Revista Peruana de Biología 13(1): 103-106. https://doi.org/10.15381/rpb.v13i1.1769

Aquino R, Calle A. 2003. Evaluación del estado de conservación de los mamíferos de caza: Un modelo comparativo en comunidades de la Reserva Nacional Pacaya Samiria (Loreto, Perú). Revista peruana de biología 10(2): 163-174. https://doi.org/10.15381/rpb.v10i2.2498

Aquino R, Encarnación F. 1986. Characteristics and use of sleeping sites in Aotus (Cebidae: Primates) in the Amazon lowlands of Peru. American Journal of Primatology 11(4): 319-331.

Aquino R, Encarnacion F. 1996. Distribución geográfica de Saguinus tripartitus en la Amazonia del Perú. Neotroprical Primates 4(1): 1-4.

Aquino R, Puertas P. 1996. Observaciones preliminares sobre la ecología de Speothos venaticus (Canidae: Carnivora) en su hábitat natural. Folia Amazónica 8(1): 131-143. https://dx.doi.org/10.24841/fa.v8i1.309

Bardales-Alvites C, Torres-Oyarce L, Tirado-Herrera E, Antúnez-Correa M. 2017. Presión de caza en mamíferos de la Reserva Nacional Matsés, al noreste de la Amazonia Peruana. Folia Amazónica 26(1): 75-84. https://doi. org/10.24841/fa.v26i1.420

Bennett CL, Leonard S, Carter S. 2001. Abundance, diversity, and patterns of distribution of primates on the Tapiche River in Amazonian Peru. American Journal of Primatology 54(2): 119-126.

Bowler MT, Bodmer RE. 2011. Diet and food choice in Peruvian red uakaris (Cacajao calvus ucayalii): selective or opportunistic seed predation?. International Journal of Prima- 
tology: 32(5): 1109-1122.

Bowler MT, Tobler MW, Endress BA, Gilmore MP, Anderson MJ. 2017. Estimating mammalian species richness and occupancy in tropical forest canopies with arboreal camera traps. Remote Sensing in Ecology and Conservation 3(3): 146-157.

Bravo AD, Lizcano J, Álvarez-Loayza P. 2016. Mamíferos medianos y grandes/Large and medium-sized mammals. Pp. 140-151, 320-329, 494-497. En: N Pitman, A Bravo, S Claramunt, et al., eds. Perú: Medio Putumayo-Algodón. Rapid Biological and Social Inventories Report 28. The Field Museum, Chicago. 519 pp.

Britto B. 2017. Actualización de las ecorregiones terrestres de Perú propuestas en el Libro Rojo de Plantas Endémicas del Perú. Gayana. Botánica 74(1): 15-29. http://dx.doi. org/10.4067/S0717-66432017005000318

Charpentier EJ, García G, Aquino R. 2015. Uso y competición por plantas alimenticias entre Pithecia aequatorialis (Primates: Pitheciidae) y otros animales en la Amazonia peruana. Revista Peruana de Biología 22(2): 225-232. https:// doi.org/10.15381/rpb.v22i2.11356

Coimbra RTF, Miranda FR, Lara CC, et al. 2017. Phylogeographic history of South American populations of the silky anteater Cyclopes didactylus (Pilosa: Cyclopedidae). Genetics and Molecular Biology 40(1): 40-49. https://dx.doi. org/10.1590/1678-4685-gmb-2016-0040

Conga DF, Bowler M, Tantalean M, et al. 2014. Intestinal helminths in wild Peruvian red uakari monkeys (Cacajao calvus ucayalii) in the northeastern Peruvian Amazon. Journal of Medical Primatology 43(2): 130-133. https://dx.doi. org/10.1111/jmp.12092

Conga DF, Giese EG, Serra-Freire NM et al. 2016. Morphology of the oxyurid nematodes Trypanoxyuris (T.) cacajao n. sp. and T.(T.) ucayalii n. sp. from the red uakari monkey Cacajao calvus ucayalii in the Peruvian Amazon. Journal of Helminthology 90(4): 483-493. https://doi.org/10.1017/ S0022149X1500067X

Conga DF, Mayor P, Furtado AP, et al. 2018. Co-infection with filarial nematodes in Sapajus macrocephalus and Cebus albifrons (Primates: Cebidae) from the Peruvian Amazon. Journal of helminthology, 1-4. https://dx.doi. org/10.1017/S0022149X18000287

DS $N^{\circ}$ 004-2014-AG. 2014. Decreto Supremo que aprueba la actualización de la lista de clasificación y categorización de las especies amenazadas de fauna silvestre legalmente protegidas, Ministerio de Agricultura y Riego. 8 de abril del 2014. El Peruano Normas Legales: 520497-520504.

Emmons LH, Feer F. 1997. Neotropical rainforest mammals: a field guide. 2nd edition. University of Chicago Press. 307 pp.

Falcón-Ayapi RH, Hidalgo-Vilchez MD, Gonzales-Tanchiva CN, et al. 2019. Fauna registrada por trampas cámara en la Reserva Nacional Pucacuro. Field Guide 1123: 1-3. <https:// fieldguides.fieldmuseum.org/guides/guide/1123>. Acceso 14/09/20.

Fleck DW, Voss RS, Patton JL. 1999. Biological basis of saki (Pithecia) folk species recognized by the Matses Indians of Amazonian Perú. International Journal of Primatology 20(6): 1005-1028.

Fleck DW, Harder JD. 2000. Matses Indian rainforest habitat classification and mammalian diversity in Amazonian Peru. Journal of Ethnobiology 20(1): 1-36.

Garbino GS, Casali DM, Nascimento FO, et al. 2019. Taxonomy of the pygmy marmoset (Cebuella Gray, 1866): Geographic variation, species delimitation, and nomenclatural notes. Mammalian Biology 95(2019): 135-142. https://dx.doi. org/10.1016/j.mambio.2018.09.003

Gardner AL, ed. Mammals of South America, Volume 1. Marsupials, Xenarthrans, Shrews, and Bats. The University of Chicago Press, Chicago and London. 669 pp.

Gilmore MP. 2005. An ethnoecological and ethnobotanical study of the Maijuna Indians of the Peruvian Amazon. Doctoral dissertation, Miami University.

Glander KE, Tapia RJ, FachinTA. 1984. The impact of cropping on wild populations of Saguinus mystax and Saguinus fuscicollis in Peru. American Journal of Primatology 7(2): 89-97.

Griffiths B, Gilmore M, Bowler M. 2020. Predation of a Brazilian porcupine (Coendou prehensilis) by an ocelot (Leopardus pardalis) at a mineral lick in the Peruvian Amazon. Food Webs: e00148. https://dx.doi:10.1016/j. fooweb.2020.e00148

Hershkovitz P. 1977. Living New World Monkeys (Platyrrhini): With an Introduction to Primates, Volume 1. University of Chicago Press, Chicago. 1132 pp.

Hershkovitz P. 1983. Two new species of night monkeys, genus Aotus (Cebidae, Platyrrhini): a preliminary report on Aotus taxonomy. American Journal of Primatology 4(3): 209-243. https://dx.doi.org/10.1002/ajp.1350040302

Hershkovitz P. 1984. Taxonomy of Squirrel Monkey genus Saimiri (Cebidae, Platyrrhini): A Preliminary report with Description of Hitherto Unnamed Form. American Journal of Primatology 7: 155-210. https://dx.doi.org/10.1002/ ajp.1350070212

Hershkovitz P. 1987. The taxonomy of South American sakis, genus Pithecia (Cebidae, Platyrrhini): A preliminary report and critical review with the description of a new species and a new subspecies. American Journal of Primatology 12(4): 387-468. https://dx.doi.org/10.1002/ ajp.1350120402

Hershkovitz P. 1990. Titis, New World monkeys of the genus Callicebus (Cebidae, Platyrrhini): A Preliminary Taxonomic Review. Fieldiana Zoology 5: 1-109.

Heymann EW. 1990a. Interspecific relations in a mixed-species troop of moustached tamarins, Saguinus mystax, and saddle-back tamarins, Saguinus fuscicollis (Platyrrhini: Callitrichidae), at the Rio Blanco, Peruvian Amazonia. American Journal of Primatology 21(2): 115-127. https://dx.doi.org/10.1002/ajp.1350210205

Heymann, EW. 1990b. Reactions of wild tamarins, Saguinus mystax andSaguinus fuscicollis to avian predators. International Journal of Primatology 11(4): 327-337. https:// dx.doi.org/10.1007/BF02193004

Heymann EW. 1995. Sleeping habits of tamarins, Saguinus mystax and Saguinus fuscicollis (Mammalia; Primates; Callitrichidae), in north-eastern Peru. Journal of Zoology 237(2): 211-226. https://doi.org/10.1111/j.1469-7998.1995. tb02759.x

Heymann EW, Encarnación F, Canaquin JE. 2002. Primates of the Rio Curaray, northern peruvian Amazon. International Journal of Primatology 23(1): 191-201.

Heymann EW, Amasifuén CF, Tello NS, et al. Disgusting appetite: Two-toed sloths feeding in human latrines. Mammalian Biology 76: 84-86. https://doi.org/10.1016/j.mambio.2010.03.003

Heymann EW, Aquino R. 1994. Exploraciones primatológicas en las quebradas Blanco, Blanquillo y Tangara, Amazonpia Peruana. Folia Amazónica 6(1-2): 135-149. https:// dx.doi.org/10.24841/fa.v6i1-2.252

Hice CL, Velazco PM. 2012. The Non-volant Mammals of the Reser- 
va Nacional Allpahuayo-Mishana, Loreto, Peru. Special Publications of the Museum of Texas Tech University 60 : 1-135.

Jorge ML, Velazo P. 2006. Mamíferos grandes. Pp. 98-106. En: C Vriesendorp, TS Schulenberg, WS Alverson, DK Moskovits y JI Rojas-Moscoso, eds. Perú: Sierra del Divisor. Rapid Biological and Social Inventories Report 17. The Field Museum, Chicago. 120 pp.

Kolowski J, Alonso A. 2010. Density and activity patterns of ocelots (Leopardus pardalis) in northern Peru and the impact of oil exploration activities. Biological Conservation 143: 917-925. https://dx.doi.org/10.1016/j.biocon.2009.12.039

Lonnberg E. 1921. A second contribution to the mammalogy of Ecuador with some remarks on Caenolestes. Arkiv för zoologi 14(4): 1-104.

Marsh LK. 2014. A Taxonomic Revision of the Saki Monkeys, Pithecia Desmarest, 1804. Neotropical Primates 21(1): 1-165. https://dx.doi.org/10.1896/044.021.0101

Matauschek C. 2010. Taxonomy, phylogeny and distribution of tamarins (genus Saguinus, Hoffannsegg 1807). Tesis, Grado de Doctor. Gottingen, Alemania.

Mayor P, Pérez-Peña P, Bowler M, et al. 2015. Effects of selective logging on large mammal populations in a remote indigenous territory in the northern Peruvian Amazon. Ecology and Society 20(4): 1-10.

Mayor P, Bizri E, Bodmer RE, Bowler M. 2017. Assessment of mammal reproduction for hunting sustainability through community-based sampling of species in the wild. Conservation Biology 31(4): 912-923. https://dx.doi. org/10.1111/cobi.12870

Mayor P, Bowler M. 2015. Low birthrates and high levels of female reproductive inactivity may characterize the reproductive biology of wild Peruvian red uakaris (Cacajao calvus ucayalii). Journal of medical primatology: 44(1): 27-34. https://dx.doi.org/10.1111/jmp.12155

McAfee RK. 2014. Dental anomalies within extant members of the mammalian Order Pilosa. Acta Zoologica 96(3): 301311. https://dx.doi.org/10.1111/azo.12077

McGuire TL. 2010. Ecology and conservation status of tucuxi (Sotalia fluviatilis) in the Pacaya-Samiria Reserve, Peru. Latin American Journal of Aquatic Mammals, 8(1-2): 103110.

McGuire TL, Aliaga-Rossel ER. 2007. Seasonality of Reproduction in Amazon River Dolphins (Inia geoffrensis) in Three Major River Basins of South America. Biotropica 39(1): 129-135. https://dx.doi.org/10.1111/j.17447429.2006.00221.x

Montenegro 0, Moya L. 2011. Mamíferos. Pp. 126-133. En: Pitman, N., C. vriesendorp, D. K. Moskovits, R. von May, D. Alvira, T. Wachter, D.F. Stotz, y/and á. del Campo, eds. 2011. Perú: Yaguas-Cotuhé. Rapid Biological and Social Inventories Report 23. The Field Museum, Chicago. 154 pp.

Nadjafzadeh MN, \& Heymann EW. 2008. Prey foraging of red titi monkeys, Callicebus cupreus, in comparison to sympatric tamarins, Saguinus mystax and Saguinus fuscicollis. American Journal of Physical Anthropology 135(1): 5663.

Navarro R, Terrones C. 2006. Evaluación del impacto de la caza en mamíferos de la cuenca alta del río Itaya, Loreto-Perú. Tesis, Biólogo. Universidad Nacional de la Amazonia Peruana. $104 \mathrm{pp}$.

Nickle DA, Heymann EW. 1996. Predation on Orthoptera and other orders in insects by tamarin monkeys, Sagui- nus mystax mystax and Saguinus fuscicollis nigrifrons (Primates: Callitrichidae), in north-eastern Peru. Journal of Zoology 239(4): 799-819. https://doi org/10.1111/j.1469-7998.1996.tb05479.x

Osgood WH. 1914. Mammals of an expedition across northern Peru. Field Museum of Natural History, Zoological Series 10: $143-185$.

Pacheco V. 2002. Mamíferos del Perú. Diversidad y conservación de los mamíferos neotropicales. Conabio-UNAM. México, DF. Pp. 503-550.

Pacheco V, Arias L. 2001. Mammals. Pp. 155-158. In: LO Rodriguez and DK Moskovitz, eds. Perú: Biabo Cordillera Azul. Rapid Biological Inventories Report 2. Chicago, IL: The Field Museum. <https://www.academia.edu/731052/ Rapid_Biological_Inventories_Per\%C3\%BA_Biabo_Cordillera_Azul>. Acceso 28/10/2020."

Pacheco V, Pezo R. 1982. Evaluacion de la Fauna Silvestre en la Estación Biológica Pithecia. En: Investigación y Utilización Racional de la Fauna Silvestre del Bosque Tropical Humedo. MAB-Perú, Lima.

Pacheco V, Cadenillas R, Salas E, et al. 2009. Diversidad y endemismo de los mamíferos del Perú. Revista Peruana de Biología 16(1): 5-32. https://doi.org/10.15381/rpb. v16i1.111

Pacheco V, Graham-Angeles L, Diaz S, et al. 2020. Diversidad y distribución de los mamíferos del Perú por departamentos y ecorregiones I. Revista Peruana de Biología 27 (3): 289328. https://dx.doi.org/10.15381/rpb.v27i3.18356

Pacheco V, Diaz S, Graham-Angeles L, et al. 2021. Lista actualizada de la diversidad de los mamíferos del Perú y una propuesta para su actualización. Revista Peruana de Biología 28(4): e21019. https://dx.doi.org/10.15381/rpb. v28i4.21019

Patterson BD, López-Wong C. 2014. Mamíferos/Mammals. Pp. 154-167. In: N Pitman, C Vriesendorp, D Alvira, et al., eds. Perú: Cordillera Escalera-Loreto. Rapid Biological and Social Inventories Report 26. The Field Museum, Chicago. $541 \mathrm{pp}$.

Patton JL. 2015. Familia Dinomydae. Pp. 783-785. In: JL Patton, UF Pardiñas and G D’Elía, eds. 2015. Mammals of South America, volume 2: rodents. University of Chicago Press. $1363 \mathrm{pp}$.

Patton JL, Pardiñas U, D’Elía G, eds. 2015. Mammals of South America, volume 2: Rodents. University of Chicago Press. xxvi $+1336 \mathrm{pp}$.

Pérez-Peña PE, Ruck L, Riveros M, Rojas G. 2012. Evaluación del conocimiento indígena Kichwa como herramienta de monitoreo en la abundancia de animales de caza. Folia Amazonica 21(1-2): 115-128.

Pérez-Peña PE, Aguinda S, Riveros MS, et al. 2016. Distribución y abundancia del supay pichico Callimico goeldii (Thomas, 1904) en la Reserva Nacional Pucacuro, al norte de la Amazonia peruana. Folia Amazónica 25(2): 167-177.

Pérez-Peña P, Riveros MS, Mayor P, et al. 2017. Estado poblacional del sajino Pecari tajacu y huangana Tayassu pecari en la Amazonia peruana. Folia Amazónica 26(2): 103-120.

Pérez-Peña PE, Bardales-Alvites C, Ramos-Rodríguez MC, et al. 2019. Mamíferos. Pp. 128-151. En: PE Pérez-Peña, MC Ramos-Rodriguez, J Díaz, et al., eds.Biodiversidad en las cuencas del Napo y Curaray, Perú. Instituto de Investigaciones de la Amazonia peruana. Impreta Luanos EIRL. $203 \mathrm{pp}$.

Pitman N, Gagliardi-Urrutia G, Jenkins C. 2013. La Biodiversidad de Loreto, Perú: El conocimiento actual de la diversidad 
de plantas y vertebrados terrestres. Center for International Environmental Law, Washington, DC, USA. 40 pp.

Pittet M, Bennett P. 2014. Examining the state of biodiversity using camera traps in the Pacaya Samiria National Reserve, Peru. Pp. 53-60. In: P Meek, D Fleming, P Ballard, et al., eds. Camera trapping: wildlife management and research (Vol. 2). Melbourne, Australia: Csiro Publishing. $392 \mathrm{pp}$.

Puertas PE, Aquino R, Encarnación F. 1992. Uso de alimentos y competición entre el mono nocturno Aotus vociferans y otros mamíferos, Loreto, Perú. Folia Amazónica 4(2): 147-156. https://dx.doi.org/10.24841/fa.v4i2.213

Puertas P, Bodmer R, Aquino R. 1995. Diversidad y conservación de primates en la reserva comunal Tamshiyacu-Tahuayo, Loreto, Perú. Folia Amazonica 7(1-2): 113-127.

Puertas P, Pinedo A, Soplin S, et al. 2017. Evaluación poblacional y uso sostenible de animales de caza por comunidades indígenas en el área de conservación regional Ampiyacu Apayacu, noreste de la Amazonia peruana. Folia Amazónica 26(1): 37-50. https://dx.doi.org/10.24841/ fa.v26i1.417

Quintana H, Pacheco V, Salas E. 2009. Diversidad y conservación de los mamíferos de Ucayali, Perú. Ecología Aplicada 8(12): 91-103.

Rylands AB, Matauschek C, Aquino R, et al. 2011. The range of the golden-mantle tamarin, Saguinus tripartitus (Milne Edwards, 1878): distributions and sympatry of four tamarin species in Colombia, Ecuador, and northern Peru. Primates 52(1): 25-39.

Saldaña RJS, Saldaña HVL. 2011. La cacería de animales silvestres en la comunidad de bretaña, río puinahua, loreto - perú. Revista Colombiana de Ciencia Animal-RECIA 3(2): 225237. https://dx.doi.org/10.24188/recia.v3.n2.2011.369

Satizábal P, Mignucci-Giannoni AA, Duchêne S, et al. 2012. Phylogeography and Sex-Biased Dispersal across Riverine Manatee Populations (Trichechus inunguis and Trichechus manatus) in South America. PLoS one 7(12): e52468. https://dx.doi.org/10.1371/journal.pone.0052468

Sittig L, Römer U, Gilleman C, Gongora-Torres NG. 2018. Population numbers of Amazon river dolphins Inia geoffrensis and Sotalia fluviatilis (Mammalia: Cetaceae: Delphinidae) in the lower Río Tigre region, Loreto, Perú. Vertebrate Zoology 68: 213-224.

Soini P. 1982. Ecology and population dynamics of the pygmy marmoset, Cebuella pygmaea. Folia Primatologica 39(1-2): 1-21. https://dx.doi.org/10.1159/000156066

Soini P. 1986. A synecological study of a primate community in the Pacaya-Samiria National Reserve, Peru. Primate Conservation 7: 63-71.

Soini P. 1987. Ecology of the saddle-back tamarin Saguinus fuscicollis illigeri on the Rio Pacaya, northeastern Peru. Folia Primatologica 49(1): 11-32. https://dx.doi. org/10.1159/000156305

Soini P. 1992. Ecología del coto mono (Alouatta seniculus, Cebidae) en el río Pacaya, Reserva nacional Pacaya-Samiria Perú. Folia Amazonica 4(2): 117-134.

Stephens L, Traylor MA. 1983. Ornithological gazetteer of Peru. Museum of Comparative Zoology, Harvard University. $271 \mathrm{pp}$.

Tapia-Ruiz J, Gálvez-Carrillo H. 2012. Estructura poblacional de Callicebus cupreus (Primates: Cebidae) en la Amazonia peruana. Ciencia Amazónica (Iquitos) 2(2): 93-99. https://dx.doi.org/10.22386/ca.v2i2.31

Terrones-Ruíz WI, Vela-Diaz DM, Flores-Amasifuén C, Heymann
EW. 2004. Diurnal birth of a wild Red Titi Monkey Callicebus cupreus, at the Estación Biológica Quebrada Blanco. Neotropical Primates 12(1): 15-16.

Teta P. 2019. Geographic variation in quantitative skull traits in the genus Myoprocta Thomas, 1903 (Rodentia, Dasyproctidae) and its taxonomic implications. Mammalia 83(3): 212-218.

Thomas 0. 1928. The Godman-Thomas Expedition to Peru. VII. The mammals of the Rio Ucayali. Annals and Magazine of Natural History 2(9): 249-265. https://dx.doi. org/10.1080/00222932808672875

Torres-Oyarce L, Bardales-Alvites C, Tirado-Herrera E, AntúnezCorrea M. 2017. Estado de las poblaciones de mamíferos en la Reserva Nacional Matsés, al noreste de la Amazonia Peruana. Folia Amazónica 26(2): 153-160. https:// dx.doi.org/10.24841/fa.v26i2.428

Vermeer J, Tello-Alvarado JC, Villacis del Castillo JT, Bóveda-Penalba AJ. 2013. A New Population of Red Uakaris (Cacajao Calvus ssp.) in the Mountains of North-Eastern Peru. Neotropical Primates 20(1): 12-17. https://dx.doi. org/10.1896/044.020.0103

Voss RS. 2011. Revisionary notes on Neotropical porcupines (Rodentia: Erethizontidae) 3. An annotated checklist of the species of Coendou Lacépède, 1799. American Museum Novitates (3720): 1-36.

Voss RS, Emmons LH. 1996. Mammalian Diversity in Neotropical Lowland Rainforests: a Preliminary Assessment. Bulletin of the American Museum of Natural History 230: 1-115. http://hdl.handle.net/2246/1671

Voss RS, Fleck DW. 2011. Mammalian Diversity and Matses Ethnomammalogy in Amazonian Peru Part 1: Primates. Bulletin of the American Museum of Natural History Number 351: 1-81. https://dx.doi.org/10.1206/351.1

Voss RS, Fleck DW. 2017. Mammalian Diversity and Matses Ethnomammalogy, in Amazonian Peru, Part 2: Xenartha, Carnivora, Perisodactyla, Artiodactyla and Sirenia. Bulletin of the American Museum of Natural History 417: 1-118. https://dx.doi.org/10.1206/00030090-417.1.1

\begin{abstract}
Agradecimientos / Acknowledgments:
A los miembros del departamento de Mastozoología del Museo de Historia Natural UNMSM por el apoyo en la identificación de los especímenes. Un agradecimiento especial a José SerranoVillavicencio y Javier Barrio por la revisión y sugerencias de los mapas preliminares.
\end{abstract}

\section{Conflicto de intereses / Competing interests:}

VP es editor de la RPB, no participó en el el proceso de edición. Los autores no incurren en conflictos de intereses.

\section{Rol de los autores / Authors Roles:}

SD: conceptualización, Investigación, Análisis formal, EscrituraPreparación del borrador original, Redacción: revisión y edición. PS-V, LG-A, VP: Investigación, Análisis formal, Redacción: revisión y edición.

\section{Fuentes de financiamiento / Funding:}

Este trabajo se realizó con financiamiento de Biosfera Consultores Ambientales S.A.C. (BIOCAM-504-2020).

\section{Aspectos éticos / legales; Ethics / legals:}

Los autores declaran no haber incurrido en ningún aspecto antiético ni omitido norma legal nacional o internacional en la investigación y elaboración del articulo. 
Anexo 1 Lista de localidades, coordenadas y fuente incluidas en los análisis.

\begin{tabular}{|c|c|c|c|}
\hline Pto & Localidad & Longitud & Latitud \\
\hline 1 & Maynas; Torres Causana; Lagartococha River mouth & -75.261389 & -0.655000 \\
\hline 2 & Putumayo; Teniente Manuel Clavero; Río Angusilla & -74.382738 & -0.837857 \\
\hline 3 & Maynas; Torres Causana; Aguarico bajo & -75.212333 & -0.860306 \\
\hline 4 & Maynas; Torres Causana; Pantoja, Río Napo, left bank & -75.182444 & -0.960917 \\
\hline 5 & Maynas; Torres Causana; Río Napo, Aguarico & -75.199056 & -0.986250 \\
\hline 6 & Maynas; Torres Causana; Santa María & -75.000000 & -1.000000 \\
\hline 7 & Putumayo; Teniente Manuel Clavero; Río Yubineto & -74.337390 & -1.005805 \\
\hline 8 & Putumayo; Teniente Manuel Clavero; Río Putumayo, cuenca alta & -74.172591 & -1.024428 \\
\hline 9 & Maynas; Torres Causana; Vencedores, Río Napo, right bank & -75.033333 & -1.200000 \\
\hline 10 & Maynas; Napo; Nashiño medio & -75.266000 & -1.259889 \\
\hline 11 & Maynas; Torres Causana; Tempestad, south bank of Río Napo & -74.888528 & -1.282528 \\
\hline 12 & Maynas; Napo; Nashiño bajo & -75.245111 & -1.441694 \\
\hline 13 & Maynas; Napo; Puerto Arica, north bank of Río Curaray & -75.200000 & -1.466667 \\
\hline 14 & Maynas; Napo; Nashiño & -75.384112 & -1.493584 \\
\hline 15 & Maynas ; Napo; Colpa & -75.430801 & -1.566575 \\
\hline 16 & Maynas; Torres Causana; Río San José & -74.883528 & -1.567194 \\
\hline 17 & Maynas; Torres Causana; Río San Antonio & -74.910098 & -1.583274 \\
\hline 18 & Maynas; Napo; Block 39 of an oil concession & -75.205556 & -1.586750 \\
\hline 19 & Maynas ; Napo; Shuyal & -75.227013 & -1.611599 \\
\hline 20 & Maynas ; Napo; Curaray & -75.397989 & -1.618666 \\
\hline 21 & Maynas; Napo; Río Curaray & -75.417801 & -1.696321 \\
\hline 22 & Maynas; Torres Causana; Río Aushiri & -74.820185 & -1.718976 \\
\hline 23 & Maynas; Napo; Río Arabela & -75.480357 & -1.733851 \\
\hline 24 & Maynas; Napo; Tamboryacu & -73.959039 & -1.900066 \\
\hline 25 & Maynas; Napo; Campo Serio, Rio Napo, right bank & -74.931472 & -1.982194 \\
\hline 26 & Maynas; Napo; Bellavista, Río Yuvineto, south of Río Putumayo & -74.550000 & -2.033333 \\
\hline 27 & Maynas; Napo; Puerto Elvira, south bank of Río Napo & -74.533333 & -2.033333 \\
\hline 28 & Maynas; Napo; Playa, Río Curaray & -74.900000 & -2.050000 \\
\hline 29 & Maynas; Napo; Buena Vista & -74.866667 & -2.116667 \\
\hline 30 & Maynas; Napo; Ingano, south bank of Río Napo & -74.183333 & -2.133333 \\
\hline 31 & Maynas; Napo; Casa Calvo & -74.633333 & -2.133333 \\
\hline 32 & Maynas; Napo; Quebrada Arabela & -75.000000 & -2.150000 \\
\hline 33 & Loreto; Tigre; Cerca a Monteverde, Río Tigre, restinga en el banco ligeramente al sur de Monteverde & -74.097500 & -2.185560 \\
\hline 34 & Maynas; Napo; Soledad, Río Curaray, north (left) bank & -74.433333 & -2.266667 \\
\hline 35 & Maynas; Napo; Soledad & -74.466667 & -2.266667 \\
\hline 36 & Maynas; Napo; Soledad, north bank of Río Curaray & -74.416667 & -2.283333 \\
\hline 37 & Putumayo; Yaguas; Quebrada Bufeo & -71.519528 & -2.332333 \\
\hline 38 & Maynas; Napo; Curaray River mouth & -74.090556 & -2.363333 \\
\hline 39 & Maynas; Napo; San Rafael, north bank of Río Curaray, near mouth & -74.110861 & -2.363556 \\
\hline 40 & Maynas; Napo; Near to the river Curaray, El Oriente & -74.083330 & -2.366670 \\
\hline 41 & Maynas; Napo; Desembocadura Río Tambor, Río Napo & -73.650000 & -2.466667 \\
\hline 42 & Putumayo; Putumayo; Alto Algodón & -73.330195 & -2.479988 \\
\hline 43 & Putumayo; Putumayo; Río Napo-Algodón & -73.330195 & -2.479988 \\
\hline 44 & Maynas; Napo; 13 km oeste del rio algodón & -73.371058 & -2.482065 \\
\hline 45 & Putumayo; Putumayo; Lower Río Algodón & -72.083333 & -2.483333 \\
\hline 46 & Maynas; Napo; Santa Clotilde & -73.679139 & -2.489056 \\
\hline 47 & Maynas; Alto Nanay; Quebrada Agua Negra & -74.983510 & -2.493370 \\
\hline 48 & Loreto; Tigre; Río Pucacuro, cuenca alta, Reserva Nacional Pucacuro & -75.183157 & -2.509039 \\
\hline
\end{tabular}




\begin{tabular}{|c|c|c|c|}
\hline Pto & Localidad & Longitud & Latitud \\
\hline 49 & Loreto; Trompeteros; Teniente Lopez & -76.116667 & -2.583333 \\
\hline 50 & Putumayo; Putumayo; Río Algodón & -72.081778 & -2.593778 \\
\hline 51 & Maynas; Napo; Puerta Huamán & -73.400000 & -2.600000 \\
\hline 52 & Putumayo; Putumayo; Medio Algodón & -72.865333 & -2.607889 \\
\hline 53 & Putumayo; Yaguas; Choro & -71.485750 & -2.610611 \\
\hline 54 & Mariscal Ramón Castilla; Pebas; Bajo Algodón & -72.133221 & -2.662617 \\
\hline 55 & Loreto; Tigre; Reserva Nacional Pucacuro & -75.166667 & -2.666667 \\
\hline 56 & Putumayo; Yaguas; Cachimbo & -70.529194 & -2.718306 \\
\hline 57 & Maynas; Napo; Quebrada Huiririma, Rio Napo, right bank & -74.005278 & -2.750000 \\
\hline 58 & Datem del Marañón; Andoas; Capahuari & -76.426716 & -2.791714 \\
\hline 59 & Maynas; Napo; Lower Napo River, north side & -73.524500 & -2.793330 \\
\hline 60 & Maynas; Napo; Sergento Lores, Río Napo, right bank & -74.002778 & -2.800000 \\
\hline 61 & Loreto ; Tigre; Posayo & -75.067563 & -2.826790 \\
\hline 62 & Putumayo; Yaguas; Yaguas & -71.415028 & -2.864861 \\
\hline 63 & Loreto; Tigre; Río Pucacuro, cuenca media, Reserva Nacional Pucacuro & -75.104384 & -2.868345 \\
\hline 64 & Maynas; Napo; Lower Napo River region, E bank Rio Yanayacu, ca. 90 km N Iquitos & -73.247222 & -2.934160 \\
\hline 65 & Loreto ; Tigre; Pañayacu & -75.097673 & -2.980308 \\
\hline 66 & Maynas; Napo; Caserío Puerto Huamán, Río Napo & -73.183333 & -2.983333 \\
\hline 67 & Maynas; Mazan; Sucusari River Basin & -73.000000 & -3.000000 \\
\hline 68 & Maynas; Napo; Río Napo, ca. 1 km E Libertad, 80 km N Iquitos & -73.247222 & -3.024590 \\
\hline 69 & Maynas; Mazan; 1.5 km S Libertad, S bank Rio Napo, 80 km N Iquitos & -73.333330 & -3.046900 \\
\hline 70 & $\begin{array}{l}\text { Mariscal Ramón Castilla; Pebas; Río Yaguasyacu, cuenca alta, Area de Conservación Regional Ampi- } \\
\text { yacu Apayacu }\end{array}$ & -71.898781 & -3.094388 \\
\hline 71 & Mariscal Ramón Castilla; Pebas; Yaguasyacu, Área de Conservación Regional Ampiyacu Apayacu & -71.898781 & -3.094388 \\
\hline 72 & Datem del Marañón; Morona; Campamento Pongo Chinim (C1) & -77.775450 & -3.106180 \\
\hline 73 & Maynas; Napo; Tutapishcu, Rio Napo, left bank & -73.134528 & -3.115667 \\
\hline 74 & Maynas ; Mazan; Maijuna-Kichwa Regional Conservation Area & -72.931100 & -3.154420 \\
\hline 75 & Maynas; Mazan; Río Sucusari, Área de Conservación Regional Maijuna Kichwa & -72.936507 & -3.188581 \\
\hline 76 & Putumayo; Yaguas; Alto Cotuhé & -70.899028 & -3.198778 \\
\hline 77 & Mariscal Ramón Castilla; Pebas; Río Yaguasyacu, cuenca baja & -71.985188 & -3.209857 \\
\hline 78 & Maynas; Mazan; Caserío Juancho Playa, Río Napo & -72.833333 & -3.300000 \\
\hline 79 & Loreto; Tigre; Río Pucacuro, Cocha Coconilla, margen izquierda & -74.983333 & -3.316667 \\
\hline 80 & Loreto; Tigre; Río Pucacuro, Cocha Coconilla & -74.983300 & -3.316670 \\
\hline 81 & Mariscal Ramón Castilla; Pebas; Left bank Amazon, "Pevas"=Pebas & -71.817472 & -3.332972 \\
\hline 82 & Mariscal Ramón Castilla; Pebas; Mariscal Ramón Castilla & -71.816667 & -3.333333 \\
\hline 83 & Maynas; Alto Nanay; Río Nanay, Santa Luisa & -74.583333 & -3.333333 \\
\hline 84 & Mariscal Ramón Castilla; Pebas; Pevas, Amazonas, left bank & -71.816667 & -3.333333 \\
\hline 85 & Mariscal Ramón Castilla; Pebas; Peruvian Amazon & -71.827722 & -3.338889 \\
\hline 86 & Datem del Marañón; Morona; Río Morona, locality Nueva Alegría & -77.622606 & -3.362889 \\
\hline 87 & Loreto; Tigre; Tigre-Nanay & -75.239612 & -3.372517 \\
\hline 88 & Loreto; Tigre; Huanganayacu & -75.239612 & -3.372517 \\
\hline 89 & Loreto; Tigre; Río Huanganayacu-Río Tigre & -75.239612 & -3.372517 \\
\hline 90 & Maynas; Las Amazonas; Marupa Island, Amazon River & -72.666694 & -3.400000 \\
\hline 91 & Maynas; Indiana; Iquitos, 80 km R Maniti (=Río Maniti) & -72.850000 & -3.450000 \\
\hline 92 & Maynas; Las Amazonas; Río Apayacu, Amazonas, left bank & -72.300000 & -3.450000 \\
\hline 93 & Maynas; Las Amazonas; 1 km N Rio Napo, 157 river km NNE Iquitos & -72.750000 & -3.457620 \\
\hline 94 & Maynas; Iquitos; Río Nanay & -74.615850 & -3.463300 \\
\hline 95 & Maynas; Alto Nanay; Alto Nanay & -74.615850 & -3.463300 \\
\hline 96 & Maynas; Las Amazonas; Marupa River, Amazon (=Morupa) & -72.633333 & -3.466667 \\
\hline 97 & Maynas; Las Amazonas; Napo River & -72.750000 & -3.466667 \\
\hline 98 & Maynas; Las Amazonas; Quebrada Orán, ca. 5 km N Rio Amazonas, 85 km NE Iquitos & -72.517000 & -3.475000 \\
\hline
\end{tabular}




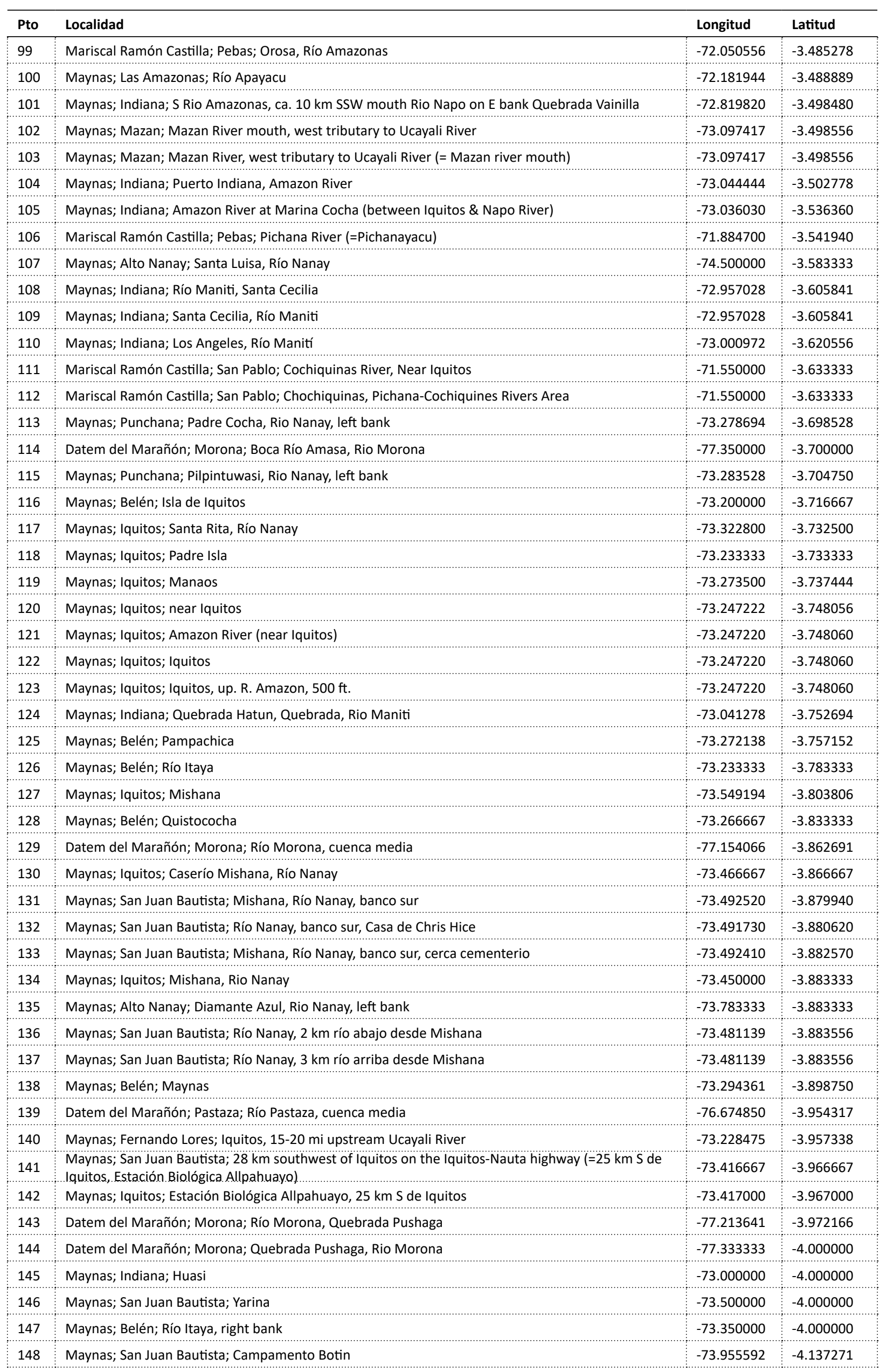


DIAZ ET AL.

\begin{tabular}{|c|c|c|c|}
\hline Pto & Localidad & Longitud & Latitud \\
\hline 149 & Mariscal Ramón Castilla; Yavarí; San Fernando & -70.233333 & -4.150000 \\
\hline 150 & Maynas; San Juan Bautista; Alto Itaya & -73.957758 & -4.155130 \\
\hline 151 & Maynas; San Juan Bautista; Belgica Km 60 carretera Iquitos-Nauta, O del camino & -73.482480 & -4.166570 \\
\hline 152 & Maynas; San Juan Bautista; El Triunfo km 47 carretera lquitos-Nauta, E del camino & -73.461700 & -4.169250 \\
\hline 153 & Mariscal Ramón Castilla; Yavarí; Río Yavari-Mirim, San Fernando & -70.284246 & -4.170245 \\
\hline 154 & Maynas; San Juan Bautista; La Habana km 52 carretera Iquitos-Nauta, Fundo Vargas, E del camino & -73.471930 & -4.185020 \\
\hline 155 & Maynas; San Juan Bautista; Quebrada Yanayacu & -73.708889 & -4.217778 \\
\hline 156 & Datem del Marañón; Pastaza; Río Chuinda, locality Puerto Requena & -76.778842 & -4.228248 \\
\hline 157 & Alto Amazonas; Pastaza; Huangana, aprox. $7.25 \mathrm{Kms}$ al NO de la boca del Río Pastaza & -76.568500 & -4.237470 \\
\hline 158 & Maynas; San Juan Bautista; Upper Río Itaya & -73.633333 & -4.250000 \\
\hline 159 & Maynas; San Juan Bautista; Cuenca del río Alto Itaya & -73.738509 & -4.251125 \\
\hline 160 & Maynas; San Juan Bautista; 2.5 km NE Villa Belén & -73.700000 & -4.262500 \\
\hline 161 & Maynas; San Juan Bautista; Carretera Iquitos-Nauta & -73.588194 & -4.277139 \\
\hline 162 & Loreto; Tigre; Río Tigre, just below Rio Tigrillo & -74.316667 & -4.283333 \\
\hline 163 & Loreto; Nauta; Río Tigre, 5 km above Río Tigrillo & -74.267860 & -4.302340 \\
\hline 164 & Loreto; Tigre; Mouth of Rio Tigrillo & -74.323889 & -4.312222 \\
\hline 165 & Loreto; Nauta; Río Tigre & -74.266667 & -4.316667 \\
\hline 166 & Loreto; Nauta; Río Tigre, 3 km above Rio Tigrillo & -74.266667 & -4.316667 \\
\hline 167 & Maynas; Fernando Lores; Hills 0.5 to $1 \mathrm{~km} \mathrm{E}$ and NE of San Pedro & -73.183333 & -4.316667 \\
\hline 168 & Maynas; Fernando Lores; Río Tahuayo & -73.229389 & -4.319833 \\
\hline 169 & Maynas; Fernando Lores; Quebrada Blanco, Río Tahuayo & -73.229389 & -4.319833 \\
\hline 170 & Loreto; Nauta; Río Tigre, 2 km above Rio Tigrillo & -74.274335 & -4.321629 \\
\hline 171 & Maynas; Fernando Lores; Quebrada Blanco, cuenca baja & -73.165293 & -4.324425 \\
\hline 172 & Loreto; Nauta; Río Tigre, 1 km above Rio Tigrillo & -74.279353 & -4.329265 \\
\hline 173 & Mariscal Ramón Castilla; Yavarí; Nueva Esperanza, Yavarí-Mirím & -71.959167 & -4.331389 \\
\hline 174 & Mariscal Ramón Castilla; Yavarí; Yavarí-Mirim River Basin & -71.959167 & -4.331389 \\
\hline 175 & Mariscal Ramón Castilla; Yavarí; Río Yavari Mirim, Quebrada Esperanza & -71.916667 & -4.333333 \\
\hline 176 & Loreto; Nauta; Río Tigrillo & -74.266667 & -4.333333 \\
\hline 177 & Mariscal Ramón Castilla; Yavarí; Quebrada Esperanza & -71.916667 & -4.333333 \\
\hline 178 & Loreto; Nauta; Nueva York, Rio Tigre, left bank & -74.199722 & -4.333833 \\
\hline 179 & Loreto; Nauta; Río Tigre, opposite Nueve York & -74.200000 & -4.337850 \\
\hline 180 & Maynas; Fernando Lores; Estación Biológica Quebrada Blanco & -73.150000 & -4.350000 \\
\hline 181 & Mariscal Ramón Castilla; Yavarí; Río Yavarí-Mirím, cuenca media & -72.260338 & -4.427242 \\
\hline 182 & $\begin{array}{l}\text { Maynas; Fernando Lores; Quebrada Blanco, cuenca alta, Área de Conservación Regional Tamshiyacu } \\
\text { Tahuayo }\end{array}$ & -73.065058 & -4.435068 \\
\hline 183 & $\begin{array}{l}\text { Mariscal Ramón Castilla; Yavarí; Río Yavarí, Lago Preto, Concesión de Conservación Lago Preto Pare- } \\
\text { dón }\end{array}$ & -71.708284 & -4.446088 \\
\hline 184 & Datem del Marañón; Manseriche; Puerto Melendez & -77.516667 & -4.450000 \\
\hline 185 & Requena; Saquena; Iquitos, Near; Ucayali River & -73.447188 & -4.451642 \\
\hline 186 & Mariscal Ramón Castilla; Yavarí; Lago Preto Conservation Concession & -71.765083 & -4.458472 \\
\hline 187 & Datem del Marañón; Manseriche; Pongo de Manseriche, Maranon River & -77.578694 & -4.461083 \\
\hline 188 & Loreto; Nauta; Lower Río Tigre & -74.086944 & -4.466667 \\
\hline 189 & Loreto; Nauta; Miraflores, Rio Marañon, left bank & -74.038611 & -4.480444 \\
\hline 190 & Mariscal Ramón Castilla; Yavarí; Río Yavarí-Mirím & -71.783056 & -4.482222 \\
\hline 191 & Loreto; Nauta; Nauta ca. Iquitos & -73.575630 & -4.505170 \\
\hline 192 & Loreto; Nauta; Nauta, Río Samiria; right bank & -73.550000 & -4.533333 \\
\hline 193 & Datem del Marañón; Manseriche; Saramiriza & -77.433333 & -4.550000 \\
\hline 194 & Loreto; Parinari; Lake Atun Cocha & -73.954250 & -4.560083 \\
\hline 195 & Loreto; Parinari; Parinari Canyon (=Parinari) & -74.433330 & -4.566660 \\
\hline 196 & Loreto; Parinari; Lake Tipishca de Samiria & -73.940139 & -4.579111 \\
\hline 197 & Loreto; Parinari; Parinari, Near, On Rio Maranon, Above Mouth of Rio Ucayali, Loreto & -74.500000 & -4.600000 \\
\hline 198 & Mariscal Ramón Castilla; Yavarí; Reserva Comunal Tamshiyacu Tahuayo & -72.993278 & -4.601139 \\
\hline
\end{tabular}




\begin{tabular}{|c|c|c|c|}
\hline Pto & Localidad & Longitud & Latitud \\
\hline 199 & Loreto; Nauta; Yanayaquillo, Samiria River & -73.904750 & -4.610111 \\
\hline 200 & Loreto; Parinari; Atun Caño & -73.938972 & -4.612694 \\
\hline 201 & Loreto; Nauta; San Jacinto & -73.987221 & -4.639017 \\
\hline 202 & Loreto; Parinari; Lake San Pablo de Tipishca & -73.800972 & -4.641222 \\
\hline 203 & Loreto; Parinari; Yanayacu River & -73.789528 & -4.649194 \\
\hline 204 & Loreto; Parinari; Pucate River & -73.837417 & -4.652444 \\
\hline 205 & Loreto; Parinari; Quebrada Yanayacu de Pucate & -73.837417 & -4.652444 \\
\hline 206 & Mariscal Ramón Castilla; Yavarí; Río Blanco & -73.000000 & -4.666667 \\
\hline 207 & Loreto; Parinari; Area de caza de Parinari & -74.557167 & -4.674056 \\
\hline 208 & Loreto; Parinari; Quebrada Parinari & -74.557167 & -4.674056 \\
\hline 209 & Loreto; Parinari; San Miguel, Río Samiria & -74.183417 & -4.693667 \\
\hline 210 & Loreto; Parinari; Area de caza de San Miguel & -74.183417 & -4.693667 \\
\hline 211 & Loreto; Parinari; Maranon River, Puerto Parinari & -74.350000 & -4.700000 \\
\hline 212 & Loreto; Parinari; Samiria River & -74.216667 & -4.700000 \\
\hline 213 & Loreto ; Parinari; Cocamilla, margen izquierda del Rio lanacu, frente a farinsua & -74.166667 & -4.816667 \\
\hline 214 & Datem del Marañón; Barranca; San Lorenzo & -76.600000 & -4.816670 \\
\hline 215 & Loreto; Parinari; Quebrada Pinche & -74.418167 & -4.840917 \\
\hline 216 & Loreto; Parinari; Quebrada Armana & -74.303139 & -4.887000 \\
\hline 217 & Loreto; Parinari; Río Samiria, Tacshacocha, Reserva Nacional Pacaya Samiria & -74.357455 & -4.891445 \\
\hline 218 & Loreto; Parinari; Cuenca del río Samiria & -74.569444 & -4.893472 \\
\hline 219 & Requena; Jenaro Herrera; Jenaro Herrera & -73.672790 & -4.904720 \\
\hline 220 & Loreto; Parinari; Tacsha Cocha & -74.066056 & -4.905944 \\
\hline 221 & Requena; Jenaro Herrera; Bretaña, Río Puinahua & -73.750000 & -4.916667 \\
\hline 222 & Loreto; Parinari; Quebrada Guanaico & -74.250361 & -4.953917 \\
\hline 223 & Loreto ; Parinari; Cocha Tacari, Rio lanacu & -74.216667 & -5.016667 \\
\hline 224 & Requena; Requena; Tapiche River & -73.850000 & -5.050000 \\
\hline 225 & Loreto; Parinari; Estación Biológica Pithecia & -74.666667 & -5.166667 \\
\hline 226 & Loreto; Parinari; Base Atun & -74.670000 & -5.170000 \\
\hline 227 & Loreto; Parinari; Río Samiria, Santa Elena & -74.750000 & -5.183330 \\
\hline 228 & Loreto; Parinari; Santa Elena, Río Samiria & -74.750000 & -5.183333 \\
\hline 229 & Loreto; Parinari; Katakari & -75.488889 & -5.188611 \\
\hline 230 & Requena; Yaquerana; Boca Río Yaquerana & -72.883333 & -5.200000 \\
\hline 231 & Loreto; Parinari; Hamburgo, Samiria River & -75.133333 & -5.200000 \\
\hline 232 & Loreto; Nauta; Reserva Nacional Pacaya Samiria & -74.591861 & -5.202556 \\
\hline 233 & Requena ; Yaquerana; Loboyacu & -73.409417 & -5.261250 \\
\hline 234 & Requena; Yaquerana; Nuevo San Juan, Río Gálvez & -73.163890 & -5.291670 \\
\hline 235 & Requena; Yaquerana; Quebrada Loboyacu, Reserva Nacional Matsés & -73.406809 & -5.334226 \\
\hline 236 & Requena; Puinahua; Estación Biológica Cahuana, Río Pacaya & -74.556409 & -5.353048 \\
\hline 237 & Requena; Puinahua; Isla Cahuana, Río Pacaya & -74.556409 & -5.353048 \\
\hline 238 & Requena; Requena; Quebrada Alemán, Reserva Nacional Matsés & -73.779978 & -5.420231 \\
\hline 239 & Requena ; Requena; Alemán & -73.780694 & -5.422889 \\
\hline 240 & Requena; Puinahua; Cahuana, Rio Pacaya & -74.566667 & -5.433333 \\
\hline 241 & Requena; Yaquerana; Choncó & -73.606111 & -5.556389 \\
\hline 242 & Requena; Soplin; Blanco River & -73.883900 & -5.578890 \\
\hline 243 & Requena; Yaquerana; Río Gálvez, Reserva Nacional Matsés & -73.526805 & -5.594960 \\
\hline 244 & Requena; Tapiche; San Pedro, Río Tapiche & -73.950000 & -5.633333 \\
\hline 245 & Requena ; Yaquerana; Alto Río Gálvez & -73.570639 & -5.646389 \\
\hline 246 & Alto Amazonas; Balsapuerto; Alto Cahuapanas (1000-1400m) & -76.833333 & -5.650000 \\
\hline 247 & Requena; Tapiche; Río Tapiche & -74.003611 & -5.655000 \\
\hline 248 & Alto Amazonas; Balsapuerto; Mina de Sal (300-700m) & -76.600000 & -5.666667 \\
\hline
\end{tabular}


DIAZ ET AL.

\begin{tabular}{|c|c|c|c|}
\hline Pto & Localidad & Longitud & Latitud \\
\hline 249 & Alto Amazonas; Balsapuerto; Baradero, Samiria River (=Baradero) & -76.450000 & -5.733333 \\
\hline 250 & Requena; Emilio San Martín; Ucayali River & -74.456917 & -5.744111 \\
\hline 251 & Loreto; Urarinas; Marañón River & -74.456917 & -5.744111 \\
\hline 252 & Requena; Tapiche; San Pedro, Quebrada Yanayacu, Río Tapiche & -74.036941 & -5.830963 \\
\hline 253 & Alto Amazonas; Balsapuerto; Balsapuerto & -76.600000 & -5.833333 \\
\hline 254 & Alto Amazonas; Yurimaguas; Puerto Arturo & -76.050000 & -5.833333 \\
\hline 255 & Requena; Soplin; Itia Tebu & -73.760278 & -5.858333 \\
\hline 256 & Alto Amazonas; Balsapuerto; Alto Cachiyacu (500-1950m) & -76.700000 & -5.883333 \\
\hline 257 & Alto Amazonas; Yurimaguas; ca. Yurimaguas & -76.106910 & -5.893940 \\
\hline 258 & Alto Amazonas; Yurimaguas; Yurimaguas & -76.106910 & -5.893940 \\
\hline 259 & Requena; Soplin; Río Blanco, right bank & -73.705722 & -5.900833 \\
\hline 260 & Requena; Alto Tapiche; Puerto Punga, Tapiche River & -74.033333 & -6.233333 \\
\hline 261 & Requena; Yaquerana; Actiame & -73.157778 & -6.317500 \\
\hline 262 & Requena; Alto Tapiche; Río Ucayali & -74.032670 & -6.696670 \\
\hline 263 & Ucayali; Vargas Guerra; Sarayacu, Ucayali River & -75.106111 & -6.817778 \\
\hline 264 & Ucayali; Vargas Guerra; Campamento El Fuerte (Cerros de Orellana) & -75.156910 & -6.908810 \\
\hline 265 & Ucayali; Inahuaya; Upuano (Inahuaya) & -75.244970 & -7.094890 \\
\hline 266 & Ucayali; Inahuaya; Inahuaya & -75.264130 & -7.115770 \\
\hline 267 & Ucayali; Inahuaya; Campamento Venado, bajo Inaguaiyo (=Inahuaya) & -75.283417 & -7.149972 \\
\hline 268 & Ucayali; Contamana; Aguas Calientes (Cerros de Contamana) & -74.948250 & -7.188920 \\
\hline 269 & Ucayali; Contamana; Sierra de Contamana, Cerros de Canchaguaya, Aguas Calientes & -74.948250 & -7.188920 \\
\hline 270 & Ucayali; Pampa Hermosa; Cushabatay, Ucayali River & -75.269722 & -7.211944 \\
\hline 271 & Ucayali; Contamana; Aguas Calientes, Contamana, Rio Ucayali, right bank & -74.950000 & -7.216667 \\
\hline 272 & Requena; Alto Tapiche; Ucayali, Cerros de Contamana & -74.085472 & -7.224861 \\
\hline 273 & Requena; Alto Tapiche; Cordillera Azul & -74.566667 & -7.233333 \\
\hline 274 & Requena; Alto Tapiche; Contamana, Cerro Azul & -74.566667 & -7.233333 \\
\hline 275 & Requena; Alto Tapiche; Cerro Azul, Contamana, east bank Rio Ucayali & -74.566667 & -7.233333 \\
\hline 276 & Ucayali; Contamana; Contamana, Ucayali River & -74.900000 & -7.250000 \\
\hline 277 & Ucayali ; Contamana; El Indio (Cerros de Contamana) & -74.900000 & -7.250000 \\
\hline 278 & Ucayali; Contamana; Campamento III, Sierra de Contamana & -74.682924 & -7.282584 \\
\hline 279 & Requena; Alto Tapiche; Mouth of Rio Bombo also upper Tapiche, $800 \mathrm{ft}$. & -73.916667 & -7.316417 \\
\hline 280 & Ucayali; Contamana; Campamento II, Sierra de Contamana & -74.728193 & -7.327842 \\
\hline 281 & Ucayali; Contamana; Sierra de Contamana & -74.728193 & -7.327842 \\
\hline 282 & Ucayali; Contamana; Bahuacuraya, west bank of mid Ucayali River, opposite Contamana & -75.014972 & -7.363833 \\
\hline 283 & Ucayali; Contamana; Campamento I, Sierra de Contamana & -74.818777 & -7.373117 \\
\hline 284 & Ucayali ; Contamana; Yapatio River & -75.367444 & -7.411111 \\
\hline 285 & Ucayali; Contamana; Río Cashiboya, Parque Nacional Sierra Divisor & -74.737009 & -7.461837 \\
\hline 286 & Ucayali; Contamana; Quebrada de Inuya & -74.931100 & -7.515000 \\
\hline 287 & Ucayali; Pampa Hermosa; Pauya & -75.933333 & -7.586083 \\
\hline 288 & Ucayali ; Padre Marquez; Roaboya, Ucayali River & -74.866667 & -7.800000 \\
\hline 289 & Ucayali ; Contamana; Pisqui River, west tributary of Ucayali River & -75.701444 & -8.404306 \\
\hline 290 & Requena; Alto Tapiche; Divisor & -73.882861 & -7.204556 \\
\hline 291 & Requena; Alto Tapiche; Tapiche & -73.934472 & -7.208472 \\
\hline 292 & Requena; Alto Tapiche; Ojo de Contaya & -74.588500 & -7.1159722 \\
\hline
\end{tabular}


Anexo 2 Mapas de distribución de las 72 especies de mamíferos mayores de Loreto.
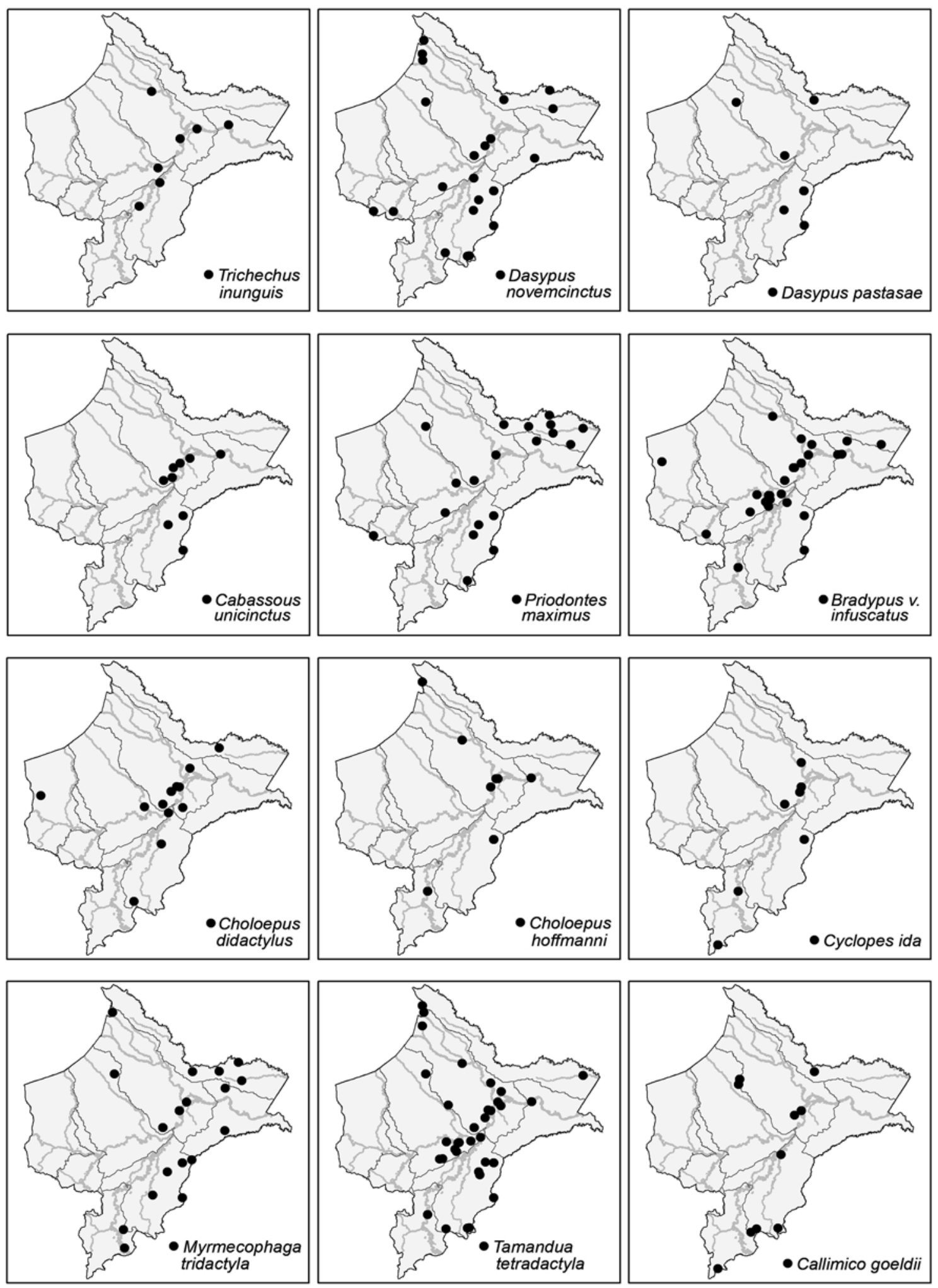

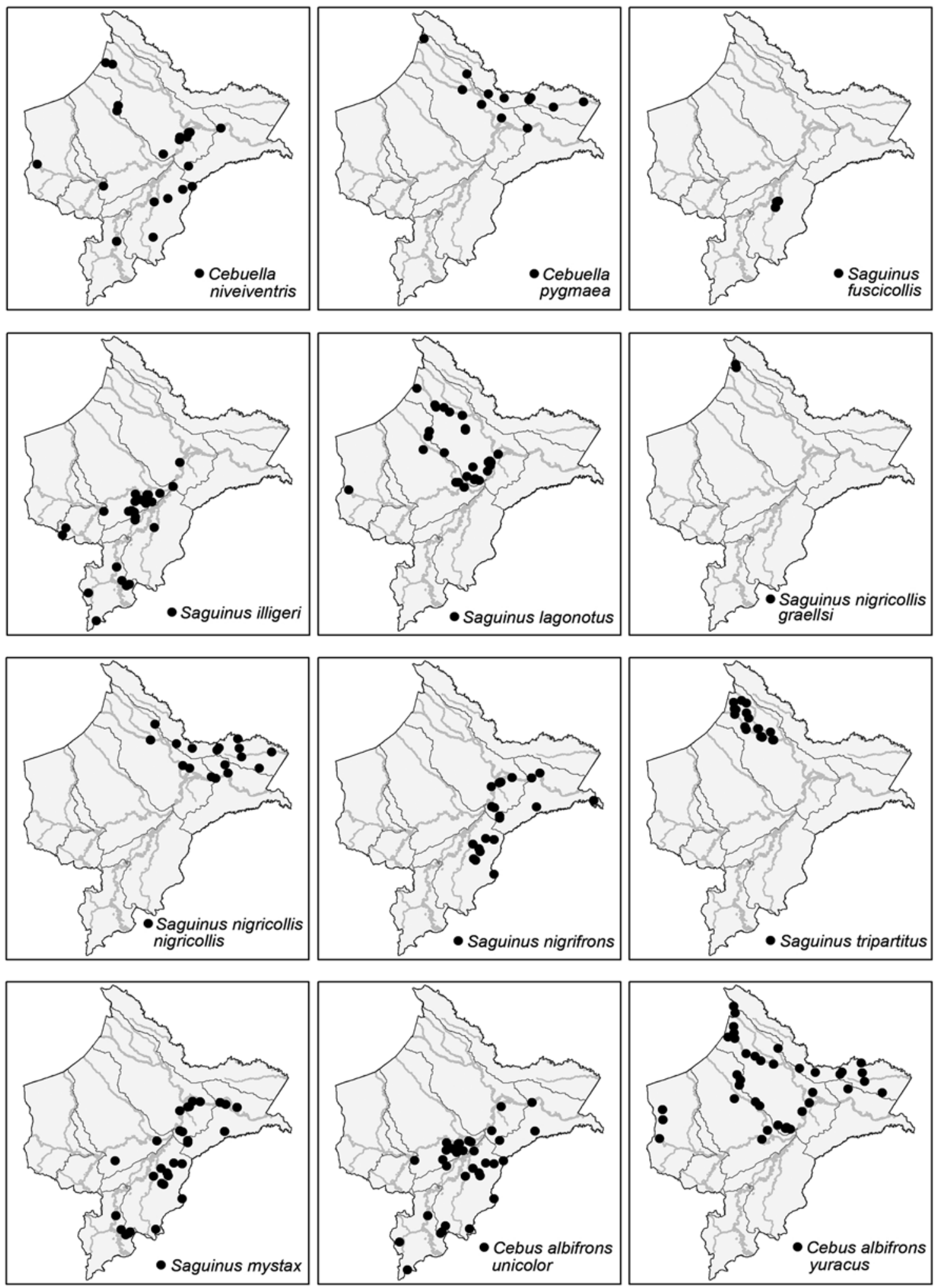

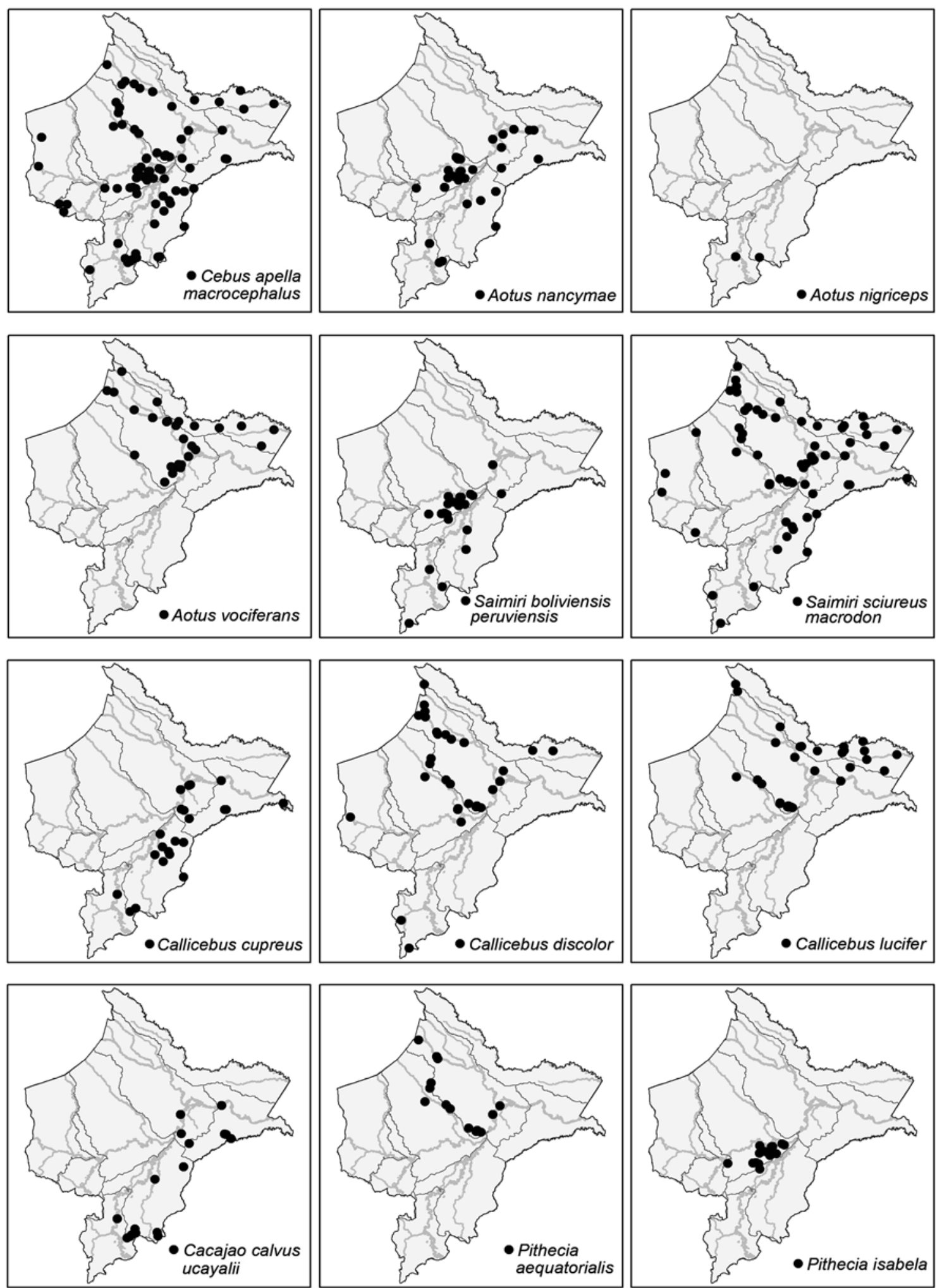

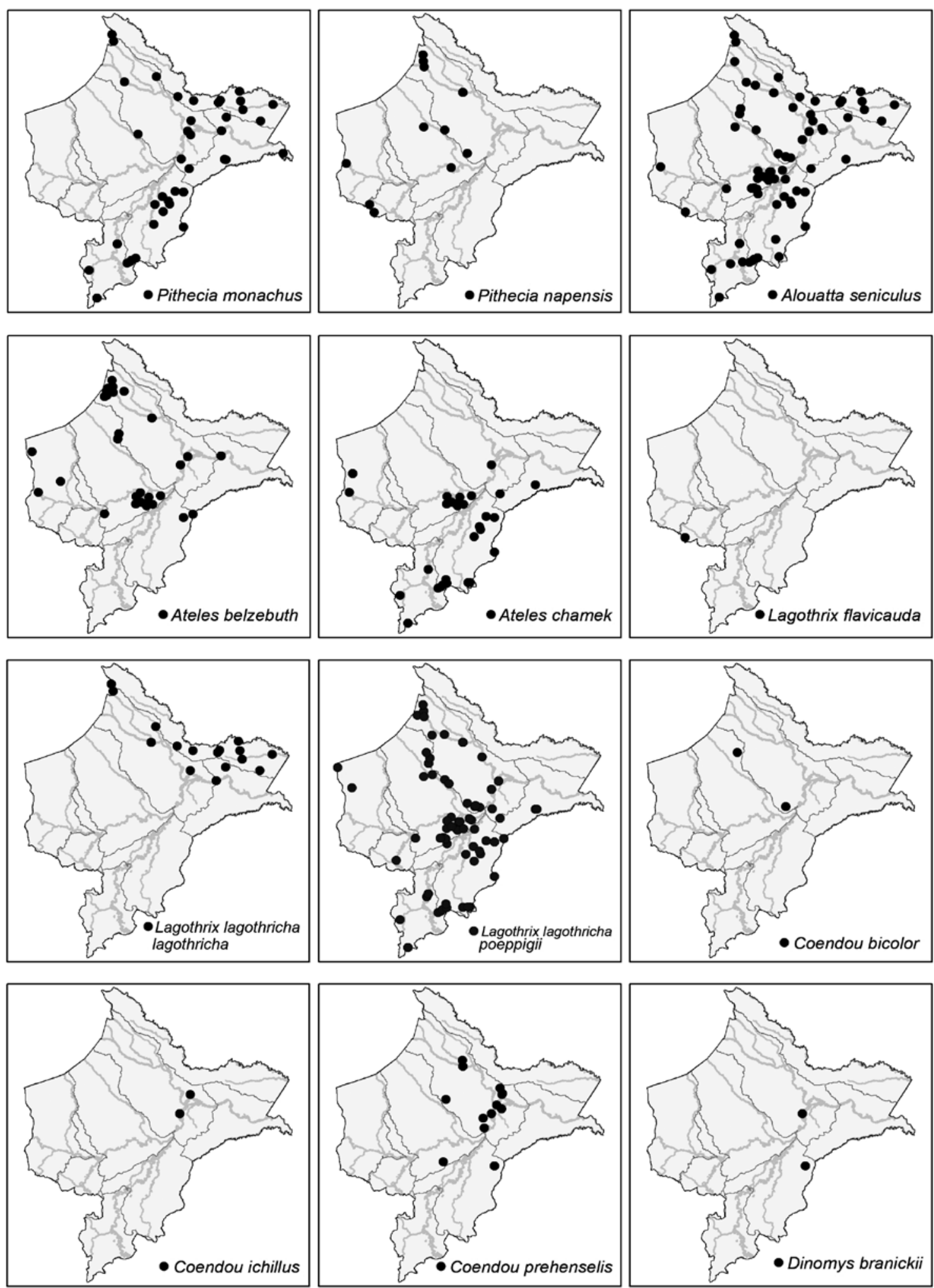

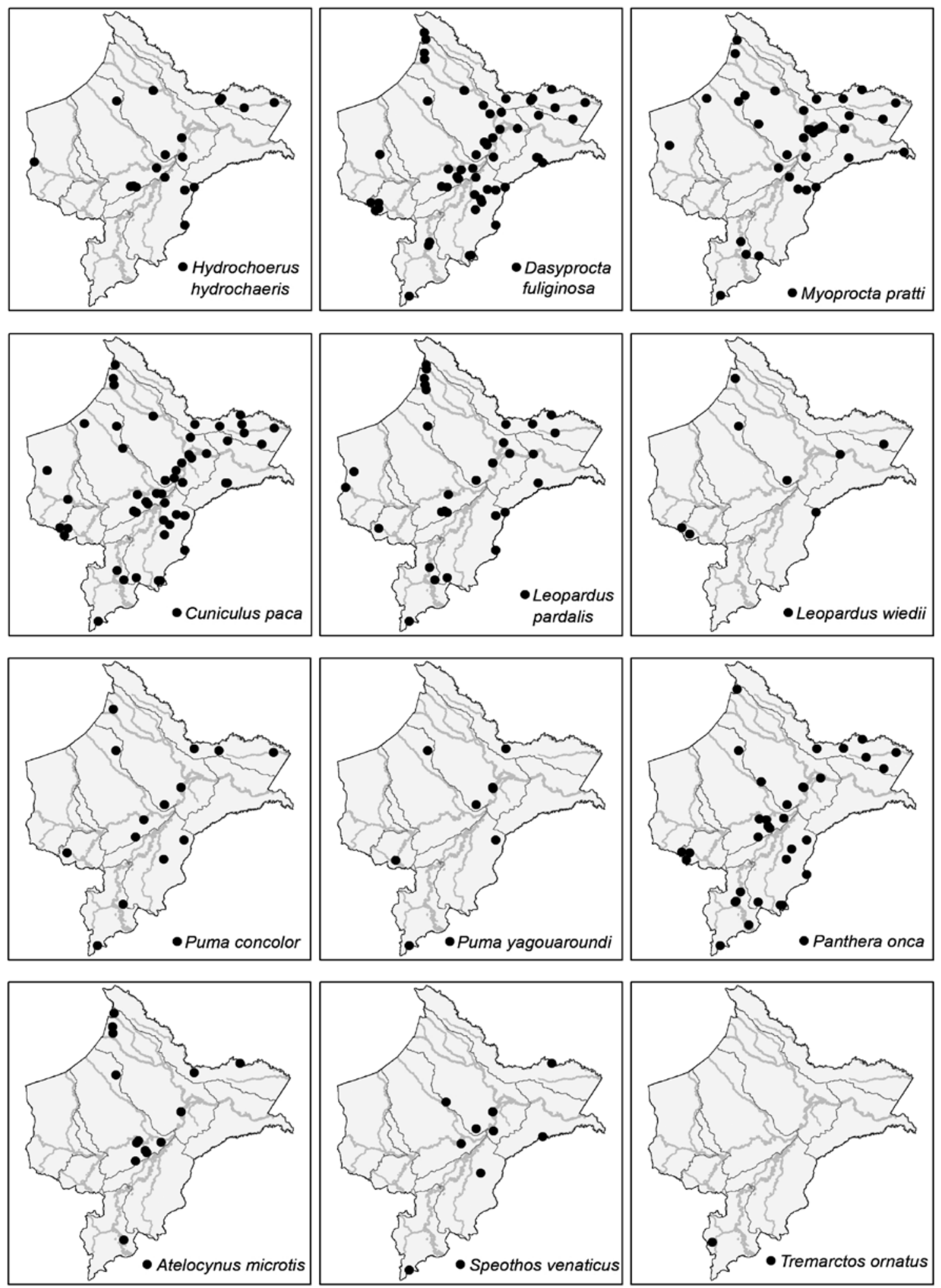

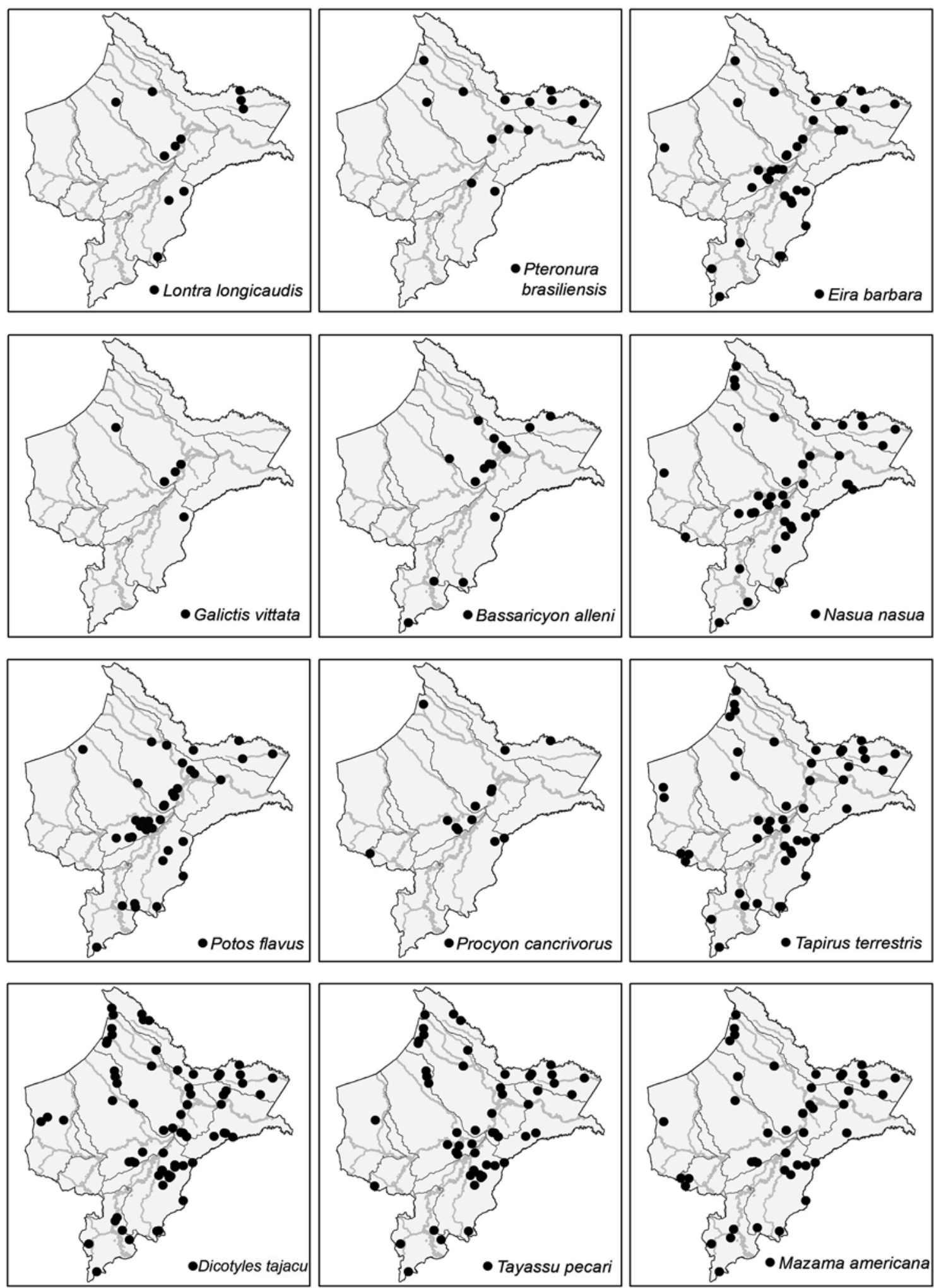

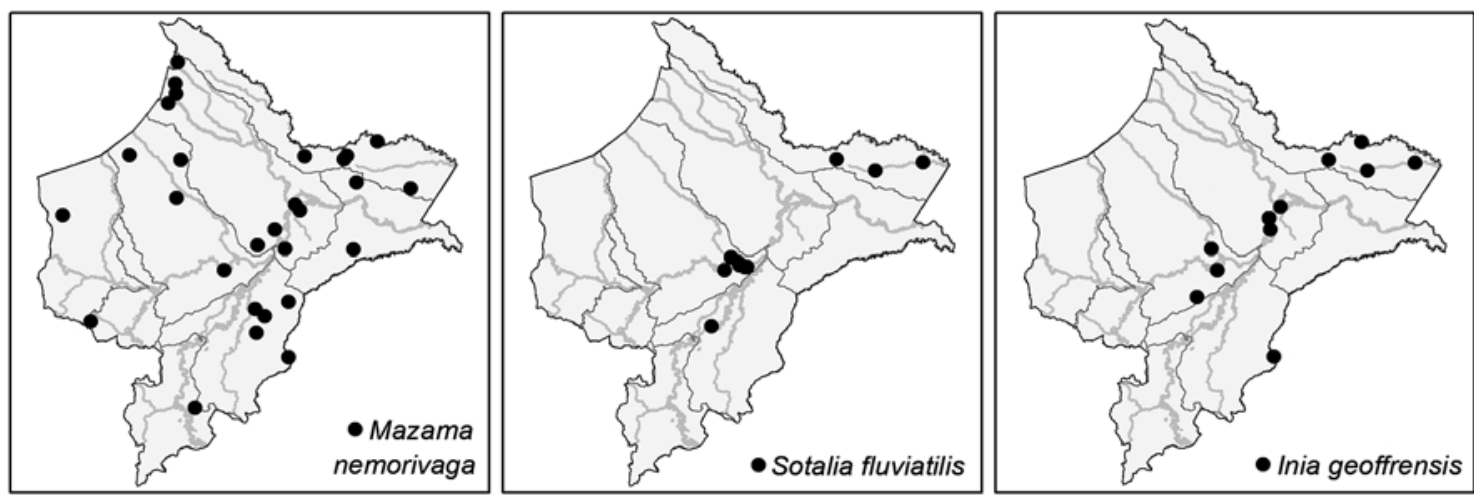

Anexo 3 Referencias que sustentan la presencia de cada especie en las localidades del Anexo 1.

\section{Trichechus inunguis}

Loreto.- 199: AMNH 188221; 251: Santizábal et al. 2012. Mariscal Ramón Castilla.- 85: Santizábal et al. 2012. Maynas.- 38: AMNH 73594, 97: Santizábal et al. 2012, 122: AMNH 169940. Requena.- 224: AMNH 98691; 250: Santizábal et al. 2012.

Dasypus novemcinctus

Alto Amazonas.- 256: Patterson y López-Wong 2014; 258: MUSM 2052. Loreto.- 55: Falcón-Ayapi et al. 2019; 225: FMNH 123022. Mariscal Ramón Castilla.- 173: Mayor et al. 2015. Maynas.- 3: Pérez-Peña et al. 2019; 10: Pérez-Peña et al. 2019; 12: Pérez-Peña et al. 2019; 122: AMNH 98458 , AMNH 98462, AMNH 98463, AMNH 98465, AMNH 98515, AMNH 991734; 142: MUSM 16502; 159: Navarro y Terrones 2006, Aquino et al. 2012b. Putumayo.- 37: Bravo et al. 2016; 52: Bravo et al. 2016; 62: Montenegro y Moya 2011. Requena.- 221: Saldaña y Saldaña 2011; 234: Fleck y Harder 2000, Voss y Fleck 2017; 241: Amanzo 2006; 255: Amanzo 2006; 261: Amanzo 2006; 290: Jorge y Velazco 2006; 291: Jorge y Velazco 2006; 292 : Jorge y Velazco 2006.

\section{Dasypus pastasae}

Loreto.- 55: Falcón-Ayapi et al. 2019. Maynas.- 159: Aquino et al. 2012b. Putumayo.- 52: Bravo et al. 2016. Requena.- 234: Fleck y Harder 2000, Voss y Fleck 2017, 255: Amanzo 2006, 261: Amanzo 2006.

\section{Cabassous unicinctus}

Mariscal Ramón Castilla.- 99: Voss y Fleck 2017. Maynas.- 108: Voss y Fleck 2017; 122: Anacleto et al. 2013; 134: FMNH 112562; 151: MUSM 33409; 159: Aquino et al. 2012b. Requena.- 234: Fleck y Harder 2000; 241: Amanzo 2006; 261: Amanzo 2006.

\section{Priodontes maximus}

Alto Amazonas.- 256: Patterson y López-Wong 2014. Loreto.- 55: FalcónAyapi et al. 2019, 176: KU 130343; 232: Pittet y Bennett 2014. Mariscal Ramón Castilla.- 54: Bravo et al. 2016, 71: Puertas et al. 2017. Maynas.102: AMNH 98460-1; 159: Aquino et al. 2012b, Navarro y Terrones 2006. Putumayo.- 37: Bravo et al. 2016; 52: Bravo et al. 2016; 53: Montenegro y Moya 2011, 56: Montenegro y Moya 2011; 62: Montenegro y Moya 2011; 76: Montenegro y Moya 2011. Requena.- 234: Fleck y Harder 2000, Voss y Fleck 2017; 241: Amanzo 2006; 255: Amanzo 2006; 261: Amanzo 2006; 291: Jorge y Velazco 2006.

\section{Bradypus variegatus infuscatus}

Alto Amazonas.- 254: Voss y Fleck 2017; Datem del Marañón.- 114: FMNH 88893. Loreto.- 205: Aquino 2005; 207: Aquino y Calle 2003; 208: Aquino 2005; 210: Aquino y Calle 2003; 212: AMNH 188193-6; 213: Voss y Fleck 2017; 216: Aquino 2005; 222: Aquino 2005; 223: Voss y Fleck 2017; 228: Voss y Fleck 2017. Mariscal Ramón Castilla.- 71: Puertas et al. 2017, 99: Voss y Fleck 2017. Maynas.- 38: AMNH 71822; 68: LSUMZ 28396; 75: Bowler et al. 2016; 100: Voss y Fleck 2017; 104: Voss y Fleck 2017; 122: AMNH 98532-3, 98536, 98542, 98545-6, 98820; 134: Voss y Fleck 2017, 136: MUSM 33611; 159: Aquino et al. 2007a, Aquino et al. 2012b, Navarro y Terrones 2006. Putumayo.- 76: Montenegro y Moya 2011. Requena.- 219: Voss y Fleck 2017; 234: Voss y Fleck 2017; 261: Amanzo 2006. Ucayali.263: Voss y Fleck 2017.

\section{Choloepus didactylus}

Datem del Marañón.- 144: FMNH 88892. Loreto.-172: FMNH 123021; 191: MUSM 1316. Maynas.- 75: Bowler et al. 2016; 117: FMNH 86893; 122: McAfee 2014; 133: MUSM 33612-4; 137: MUSM 33615; 180: Heymann et al. 2011; 159: Aquino et al. 2012b. Putumayo.- 50: Aquino et al. 2007b. Requena.- 239: Torres-Oyarce et al. 2017; 292: Jorge y Velazco 2006.

Choloepus hoffmanni

Mariscal Ramón Castilla.- 99: Voss y Fleck 2017. Maynas.- 1: AMNH 71820-1; 38: AMNH 71823-4; 102: AMNH 98543; 104: AMNH 73567-70, 73755-6; 122: AMNH 146841, 98531, 98534-5, 98537, 98540-1, 98547. Requena.- 234: Fleck y Harder 2000. Ucayali.- 263: AMNH 76404-7.

Cyclopes ida

Maynas.- 68: LSUMZ 34955; 122: AMNH 204662, 98518, 98521, 98523-5, 99199, FMNH 89172; 138: Coimbra et al. 2017; 159: Navarro y Terrones 2006. Requena.- 234: AMNH 268232, MUSM 11092-3. Ucayali.- 263: AMNH 75281; 289: AMNH 98519-20, 98526-7, 9885.

Myrmecophaga tridactyla

Loreto.- 55: Falcón-Ayapi et al. 2019. Mariscal Ramón Castilla.- 71: Puertas et al. 2017; 177: Voss y Fleck 2017. Maynas.- 3: Pérez-Peña et al. 2019; 104: AMNH 73588; 122: AMNH 98507-8, 98510, 98514; 159: Aquino et al. 2007a, Aquino et al. 2012b, Navarro y Terrones 2006. Putumayo.- 37: Bravo et al. 2016; 50: Aquino et al. 2007b; 52: Bravo et al. 2016; 62: Montenegro y Moya 2011. Requena.- 230: Voss y Fleck 2017; 234: Fleck y Harder 2000, Voss y Fleck 2017; 241: Amanzo 2006; 260: AMNH 98511; 261: Amanzo 2006. Ucayali.- 276: AMNH 98506; 288: AMNH 98513.

\section{Tamandua tetradactyla}

Loreto.- 55: Falcón-Ayapi et al. 2019; 192: KU 139388; 205: Aquino 2005; 207: Aquino y Calle 2003; 208: Aquino 2005; 210: Aquino y Calle 2003; 212: AMNH 188191; 216: Aquino 2005; 222: Aquino 2005; 225: FMNH 123020; 228: FMNH 86891. Mariscal Ramón Castilla.- 99: Voss y Fleck 2017. Maynas.- 1: AMNH 71818-9; 3: Pérez-Peña et al. 2019; 10: Pérez-Peña et al. 2019; 38: AMNH 73577; 64: LSUMZ 28419; 75: Bowler et al. 2016; 104: AMNH 73753-4 107: FMNH 86890; 109: Voss y Fleck 2017; 117: FMNH 86889; 122: AMNH 98504-5, 98573, 99188; 142: MUSM 16504; 159: Aquino et al. 2007a, Aquino et al. 2012b, Navarro y Terrones 2006. Putumayo.- 56: Montenegro y Moya 2011. Requena.- 233: Torres-Oyarce et al. 2017; 234: Voss y Fleck 2017; 241: Amanzo 2006; 245: Torres-Oyarce et al. 2017; 261: Amanzo 2006; 274: FMNH 64289; 290: Jorge y Velazco 2006; 291: Jorge y Velazco 2006. Ucayali.- 263: AMNH 75293-4.

\section{Callimico goeldii}

Loreto.- 61: Pérez-Peña et al. 2016; 65: Pérez-Peña et al. 2016. Maynas.- 122: AMNH 183284-5, 99249; 134: FMNH 60354. Putumayo.- 52: Bravo et al. 2016. Requena.- 224: AMNH 98281; 273: Hershkovitz 1977; 291: Jorge y Velazco 2006. Ucayali.- 281: Aquino et al. 2005a; 289: AMNH 98367.

\section{Cebuella niveiventris}

Datem del Marañón.- 193: Vermeer et al. 2013. Loreto.- 61: Pérez-Peña et al. 2016; 65: Pérez-Peña et al. 2016; 229: Allgas et al. 2018. Mariscal Ramón Castilla.- 99: Voss y Fleck 2011; 198: Puertas et al. 1995. Maynas.-15: Aquino et al. 2014a; 19: Aquino et al. 2014a; 109: Garbino et al. 2019, Glander et 
al. 1984; 110: Glander et al. 1984; 122: AMNH 98487, 99306; 124: Soini 1982; 128: Garbino et al. 2019; 159: Aquino et al. 2012b, Navarro y Terrones 2006. Requena.- 230: Voss y Fleck 2011, 234: Garbino et al. 2019, Voss y Fleck 2011; 241: Amanzo 2006; 247: Bennet et al. 2001; 262: UMMZ 62505. Ucayali.- 263: Garbino et al. 2019.

\section{Cebuella pygmaea}

Mariscal Ramón Castilla.- 54: Aquino et al. 2016. Maynas.- 3. Pérez-Peña et al. 2019; 24: Aquino et al. 2016; 38: AMNH 72033-9; 59: AMNH 98516-7; 75: Bowler et al. 2016; 100: AMNH 74360-3, 74401. Putumayo.- 42: Aquino et al. 2016; 50: Aquino et al. 2007b; 52: Bravo et al. 2016; 56: Montenegro y Moya 2011; 62: Montenegro y Moya 2011.

Saguinus (Leontocebus) fuscicollis

Requena.- 244: FMNH 123037-9, 123044-5; 247: Bennet et al. 2001, Matauschek 2010; 252: FMNH 123040-3.

\section{Saguinus (Leontocebus) illigeri}

Alto Amazonas.- 248: Patterson y López-Wong 2014; 256: Patterson y López-Wong 2014. Loreto.- 205: Aquino 2005; 208: Aquino 2005; 209: Aquino 2005; 210: Aquino y Calle 2003; 212: AMNH 188165-70; 215: Aquino 2005; 216: Aquino 2005; 218: Aquino y Bodmer 2004; 220 : Aquino 2005; 222: Aquino 2005; 225: Pacheco y Pezo 1982; 228: FMNH 87145-7; 229: Allgas et al. 2018; 232: Pittet y Bennett 2014. Maynas.- 123: AMNH 98278-80. Requena.- 185: USNM 336299-301, 337331; 237: Soini 1986; 240: Matauschek 2010; 247: Matauschek 2010. Ucayali.- 263: AMNH 976, 76337, 76340-1, 76345, 76351, 76354, 76356, 76358, 76362-3, 76366-70; 271: Matauschek 2010; 280: Aquino et al. 2005a; 283: Aquino et al. 2005a; 287: Pacheco y Arias 2001; 289: Pacheco y Arias 2001.

\section{Saguinus (Leontocebus) lagonotus}

Datem del Marañón.- 193: Vermeer et al. 2013. Loreto.- 61: Pérez-Peña et al. 2016; 65: Pérez-Peña et al. 2016; 88: Aquino et al. 2015; 172: FMNH 122756-8; 178: Matauschek 2010; 189: Matauschek 2010. Maynas.- 15: Aquino et al. 2014a; 28: Heymann et al. 2002; 29: Heymann et al. 2002; 31: Heymann et al. 2002; 35: Heymann et al. 2002; 38: AMNH 73389-402; 57 : Matauschek 2010; 60: Matauschek 2010; 95: Aquino et al. 2015; 104: AMNH 73725-740; 113: Matauschek 2010; 115: Matauschek 2010; 117: FMNH 86963, 87143-4; 122: AMNH 98821, MUSM 37-38; 125: FMNH 87141-2; 128: FMNH 122753; 135: Matauschek 2010; 147: Matauschek 2010; 150: Aquino et al. 2015; 155: Charpentier et al. 2015; 159: Aquino et al. 2007a, Aquino et al. 2012b, Navarro y Terrones 2006; 161: Aquino et al. 2014b.

Saguinus (Leontocebus) nigricollis graellsi

Maynas.- 3: Pérez-Peña et al. 2019; 4: Matauschek 2010.

\section{Saguinus (Leontocebus) nigricollis nigricollis}

Mariscal Ramón Castilla.- 54: Aquino et al. 2016, Bravo et al. 2016; 71: Puertas et al. 2017; 81: AMNH 98290-1; 84: Matauschek 2010. Maynas.- 24: Aquino et al. 2016; 38: AMNH 72040-55; 73: Matauschek 2010; 75: Bowler et al. 2016; 92: Matauschek 2010; 100: AMNH 74402-15. Putumayo.- 37: Bravo et al. 2016; 42: Aquino et al. 2016; 50: Aquino et al. 2007b, 52: Bravo et al. 2016; 53: Montenegro y Moya 2011; 56: Montenegro y Moya 2011; 62: Montenegro y Moya 2011; 76: Montenegro y Moya 2011.

\section{Saguinus (Leontocebus) nigrifrons}

Mariscal Ramón Castilla.- 82: FMNH 35056; 99: AMNH 73742, 73746, 73748-9, 73984, 74038, 74040-6, 74048, 74051, 74053; 149: FMNH 888734; 177: FMNH 88874; 198: Puertas et al. 1995; 206: Heymann 1990a. Maynas.- 96: AMNH 98292, 98294-6 109: Glander et al. 1984; 110: Glander et al. 1984; 118: Nickle y Heymann 1996; 168: Heymann y Aquino 1994; 180 : Heymann 1995, Matauschek 2010, Nadjafzadeh y Heymann 2008, Nickle y Heymann 1996. Requena.- 233: Torres-Oyarce et al. 2017; 234: Fleck y Harder 2000, Voss y Fleck 2011, 2017; 239: Torres-Oyarce et al. 2017; 245: Torres-Oyarce et al. 2017; 241: Amanzo 2006; 255: Amanzo 2006; 259: Matauschek 2010; 261: Amanzo 2006.

\section{Saguinus (Leontocebus) tripartitus}

Maynas.- 9: Matauschek 2010, Rylands et al. 2011; 10: Pérez-Peña et al. 2019; 11: Aquino y Encarnación 1996; 12: Pérez-Peña et al. 2019; 13: Aquino y Encarnación 1996; 16: Aquino et al. 2005b; 19: Aquino et al. 2014a; 22: Aquino et al. 2005b; 25: Matauschek 2010; 26: Aquino y Encarnación 1996; 27: Aquino y Encarnación 1996; 28: Aquino y Encarnación 1996, Heymann et al. 2002; 30: Aquino y Encarnación 1996; 34: Aquino y Encarnación 1996; 35: Heymann et al. 2002; 36: Aquino y Encarnación 1996; 38: AMNH 72091-100, AMNH 72133-7; 39: Aquino y Encarnación 1996.

\section{Saguinus (Tamarinus) mystax}

Loreto.- 199: AMNH 188174, 188176; 231: AMNH 188171. Mariscal Ramón Castilla.- 99: Voss y Fleck 2011; 106: USNM 543488-90; 111: USNM 543132, 543485-7, 544381-3; 112: USNM 543133-6; 177: Voss y Fleck
2011; 198: Puertas et al. 1995; 206: Heymann 1990a. Maynas.- 91: MUSM 1844-5; 96: Voss y Fleck 2011; 109: Glander et al. 1984, Voss y Fleck 2011; 110: Glander et al. 1984; 118: Heymann1990b, Nickle y Heymann 1996; 168: Heymann y Aquino 1994; 180: Heymann 1995, Matauschek 2010, Nadjafzadeh y Heymann 2008, Nickle y Heymann 1996. Requena.- 233: Torres-Oyarce et al. 2017; 234: Fleck y Harder 2000, Voss y Fleck 2011; 239: Torres-Oyarce et al. 2017; 241: Amanzo 2006; 245: Torres-Oyarce et al. 2017; 247: Matauschek 2010; 255: Amanzo 2006; 259: Matauschek 2010; 261: Amanzo 2006; 291: Jorge y Velazco 2006. Ucayali.- 263: AMNH 76330, 76332, 76335-6, 76339, 76343-4, 76353, 76357, 76359, 76361, 271: Matauschek 2010; 278: Aquino et al. 2005a; 280: Aquino et al. 2005a; 283: Aquino et al. 2005a.

\section{Cebus (Cebus) albifrons unicolor}

Loreto.- 199: AMNH 188014-7, 188019-20; 205: Aquino 2005; 207: Aquino y Calle 2003; 208: Aquino 2005; 209: Aquino 2005; 210: Aquino y Calle 2003; 212: AMNH 188013, 188018, 188021; 215: Aquino 2005; 216 : Aquino 2005; 218: Aquino y Bodmer 2004; 220: Aquino 2005; 222: Aquino 2005; 225: Pacheco y Pezo 1982; 229: Allgas et al. 2018. Mariscal Ramón Castilla.- 99: Voss y Fleck 2011; 173: Mayor et al. 2015; 174: Conga et al. 2018; 198: Puertas et al. 1995. Maynas.- 109: Voss y Fleck 2011; 168: Heymann y Aquino 1994. Requena.- 221: Saldaña y Saldaña 2011; 230: Voss y Fleck 2011; 233: Torres-Oyarce et al. 2017; 234: Voss y Fleck 2011; 237: Soini 1986; 239: Torres-Oyarce et al. 2017; 241: Amanzo 2006; 245: TorresOyarce et al. 2017; 247: Bennet et al. 2001; 261: Amanzo 2006; 290: Jorge y Velazco 2006; 292: Jorge y Velazco 2006. Ucayali.- 263: AMNH 76398, 76416, 76492, 76493; 278: Aquino et al. 2005a; 280: Aquino et al. 2005a; 287: Pacheco y Arias 2001; 289: Pacheco y Arias 2001.

\section{Cebus (Cebus) albifrons yuracus}

Datem del Marañón.- 114: FMNH 88852-3; 144: FMNH 88849-51; 193 . Vermeer et al. 2013. Loreto.- 55: Falcón-Ayapi et al. 2019; 61: Pérez-Peña et al. 2016; 65: Pérez-Peña et al. 2016; 88: Aquino et al. 2015; 163: FMNH 122795; 195: AMNH 188022-3. Mariscal Ramón Castilla.-54: Aquino et al. 2016, Bravo et al. 2016; 71: Puertas et al. 2017. Maynas.- 1: AMNH 71813, 71815; 3: Pérez-Peña et al. 2019; 10: Pérez-Peña et al. 2019; 12: Pérez-Peña et al. 2019; 15: Aquino et al. 2014a; 19: Aquino et al. 2014a; 24: Aquino et al. 2016; 28: Heymann et al. 2002; 31: Heymann et al. 2002; 35: Heymann et al. 2002; 38: AMNH 71781, 71795-801; 75: Bowler et al. 2016; 95: Aquino et al. 2015; 104: AMNH 73563-6; 107: FMNH 86929-31; 122: AMNH 98349, 98359; 150: Aquino et al. 2015; 155: Charpentier et al. 2015; 159: Aquino et al. 2007a, Aquino et al. 2009a, 2012b, Navarro y Terrones 2006; 161: Aquino et al. 2014b. Putumayo.-37: Bravo et al. 2016; 42: Aquino et al. 2016; 50: Aquino et al. 2007b; 52: Bravo et al. 2016; 53: Montenegro y Moya 2011; 62: Montenegro y Moya 2011; 76: Montenegro y Moya 2011.

\section{Cebus (Sapajus) apella macrocephalus}

Alto Amazonas.- 246: Patterson y López-Wong 2014; 248: Patterson y LópezWong 2014; 256: Patterson y López-Wong 2014. Datem del Marañón.- 114: FMNH 88844-5; 193: Vermeer et al. 2013. Loreto.- 55: Falcón-Ayapi et al. 2019; 61: Pérez-Peña et al. 2016; 65: Pérez-Peña et al. 2016; 80: MUSM 17556; 88: Aquino et al. 2015; 163: FMNH 122794; 172: FMNH 122793; 195: AMNH 188051; 199 AMNH 188030-6, 188041-8; 205: Aquino 2005; 207: Aquino y Calle 2003; 208: Aquino 2005; 209: Aquino 2005; 210 : Aquino y Calle 2003; 212 : AMNH 188025-8; 215: Aquino 2005; 216 : Aquino 2005; 218: Aquino y Bodmer 2004; 220: Aquino 2005, 222: Aquino 2005; 225: Pacheco y Pezo 1982; 228: FMNH 86938-9; 229: Allgas et al. 2018; 231: AMNH 188029; 232: Pittet y Bennett 2014. Mariscal Ramón Castilla.- 54: Bravo et al. 2016; 99: Voss y Fleck 2011; 173: Mayor et al. 2015, 2016; 174: Conga et al. 2018; 177: Voss y Fleck 2011; 198: Puertas et al. 1995. Maynas.- 15: Aquino et al. 2014a; 28: Heymann et al. 2002; 31: Heymann et al. 2002; 32: Heymann et al. 2002; 35: Heymann et al. 2002; 38: AMNH 73560-2; 59: AMNH 98470; 95: Aquino et al. 2015; 104: AMNH 73722, 73724; 107: FMNH 86933-7; 122: AMNH 98358, 98404; 150: Aquino et al. 2015; 155: Charpentier et al. 2015; 159: Aquino et al. 2007a, 2009a, 2012b, Navarro y Terrones 2006; 161: Aquino et al. 2014b; 168: Heymann y Aquino 1994. Putumayo.- 37: Bravo et al. 2016; 52: Bravo et al. 2016; 56: Montenegro y Moya 2011; 62: Montenegro y Moya 2011. Requena.- 221: Saldaña y Saldaña 2011; 230: Voss y Fleck 2011; 233: Torres-Oyarce et al. 2017; 234: Voss y Fleck 2011; 237: Soini 1986; 239: Torres-Oyarce et al. 2017; 241: Amanzo 2006; 245: Torres-Oyarce et al. 2017; 247: Bennet et al. 2001; 255: Amanzo 2006; 260: AMNH 99227-8; 261: Amanzo 2006; 274 : FMNH 64283-7; 290: Jorge y Velazco 2006; 291: Jorge y Velazco 2006; 292: Jorge y Velazco 2006. Ucayali.- 263: AMNH 76397, 76399, 76417-8, 76494; 276: AMNH 98490; 278: Aquino et al. 2005a; 280: Aquino et al. 2005a; 283: Aquino et al. 2005a; 287: Pacheco y Arias 2001.

Aotus nancymae 
Loreto.- 162: Hershkovitz 1983; 163: FMNH 122771; 165: Hershkovitz 1983; 172: FMNH 122763-6; 176: FMNH 122772-3; 179: FMNH 12276870; 205: Aquino 2005; 208: Aquino 2005; 209: Aquino 2005; 212: AMNH 187961-2; 215: Aquino 2005; 216: Aquino 2005; 218: Aquino y Bodmer 2004; 220: Aquino 2005; 222: Aquino 2005; 225: Hershkovitz 1983; 229: Allgas et al. 2018. Mariscal Ramón Castilla.- 99: Voss y Fleck 2011; 177: Voss y Fleck 2011; 198: Puertas et al. 1995. Maynas.- 96: Voss y Fleck 2011; 100: Hershkovitz 1984; 109: Glander et al. 1984; 122: FMNH 35057-35103; 145: Aquino y Encarnación 1986. Requena.- 234: Voss y Fleck 2011; 241: Amanzo 2006; 247: Bennet et al. 2001; 261: Amanzo 2006. Ucayali.- 263: AMNH 76471-5; 280: Aquino et al. 2005a; 283: Aquino et al. 2005a.

Aotus nigriceps

Requena.- 274: FMNH 64282. Ucayali.- 270: AMNH 98327-8.

\section{Aotus vociferans}

Mariscal Ramón Castilla.- 54: Aquino et al. 2016, Bravo et al. 2016. Maynas.- 6: Aquino y Encarnación 1986; 15: Aquino et al. 2014a; 19: Aquino et al. 2014a; 24: Aquino et al. 2016; 31: Heymann et al. 2002; 38: AMNH 72056-66; 41: Puertas et al. 1992; 46: Aquino y Encarnación 1986; 51: Aquino y Encarnación 1986; 66: Puertas et al. 1992; 75: Bowler et al. 2016; 78: Puertas et al. 1992; 94: FMNH 122762; 104: AMNH 73416-7, 73431; 117: FMNH 86968-9; 122: AMNH 98329, 98392, 99226, MUSM 36; 125: FMNH 86966, 86967; 127: Aquino y Encarnación 1986; 128: FMNH 122759-61; 130: Puertas et al. 1992; 146: Aquino y Encarnación 1986; 159: Aquino et al. 2012b, Navarro y Terrones 2006. Putumayo.- 42: Aquino et al. 2016; 52: Bravo et al. 2016; 53: Montenegro y Moya 2011; 56: Montenegro y Moya 2011; 76: Montenegro y Moya 2011.

\section{Saimiri boliviensis peruviensis}

Loreto.-199: AMNH 188106, 188108, 188112; 205: Aquino 2005; 207: Aquino y Calle 2003; 208: Aquino 2005; 209: Aquino 2005; 210: Aquino y Calle 2003; 212: AMNH 188072-8; 215: Aquino 2005; 216: Aquino 2005; 218: Aquino y Bodmer 2004; 220: Aquino 2005; 222: Aquino 2005; 225: Hershkovitz 1984, Pacheco y Pezo 1982; 228: FMNH 86972-6; 231: AMNH 188097, 188101, 188103; 232: Pittet y Bennett 2014. Mariscal Ramón Castilla.- 198: Puertas et al. 1995. Maynas.- 122 AMNH 98265-6, FMNH 35083. Requena: 237: Soini 1986; 247: Bennet et al. 2001; 260: Hershkovitz 1984. Ucayali.- 263: AMNH 76369-83; 280: Aquino et al. 2005a; 289: AMNH 98394.

\section{Saimiri sciureus macrodon}

Alto Amazonas.- 249: AMNH 188096. Datem del Marañón.- 58: MUSM 47304; 144: FMNH 88863-4; 193: Vermeer et al. 2013. Loreto.- 55: PérezPeña et al. 2012; 61: Pérez-Peña et al. 2016; 65: Pérez-Peña et al. 2016; 88: Aquino et al. 2015; 163: FMNH 122810, 122808-9, 122811-6; 166: FMNH 122822-3; 172: FMNH 122802-7; 176: FMNH 122817-21. Mariscal Ramón Castilla.- 54: Aquino et al. 2016, Bravo et al. 2016; 71: Puertas et al. 2017; 99: AMNH 72084-8; 149: Voss y Fleck 2011; 173: Mayor et al. 2015; 177: Voss y Fleck 2011; 198: Puertas et al. 1995. Maynas.- 3: Pérez-Peña et al. 2019; 10 Pérez-Peña et al. 2019; 12; Pérez-Peña et al. 2019; 15: Aquino et al. 2014a; 19: Aquino et al. 2014a; 24: Aquino et al. 2016; 28: Heymann et al. 2002; 31: Heymann et al. 2002; 32: Heymann et al. 2002; 35: Heymann et al. 2002; 38: AMNH 72068-82; 75: Bowler et al. 2016; 95: Aquino et al. 2015; 96: Voss y Fleck 2011; 104: AMNH 73418-30; 109: Voss y Fleck 2011, Glander et al. 1984; 110: Glander et al. 1984; 116: FMNH 125387-96; 117: FMNH 86970-1; 122: AMNH 98267-8, 98273-4, FMNH 24207, USNM 397843; 128: MUSM 24; 150: Aquino et al. 2015; 155: Charpentier et al. 2015; 159: Aquino et al. 2007a, 159: Aquino et al. 2009a, 2012b, Navarro y Terrones 2006; 161: Aquino et al. 2014b; 168: Heymann y Aquino 1994. Putumayo.- 37: Bravo et al. 2016; 42: Aquino et al. 2016; 50: Aquino et al. 2007b; 52: Bravo et al. 2016; 53: Montenegro y Moya 2011; 56: Montenegro y Moya 2011; 62; Montenegro y Moya 2011; 76: Montenegro y Moya 2011. Requena.- 230: Voss y Fleck 2011; 234: Voss y Fleck 2011, Fleck y Harder 2000; 239: TorresOyarce et al. 2017; 241: Amanzo 2006; 245:Torres-Oyarce et al. 2017; 255: Amanzo 2006; 260: Hershkovitz 1984; 261: Amanzo 2006. Ucayali.- 281: Aquino et al. 2005a; 287: Pacheco y Arias 2001; 289: Pacheco y Arias 2001.

\section{Callicebus (Plecturocebus) cupreus}

Mariscal Ramón Castilla.- 99: Voss y Fleck 2011; 149: Voss y Fleck 2011 173: Mayor et al. 2015; 177: Voss y Fleck 2011; 198: Puertas et al. 1995. Maynas.- 108: Tapia-Ruiz y Gálvez-Carrillo 2012; 109: Voss y Fleck 2011; 110: Glander et al. 1984; 122: AMNH 98381, 98388; 168: Heymann y Aquino 1994; 180: Terrones-Ruiz et al. 2004, Nadjafzadeh y Heymann 2008. Requena.224:AMNH 98373-4; 233: Torres-Oyarce et al. 2017; 234: Voss y Fleck 2011; 241: Amanzo 2006; 239: Torres-Oyarce et al. 2017; 245: Torres-Oyarce et al. 2017; 247: Bennet et al. 2001; 255: Amanzo 2006; 261: Amanzo 2006; 274: Hershkovitz 1990. Ucayali.- 263: AMNH 76419-22; 281: Aquino et al. 2005a.
Datem del Marañón.- 193: Vermeer et al. 2013. Loreto.- 61: Pérez-Peña et al. 2016; 65: Pérez-Peña et al. 2016; 88: Aquino et al. 2015; 163: FMNH 122786; 170: FMNH 122787; 172: FMNH 122783-5; 210: Aquino y Calle 2003. Maynas.- 1: AMNH 72162-3; 10: Pérez-Peña et al. 2019; 12: Pérez-Peña et al. 2019; 15: Aquino et al. 2014a; 19: Aquino et al. 2014a; 28: Heymann et al. 2002; 29: Heymann et al. 2002; 31: Heymann et al. 2002; 35: Heymann et al. 2002; 38: AMNH 72140-61; 75: Bowler et al. 2016; 95: Aquino et al. 2015; 104: AMNH 73410-5; 107: FMNH 86980-8; 122: AMNH 98377-80, FMNH 24210, MUSM 9; 150: Aquino et al. 2015; 155: Charpentier et al. 2015; 159: Aquino et al. 2007a, 2009a, 2012b, Navarro y Terrones 2006; 161 : Aquino et al. 2014b. Putumayo.- 50: Aquino et al. 2007b; 53: Montenegro y Moya 2011. Ucayali.- 287: Pacheco y Arias 2001; 289: Pacheco y Arias 2001.

Callicebus lucifer

Loreto.- 88: Aquino et al. 2015. Mariscal Ramón Castilla.- 54: Aquino et al. 2016, Bravo et al. 2016; 71: Puertas et al. 2017. Maynas.- 1: AMNH 72167; 3: Pérez-Peña et al. 2019; 24: Aquino et al. 2016; 38: AMNH 72164-6; 44: MUSM 46902-3; 59: AMNH 98366, 98375; 75: Bowler et al. 2016; 95: Aquino et al. 2015; 100: AMNH 74383-7; 107: FMNH 86978, 86979; 148: MUSM 46904-5; 150: Aquino et al. 2015; 155: Charpentier et al. 2015; 158: Aquino et al. 2008; 159: Aquino et al. 2007a, 2009a, 2012b, Navarro y Terrones 2006; 160: MUSM 50736; 161: Aquino et al. 2014b. Putumayo.- 37: Bravo et al. 2016; 42: Aquino et al. 2016; 45: Aquino et al. 2008; 50: Aquino et al. 2007b; 52: Bravo et al. 2016; 53: Montenegro y Moya 2011; 56: Montenegro y Moya 2011; 62: Montenegro y Moya 2011; 76: Montenegro y Moya 2011.

Cacajao calvus ucayalii

Mariscal Ramón Castilla.- 99: Voss y Fleck 2011; 173: Mayor et al. 2015, 2016, Mayor y Bowler 2014; 174: Conga et al. 2015; 177: Voss y Fleck 2011; 186: Bowler y Bodmer 2011, Conga et al. 2014, Mayor y Bowler 2014; 198 : Puertas et al. 1995. Maynas.- 122: AMNH 183286, 98275, 98473; 169: Aquino y Puertas 1996. Requena.- 234: Fleck y Harder 2000, Voss y Fleck 2011; 247 : Bennet et al. 2001; 275: Hershkovitz 1987; 279: AMNH 98316; 291: Jorge y Velazco 2006; 292: Jorge y Velazco 2006. Ucayali.- 263: AMNH 76390-2; 278: Aquino et al. 2005a; 280: Aquino et al. 2005a; 283: Aquino et al. 2005a.

Pithecia aequatorialis

Loreto.- 61: Pérez-Peña et al. 2016, 65: Pérez-Peña et al. 2016; 88: Aquino et al. 2015. Maynas.- 15: Aquino et al. 2014a; 28: Heymann et al. 2002; 29: Heymann et al. 2002; 95: Aquino et al. 2015; 104: AMNH 73535; 107: Hershkovitz 1987; 122: Hershkovitz 1987; 150: Aquino et al. 2015; 155: Charpentier et al. 2015; 159: Aquino et al. 2007a, 2009a, 2012b, Navarro y Terrones 2006; 161: Aquino et al. 2014b.

Pithecia isabela

Loreto.- 199: AMNH 187983-4; 205: Aquino 2005; 207: Aquino y Calle 2003; 208: Aquino 2005; 209: Aquino 2005; 210: Aquino y Calle 2003; 212 : 187974-82; 215: Aquino 2005; 216: Aquino 2005; 218: Aquino y Bodmer 2004; 220: Aquino 2005; 222: Aquino 2005; 225: Hershkovitz 1987, Marsh 2014, Pacheco y Pezo 1982; 226: Marsh 2014; 228: Marsh 2014; 229: Allgas et al. 2018; 232: Pittet y Bennett 2014. Requena.- 237: Soini 1986.

Pithecia monachus

Mariscal Ramón Castilla.- 54: Aquino et al. 2016, Bravo et al. 2016; 71: Puertas et al. 2017; 99: Voss y Fleck 2011; 149: Voss y Fleck 2011; 173: Mayor et al. 2015; 177: Voss y Fleck 2011; 198: Puertas et al. 1995. Maynas.- 1: AMNH 71816-7; 3: Pérez-Peña et al. 2019; 24: Aquino et al. 2016; 28: Heymann et al. 2002; 75: Bowler et al. 2016; 104: AMNH 73536-9; 107: Hershkovitz 1987; 109: Voss y Fleck 2011; 168: Heymann y Aquino 1994. Putumayo.- 37: Bravo et al. 2016; 42: Aquino et al. 2016; 50: Aquino et al. 2007b; 52: Bravo et al. 2016; 53: Montenegro y Moya 2011; 56: Montenegro y Moya 2011; 62: Montenegro y Moya 2011; 76: Montenegro y Moya 2011. Requena.- 233: Bardales-Alvites et al. 2017, Torres-Oyarce et al. 2017; 234: Fleck et al. 1999, Voss y Fleck 2011; 239: Torres-Oyarce et al. 2017; 241: Amanzo 2006; 245: Torres-Oyarce et al. 2017; 247: Bennet et al. 2001; 255: Amanzo 2006; 260 : AMNH 98480, 99212; 261: Amanzo 2006; 274: FMNH 64270-1. Ucayali.- 263: AMNH 76409-14; 278: Aquino et al. 2005a; 280: Aquino et al. 2005a; 283: Aquino et al. 2005a; 287: Pacheco y Arias 2001; 289: Pacheco y Arias 2001. Pithecia napensis

Alto Amazonas.- 246: Patterson y López-Wong 2014; 256: Patterson y LópezWong 2014. Datem del Marañón.- 184: AMNH 98346. Loreto.- 88: Aquino et al. 2015; 195: AMNH 187989. Maynas.- 10: Pérez-Peña et al. 2019; 12: Pérez-Peña et al. 2019; 19: Aquino et al. 2014a; 38: AMNH 71802-12; 95: Aquino et al. 2015; 150: Aquino et al. 2015.

\section{Alouatta seniculus}

Alto Amazonas.- 256: Patterson y López-Wong 2014. Datem del Marañón.193: Vermeer et al. 2013. Loreto.- 61: Pérez-Peña et al. 2016; 65: Pérez-Peña et al. 2016; 88: Aquino et al. 2015; 205: Aquino 2005; 207: Aquino y Calle 
2003; 208: Aquino 2005; 209: Aquino 2005; 210: Aquino y Calle 2003; 212 : AMNH 187991-8006; 215: Aquino 2005; 216: Aquino 2005; 218: Aquino y Bodmer 2004; 220: Aquino 2005; 222: Aquino 2005; 225: Pacheco y Pezo 1982; 228: FMNH 86943-50; 229: Allgas et al. 2018; 232: Pittet y Bennett 2014. Mariscal Ramón Castilla.- 54: Aquino et al. 2016, Bravo et al. 2016; 71: Puertas et al. 2017; 173: Mayor et al. 2015; 198: Puertas et al. 1995. Maynas.- 1: AMNH 71751-2; 3: Pérez-Peña et al. 2019, 12: Pérez-Peña et al. 2019; 24: Aquino et al. 2016; 28: Heymann et al. 2002; 31: Heymann et al. 2002; 38: AMNH 71743-50; 59: AMNH 98466; 67: Gilmore 2005; 75: Bowler et al. 2016; 90: Voss y Fleck 2011; 95: Aquino et al. 2015; 96: Voss y Fleck 2011; 104: AMNH 73545-53; 122: AMNH 98315, 98474, 99239, 99240; 150: Aquino et al. 2015; 159: Aquino et al. 2007a, 2009a, 2012b, Navarro y Terrones 2006; 161: Aquino et al. 2014b. Putumayo.- 37: Bravo et al. 2016; 42: Aquino et al. 2016; 50: Aquino et al. 2007b; 52: Bravo et al. 2016; 53: Montenegro y Moya 2011; 56: Montenegro y Moya 2011; 62: Montenegro y Moya 2011; 76: Montenegro y Moya 2011. Requena.- 221: Saldaña y Saldaña 2011; 233: Bardales-Alvites et al. 2017; 234: Fleck y Harder 2000, Voss y Fleck 2011; 236: Soini 1992; 237: Soini 1986; 239: Torres-Oyarce et al. 2017; 241: Amanzo 2006; 245: Torres-Oyarce et al. 2017; 247: Bennet et al. 2001; 261: Amanzo 2006; 262: UMMZ 62507; 274: FMNH 64278-81; 291: Jorge y Velazco 2006. Ucayali.- 263: AMNH 76384-8; 278: Aquino et al. 2005a; 280: Aquino et al. 2005a; 282: AMNH 98319; 283: Aquino et al. 2005a; 287: Pacheco y Arias 2001; 289: Pacheco y Arias 2001; 284: AMNH 99234-5.

\section{Ateles belzebuth}

Datem del Marañón.- 86: Aquino et al. 2013; 156: Aquino et al. 2013; 193: Vermeer et al. 2013. Loreto.- 61: Pérez-Peña et al. 2016; 65: Pérez-Peña et al. 2016; 195: AMNH 188124-32; 205: Aquino 2005; 207: Aquino y Calle 2003; 208: Aquino 2005; 209: Aquino 2005; 215: Aquino 2005; 216: Aquino 2005; 218: Aquino y Bodmer 2004, Aquino y Bodmer 2006; 220: Aquino 2005; 222: Aquino 2005; 229: Allgas et al. 2018. Mariscal Ramón Castilla-- 99: Voss y Fleck 2011. Maynas.- 10:Pérez-Peña et al. 2019; 12: Pérez-Peña et al. 2019; 14: Aquino et al. 2013; 15: Aquino et al. 2014a; 17: Aquino et al. 2013; 19: Aquino et al. 2014a; 21: Aquino et al. 2013; 23: Aquino et al. 2013; 38: AMNH 71785-94; 104: AMNH 73540-4; 122: AMNH 98401-4, MUSM 1894. Requena.- 230: Voss y Fleck 2011; 234: Voss y Fleck 2011.

\section{Ateles chamek}

Datem del Marañón.- 144: FMNH 88835-8; 193: Vermeer et al. 2013. Loreto205: Aquino 2005; 208: Aquino 2005; 209: Aquino 2005; 215: Aquino 2005 216: Aquino 2005; 218: Aquino y Bodmer 2004, Aquino y Bodmer 2006; 220 : Aquino 2005; 222: Aquino 2005. Mariscal Ramón Castilla.- 173: Mayor et al. 2015; 198: Puertas et al. 1995. Maynas.- 122: AMNH 98340. Requena.- 233: Bardales-Alvites et al. 2017, Torres-Oyarce et al. 2017; 234: Fleck y Harder 2000; 241: Amanzo 2006; 245: Torres-Oyarce et al. 2017; 255: Amanzo 2006; 261: Amanzo 2006; 274: FMNH 64273-6; 290: Jorge y Velazco 2006; 291: Jorge y Velazco 2006; 292: Jorge y Velazco 2006. Ucayali.- 263: AMNH 76389; 278: Aquino et al. 2005a; 280: Aquino et al. 2005a; 283: Aquino et al. 2005a; 287: Pacheco y Arias 2001; 289: Pacheco y Arias 2001.

Lagothrix flavicauda

Alto Amazonas.- 256: Patterson y López-Wong 2014.

Lagothrix lagothricha lagothricha

Mariscal Ramón Castilla.- 54: Aquino et al. 2016, Bravo et al. 2016; 71: Puertas et al. 2017. Maynas.- 1: AMNH 71773-5; 3: Pérez-Peña et al. 2019; 24: Aquino et al. 2016; 38: AMNH 71757-60; 75: Bowler et al. 2016; 100: AMNH 74422-7. Putumayo.- 37: Bravo et al. 2016; 42: Aquino et al. 2016; 50: Aquino et al. 2007b; 52: Bravo et al. 2016; 53: Montenegro y Moya 2011; 56: Montenegro y Moya 2011; 62: Montenegro y Moya 2011; 76: Montenegro y Moya 2011.

Lagothrix lagothricha poeppigii

Alto Amazonas.- 254: FMNH 20130. Datem del Marañón.- 72: MUSM 37709; 114: FMNH 88826. Loreto.- 55: Pérez-Peña et al. 2012; 61: Pérez-Peña et al. 2016; 65: Pérez-Peña et al. 2016; 80: MUSM 17557; 88: Aquino et al. 2015; 195: AMNH 188162; 199: AMNH 188150, 188153-6; 205: Aquino 2005; 207 : Aquino y Calle 2003; 208: Aquino 2005; 209: Aquino 2005; 210: Aquino y Calle 2003; 212: AMNH 188142-9; 215: Aquino 2005; 216: Aquino 2005; 218: Aquino y Bodmer 2004; 220: Aquino 2005; 222: Aquino 2005; 225: FMNH 122790; 228: FMNH 86928, 86940-2; 229: Allgas et al. 2018; 232: Pittet y Bennett 2014. Mariscal Ramón Castilla.- 173: Mayor et al. 2015, 2016; 177: Voss y Fleck 2011; 198: Puertas et al. 1995. Maynas.- 10: Pérez-Peña et al. 2019; 12: Pérez-Peña et al. 2019; 15: Aquino et al. 2014a; 19: Aquino et al. 2014a; 31: Heymann et al. 2002; 32: Heymann et al. 2002; 38: AMNH 71761, 71763-70; 59: AMNH 31097, 98467; 95: Aquino et al. 2015; 104: AMNH 73554-8; 107: FMNH 86925-7; 122: AMNH 98332, 98822, MUSM 1893, 1904; 150: Aquino et al. 2015; 155: Charpentier et al. 2015; 159: Aquino et al. 2007a, 2009a, 2012b, Navarro y Terrones 2006; 161: Aquino et al. 2014b;
168: Heymann y Aquino 1994. Requena.- 221: Saldaña y Saldaña 2011; 230: Voss y Fleck 2011; 233: Bardales-Alvites et al. 2017, Torres-Oyarce et al. 2017; 234: Voss y Fleck 2011; 237: Soini 1986; 239: Torres-Oyarce et al. 2017; 241: Amanzo 2006; 245: Torres-Oyarce et al. 2017; 247: Bennet et al. 2001; 255: Amanzo 2006; 261: Amanzo 2006; 272: MUSM 1859-60; 274: FMNH 64272; 290: Jorge y Velazco 2006; 291: Jorge y Velazco 2006; 292: Jorge y Velazco 2006. Ucayali.- 263: AMNH 75291, 76393-6; 264: MUSM 687-8; 278: Aquino et al. 2005a; 280: Aquino et al. 2005a; 283: Aquino et al. 2005a; 287: Pacheco y Arias 2001; 289: Pacheco y Arias 2001.

Coendou bicolor

Loreto.- 55: Pérez-Peña et al. 2012. Maynas.- 159: Aquino et al. 2012b, Navarro y Terrones 2006.

Coendou prehenselis

Loreto.- 33: MUSM 34603; 225: FMNH 122987. Maynas.- 38: Voss 2011; 67 Gilmore 2005; 74: Griffiths et al. 2020; 75: Bowler et al. 2016; 83: Voss 2011; 103: Voss 2011; 108: Voss 2011; 122: AMNH 98578; 132: Hice y Velazco 2012; 152: MUSM 34604. Requena.- 234: Voss 2011.

Dinomys branickii

Maynas.- 122: Patton 2015. Requena.- 234: MUSM 11231.

Hydrochoerus hydrochaeris

Datem del Marañón.- 187: MVZ63516. Loreto.- 55: Pérez-Peña et al. 2012; 201: KU158245; 225: FMNH 122988; 227: FMNH 86923-4. Mariscal Ramón Castilla.- 54: Bravo et al. 2016. Maynas.- 38: AMNH 71726-7, 71840; 122: AMNH 98631-4, 98881-2; 159: Aquino et al. 2007a; 169: Navarro y Terrones 2006. Putumayo.- 50: Aquino et al. 2007b; 56: Montenegro y Moya 2011; 62: Montenegro y Moya 2011. Requena.- 221: Saldaña y Saldaña 2011; 230: FMNH 88900; 232: MUSM 38512; 234: AMNH 268264, MUSM 11226-30; 261: Amanzo 2006.

\section{Dasyprocta fuliginosa}

Alto Amazonas.- 157: MUSM 16413; 246: Patterson y López-Wong 2014; 248: Patterson y López-Wong 2014; 253: Osgood 1914; 256: Patterson y López-Wong 2014. Loreto.- 55: Falcón-Ayapi et al. 2019, Pérez-Peña et al. 2012; 207: Aquino y Calle 2003; 208: Aquino 2005; 209: Aquino y Puertas 1996; 210: Aquino y Calle 2003; 216: Aquino 2005; 222: Aquino 2005; 227: FMNH 86921; 232: Pittet y Bennett 2014. Mariscal Ramón Castilla.- 54: Bravo et al. 2016; 71: Puertas et al. 2017; 173: Mayor et al. 2015; 175: FMNH 88903-6; 190: Aquino y Puertas 1996. Maynas.- 1: AMNH 71826, 71828-31; 3: Pérez-Peña et al. 2019; 10: Pérez-Peña et al. 2019; 12: PérezPeña et al. 2019; 38: AMNH 71825, 71827, 71832-3; 40: Lönnberg 1921; 59: AMNH 98238; 67: Gilmore 2005; 69: LSUMZ 28398; 98: LSUMZ 28005-6; 104: AMNH 73580-5; 122: Thomas 1928; 131: MUSM 34081-3; 141: Hice y Velazco 2012; 159: Aquino et al. 2007a, 2012b, Navarro y Terrones 2006; 169: Aquino y Puertas 1996; 205: Aquino 2005. Putumayo.- 37: Bravo et al. 2016; 50: Aquino et al. 2007b; 52: Bravo et al. 2016; 56: Montenegro y Moya 2011; 62: Montenegro y Moya 2011; 76: Montenegro y Moya 2011. Requena.- 221: Saldaña y Saldaña 2011; 230: FMNH 88901-2; 233: BardalesAlvites et al. 2017, Torres-Oyarce et al. 2017; 234: Fleck y Harder 2000; 239: Bardales-Alvites et al. 2017, Torres-Oyarce et al. 2017; 241: Amanzo 2006; 245: Torres-Oyarce et al. 2017; 255: Amanzo 2006; 261: Amanzo 2006; 290 Jorge y Velazco 2006; 291: Jorge y Velazco 2006. Ucayali.- 263: AMNH 75292, 76424-6; 289: Pacheco y Arias 2001.

\section{Myoprocta pratti}

Datem del Marañón.- 143: Teta 2019. Loreto.- 49: KU158218-9; 55: FalcónAyapi et al. 2019; 201: KU158217; 71: Puertas et al. 2017; 99: Teta 2019; 153: FMNH 88908; 175: Teta 2019. Maynas.- 3: Pérez-Peña et al. 2019; 10: Pérez-Peña et al. 2019; 38: Teta 2019; 47: MUSM 24441; 64: LSUMZ 28411; 83: Teta 2019; 90: Teta 2019; 93: LSUMZ 28400; 101: LSUMZ 28412, 28418 ; 103: AMNH 98240; 104: Teta 2019; 108: Teta 2019; 122: AMNH 98815, FMNH 24793-4; 159: Aquino et al. 2012b, Navarro y Terrones 2006; 167: FMNH 30465. Putumayo.- 37: Bravo et al. 2016; 50: Aquino et al. 2007b; 52: Bravo et al. 2016; 56: Montenegro y Moya 2011; 76: Montenegro y Moya 2011. Requena.- 219: MUSM 23824; 230: Teta 2019; 233: Torres-Oyarce et al. 2017; 234: AMNH 268267-8, MUSM 11234-7; 274: FMNH 64295. Ucayali.- 263: Teta 2019; 269: MUSM 693; 289: Pacheco y Arias 2001.

Cuniculus paca

Alto Amazonas.- 246: Patterson y López-Wong 2014; 248: Patterson y LópezWong 2014; 256: Patterson y López-Wong 2014. Datem del Marañón.- 143: FMNH 88895-7; 214: FMNH 88898. Loreto.- 49: KU 158166, 158256; 55: Falcón-Ayapi et al. 2019, Pérez-Peña et al. 2012; 79: MUSM 17727; 201: KU 158165; 205: Aquino 2005; 208: Aquino 2005; 216: Aquino 2005; 222: Aquino 2005; 225: FMNH 122989; 232: Pittet y Bennett 2014. Mariscal Ramón Castilla.-54: Bravo et al. 2016; 71: Puertas et al. 2017; 173: Mayor et al. 2015, 2016; 175: FMNH 88899. Maynas.- 3: Pérez-Peña et al. 2019; 10: 
Pérez-Peña et al. 2019; 12: Pérez-Peña et al. 2019; 38: AMNH 71836-9; 67: Gilmore 2005; 98: LSUMZ 28003; 104: AMNH 73578-9; 108: FMNH 86922; 122: AMNH 98556; 141: Hice y Velazco 2012; 154: MUSM 34080; 159 Aquino et al. 2007a, 2009b, 2012a, 2012b, Navarro y Terrones 2006 ; 169: MUSM 2650-3. Putumayo.- 37: Bravo et al. 2016; 52: Bravo et al. 2016; 53: Montenegro y Moya 2011; 56: Montenegro y Moya 2011; 62: Montenegro y Moya 2011; 76: Montenegro y Moya 2011. Requena.- 221: Saldaña y Saldaña 2011; 233: Bardales-Alvites et al. 2017; 234: Fleck y Harder 2000 239: Bardales-Alvites et al. 2017; 241: Amanzo 2006; 255: Amanzo 2006; 261: Amanzo 2006; 290: Jorge y Velazco 2006; 291: Jorge y Velazco 2006; 292: Jorge y Velazco 2006. Ucayali.- 264: MUSM 689; 269: MUSM 18036; 289: Pacheco y Arias 2001.

\section{Leopardus pardalis}

Alto Amazonas.- 248: Patterson y López-Wong 2014. Datem del Marañón.144: FMNH 88886; 187: AMNH 98607. Loreto.- 55: Falcón-Ayapi et al. 2019, Pérez-Peña et al. 2012; 207: Aquino y Calle 2003; 225: FMNH 123026; 228: FMNH 86901; 232: Pittet y Bennett 2014; Mariscal Ramón Castilla; 99: Voss y Fleck 2017; 177: Voss y Fleck 2017. Maynas.- 3: Pérez-Peña et al. 2019; 5: MUSM 9392; 10: Pérez-Peña et al. 2019; 12: Pérez-Peña et al. 2019; 18: Kolowski y Alonso 2010; 74: Griffiths et al. 2020; 97: AMNH 98598; 122 AMNH 146842, 98595-7, 98599-608, MUSM 5992; 159: Aquino et al. 2007a, 2012b, Navarro y Terrones 2006. Putumayo.- 37: Bravo et al. 2016; 50: Aquino et al. 2007b; 52: Bravo et al. 2016; 62: Montenegro y Moya 2011. Requena.- 230: Voss y Fleck 2017; 234: Voss y Fleck 2017; 261: Amanzo 2006; 292: Jorge y Velazco 2006. Ucayali.- 263: FMNH 25351; 268: MUSM 685; 289: Pacheco y Arias 2001

Leopardus wiedii

Alto Amazonas.- 246: Patterson y López-Wong 2014; 253: FMNH 19646. Loreto.- 55: Falcón-Ayapi et al. 2019. Maynas.- 10: Pérez-Peña et al. 2019; 100 AMNH 74428; 159: Navarro y Terrones 2006. Putumayo.- 76: Montenegro y Moya 2011. Requena.- 230: Voss y Fleck 2017.

Puma concolor

Alto Amazonas.- 248: Patterson y López-Wong 2014. Loreto.- 55: Falcón-Ayapi et al. 2019, Pérez-Peña et al. 2012; 211: AMNH 99193; 232: Pittet y Bennett 2014. Mariscal Ramón Castilla.- 54: Bravo et al. 2016. Maynas.- 12: PérezPeña et al. 2019; 122: AMNH 98669, 99190-5; 159: Aquino et al. 2012b, Navarro y Terrones 2006. Putumayo.- 52: Bravo et al. 2016; 56: Montenegro y Moya 2011. Requena.- 234: MUSM 20964; 255: Amanzo 2006. Ucayali.268: MUSM 684; 289: AMNH 98670.

\section{Puma yagouaroundi}

Alto Amazonas.- 257: MUSM 419. Loreto.- 55: Falcón-Ayapi et al. 2019. Maynas.- 122: AMNH 98553-5; 126: USNM 273937; 159: Navarro y Terrones 2006. Putumayo.- 52: Bravo et al. 2016. Requena.- 234: MUSM 20965. Ucayali.- 289: AMNH 98649.

\section{Panthera onca}

Alto Amazonas.- 246: Patterson y López-Wong 2014; 248: Patterson y LópezWong 2014; 256: Patterson y López-Wong 2014. Loreto.- 55: Falcón-Ayapi et al. 2019, Pérez-Peña et al. 2012; 205: Aquino 2005; 208: Aquino 2005; 211 : AMNH 99167; 216: Aquino 2005; 222: Aquino 2005; 232: Pittet y Bennett 2014. Maynas.- 3: Pérez-Peña et al. 2019; 97: AMNH 98673, 99168; 107: FMNH 86902-3; 119: AMNH 98672; 122: AMNH 98674-5, 98677-8, FMNH 34691; 159: Aquino et al. 2012b, Navarro y Terrones 2006. Putumayo.- 37: Bravo et al. 2016; 50: Aquino et al. 2007b; 52: Bravo et al. 2016; 56: Montenegro y Moya 2011; 62: Montenegro y Moya 2011; 76: Montenegro y Moya 2011. Requena.- 234: MUSM 20963; 241: Amanzo 2006; 255: Amanzo 2006; 261: Amanzo 2006; 290: Jorge y Velazco 2006; 291: Jorge y Velazco 2006; 292: Jorge y Velazco 2006. Ucayali.- 263: AMNH 76451; 265: MUSM 683; 266: MUSM 5740; 288: FMNH 57199; 289: Pacheco y Arias 2001.

\section{Atelocynus microtis}

Loreto.- 55.- Falcón-Ayapi et al. 2019; 197: USNM 273936; 205: Aquino 2005; 208: Aquino 2005; 216: Aquino 2005; 222: Aquino 2005; 232: Pittet y Bennett 2014. Maynas.- 3: Pérez-Peña et al. 2019; 10: Pérez-Peña et al. 2019; 12: Pérez-Peña et al. 2019; 122: AMNH 98565, 98639, 99260, 99303. Putumayo.- 37: Bravo et al. 2016; 52: Bravo et al. 2016. Ucayali.- 286: MUSM 404.

\section{Speothos venaticus}

Loreto.- 209: Aquino y Puertas 1996. Mariscal Ramón Castilla.- 190: Aquino y Puertas 1996. Maynas.- 94: Aquino y Puertas 1996; 122: AMNH 98558-61; FMNH 146313, 146315; 159: Aquino et al. 2012b; 169: Aquino y Puertas 1996. Putumayo.- 37: Bravo et al. 2016. Requena.- 241: Amanzo 2006. Ucayali: 289: Pacheco y Arias 2001.

Tremarctos ornatus

Ucayali.- 287: Pacheco y Arias 2001

\section{Lontra longicaudis}

Loreto.- 55: Pérez-Peña et al. 2012. Maynas.- 38: AMNH 71860; 122: AMNH 98588, MUSM 5999; 142: MUSM 16461; 159: Aquino et al. 2012b; Navarro y Terrones 2006. Putumayo.- 37: Bravo et al. 2016; 53: Montenegro y Moya 2011; 62: Montenegro y Moya 2011. Requena.- 234: Voss y Fleck 2017; 241: Amanzo 2006; 291: Jorge y Velazco 2006.

Pteronura brasiliensis

Loreto.- 55: Pérez-Peña et al. 2012. Mariscal Ramón Castilla.- 54: Bravo et al. 2016. Maynas.- 12: Pérez-Peña et al. 2019; 38: AMNH 73587; 97: AMNH 98590, 98594, FMNH 146402; 100: Voss y Fleck 2017; 122: AMNH 98591-3, 99178-80, FMNH 146403, 34699, 34700. Putumayo.- 37: Bravo et al. 2016; 52: Bravo et al. 2016; 53: Montenegro y Moya 2011; 56: Montenegro y Moya 2011; 76: Montenegro y Moya 2011. Requena.- 224: AMNH 99181; 234: Voss y Fleck 2017.

Eira barbara

Datem del Marañón.- 144: FMNH 88882-5. Loreto.- 55: Falcón-Ayapi et al. 2019; 201: KU 158253; 205: Aquino 2005; 207: Aquino y Calle 2003; 208: Aquino 2005; 210: Aquino y Calle 2003; 216: Aquino 2005; 222: Aquino 2005; 228: FMNH 86914. Mariscal Ramón Castilla.- 54: Bravo et al. 2016; 99: Voss y Fleck 2017. Maynas.- 12: Pérez-Peña et al. 2019; 38: AMNH 71850-2; 75: Bowler et al. 2016; 100: AMNH 74416-8; 122: AMNH 98582, 98585-6; 142: MUSM 16459; 155: Charpentier et al. 2015; 159: Aquino et al. 2007a, 2012b, Navarro y Terrones 2006. Putumayo.- 37: Bravo et al. 2016; 50: Aquino et al. 2007b; 52: Bravo et al. 2016; 56: Montenegro y Moya 2011; 62: Montenegro y Moya 2011. Requena.- 233: Torres-Oyarce et al. 2017; 234: Voss y Fleck 2017; 239: Torres-Oyarce et al. 2017; 241: Amanzo 2006; 261: Amanzo 2006; 245: Torres-Oyarce et al. 2017; 290: Jorge y Velazco 2006; 291: Jorge y Velazco 2006. Ucayali.- 263: AMNH 76489-91; 287: Pacheco y Arias 2001; 289: Pacheco y Arias 2001.

Galictis vittata

Loreto.- 55: Pérez-Peña et al. 2012. Maynas.- 122: AMNH 98568-9, 985712; 142: MUSM 16460; 159: Aquino et al. 2012b, Navarro y Terrones 2006. Requena.- 234: Voss y Fleck 2017.

Bassaricyon allen

Mariscal Ramón Castilla.- 54: Bravo et al. 2016. Maynas.- 41: Puertas et al. 1992; 66: Puertas et al. 1992; 75: Bowler et al. 2016; 78: Puertas et al. 1992; 107: FMNH 86909; 117: FMNH 86908; 122: AMNH 98653-4, 98662; 130: Puertas et al. 1992; 159: Aquino et al. 2012b. Putumayo.- 37: Bravo et al. 2016. Requena.- 234: Voss y Fleck 2017; 272: MUSM 881. Ucayali.- 268: MUSM 696; 289: AMNH 98709.

Nasua nasua

Alto Amazonas.- 256: Patterson y López-Wong 2014. Datem del Marañón.144: FMNH 88875. Loreto.- 55: Falcón-Ayapi et al. 2019, Pérez-Peña et al. 2012; 205: Aquino 2005; 207: Aquino y Calle 2003; 208: Aquino 2005; 209 : Aquino y Puertas 1996; 210: Aquino y Calle 2003; 216: Aquino 2005; 222: Aquino 2005; 225: Pacheco y Pezo 1982; 228: FMNH 86910-13; 231: AMNH 188204. Mariscal Ramón Castilla.- 173: Mayor et al. 2015, 2016; 177: Voss y Fleck 2017; 190: Aquino y Puertas 1996. Maynas.- 3: Pérez-Peña et al. 2019; 10: Pérez-Peña et al. 2019; 12: Pérez-Peña et al. 2019; 38: AMNH 71841-9, 71854; 100: AMNH 74419-21; 104: AMNH 73764; 122: AMNH 98499-502, 98557-8, FMNH 34685; 159: Aquino et al. 2007a, 2012b, Navarro y Terrones 2006; 169: Aquino y Puertas 1996. Putumayo.- 37: Bravo et al. 2016; 50: Aquino et al. 2007b; 52: Bravo et al. 2016; 53: Montenegro y Moya 2011; 56: Montenegro y Moya 2011; 76: Montenegro y Moya 2011. Requena.- 221: Saldaña y Saldaña 2011; 230: Voss y Fleck 2017; 234: Voss y Fleck 2017; 239: Torres-Oyarce et al. 2017; 241: Amanzo 2006; 245: Torres-Oyarce et al. 2017; 255: Amanzo 2006; 260: AMNH 98497, 99256; 291: Jorge y Velazco 2006. Ucayali.- 263: AMNH 76400; 288: AMNH 98493; 289: Pacheco y Arias 2001.

Potos flavus

Loreto.- 49: KU 158254-5; 205: Aquino 2005; 208: Aquino 2005; 209 : Aquino 2005; 211: AMNH 99200; 215: Aquino 2005; 216: Aquino 2005; 220 : Aquino 2005; 222: Aquino 2005; 225: FMNH 123023-4; 228: FMNH 86907; 231: AMNH 188207. Mariscal Ramón Castilla.- 99: Voss y Fleck 2017. Maynas.- 38: AMNH 71853; 41: Puertas et al. 1992; 66: Puertas et al. 1992; 75: Bowler et al. 2016; 78: Puertas et al. 1992; 107: FMNH 86905-6; 117: FMNH 86904; 130: Puertas et al. 1992; 159: Aquino et al. 2012b, Navarro y Terrones 2006; 142: MUSM 16462-2; 155: Charpentier et al. 2015; 159: Aquino et al. 2007a. Putumayo.- 37: Bravo et al. 2016; 52: Bravo et al. 2016; 56: Montenegro y Moya 2011; 62: Montenegro y Moya 2011. Requena.- 234: Voss y Fleck 2017; 241: Amanzo 2006; 255: Amanzo 2006; 261: Amanzo 2006; 274: FMNH 64290; 291: Jorge y Velazco 2006; 292: Jorge y Velazco 2006. Ucayali.- 269: MUSM 18034; 289: Pacheco y Arias 2001.

Procyon cancrivorus 
Alto Amazonas.- 246: Patterson y López-Wong 2014. Loreto.- 205: Aquino 2005; 207: Aquino y Calle 2003; 208: Aquino 2005; 216: Aquino 2005; 222: Aquino 2005. Maynas.- 10: Pérez-Peña et al. 2019; 122: AMNH 98564, 98566, 98641; 128: FMNH 123025; 159: Aquino et al. 2007a, Navarro y Terrones 2006. Putumayo.- 37: Bravo et al. 2016; 52: Bravo et al. 2016; Requena.- 230: Voss y Fleck 2017; 234: MUSM 20966

Tapirus terrestris

Alto Amazonas.- 246: Patterson y López-Wong 2014; 248: Patterson y LópezWong 2014; 256: Patterson y López-Wong 2014. Datem del Marañón.- 114 FMNH 88793; 144: FMNH 88792. Loreto.- 55: Falcón-Ayapi et al. 2019, PérezPeña et al. 2012; 87: Aquino et al. 2014c; 205: Aquino 2005; 207: Aquino y Calle 2003; 208: Aquino 2005; 212: AMNH 235208; 216: Aquino 2005; 222: Aquino 2005; 232: Pittet y Bennett 2014. Mariscal Ramón Castilla.- 54: Bravo et al. 2016; 71: Puertas et al. 2017; 99: Voss y Fleck 2017; 173: Mayor et al. 2015, 2016. Maynas.- 3: Pérez-Peña et al. 2019; 10: Pérez-Peña et al. 2019; 12: Pérez-Peña et al. 2019; 20: Aquino et al. 2014c; 38: AMNH 71728-31; 67: Gilmore 2005; 104: AMNH 73595-6; 159: Aquino et al. 2007a, 2012b, Navarro y Terrones 2006; 169: MUSM 2702-3. Putumayo.- 37: Bravo et al. 2016; 50: Aquino et al. 2007b; 52: Bravo et al. 2016; 53: Montenegro y Moya 2011; 56: Montenegro y Moya 2011; 62: Montenegro y Moya 2011; 76: Montenegro y Moya 2011. Requena.- 221: Saldaña y Saldaña 2011; 230: Voss y Fleck 2017; 233: Bardales-Alvites et al. 2017, Torres-Oyarce et al. 2017; 234: Fleck y Harder 2000, Voss y Fleck 2017; 239: Torres-Oyarce et al. 2017; 241: Amanzo 2006; 245: Torres-Oyarce et al. 2017; 255: Amanzo 2006; 261: Amanzo 2006; 290: Jorge y Velazco 2006; 291: Jorge y Velazco 2006; 292: Jorge y Velazco 2006. Ucayali.- 263: AMNH 75328, 76452; 269: MUSM 18037; 287: Pacheco y Arias 2001; 289: Pacheco y Arias 2001.

Dicotyles tajacu

Datem del Marañón.- 129: Pérez-Peña et al. 2017; 139: Pérez-Peña et al. 2017; 144: FMNH 89175. Loreto.- 48: Pérez-Peña et al. 2017; 55: Falcón-Ayapi et al. 2019, Pérez-Peña et al. 2012; 63: Pérez-Peña et al. 2017; 87: Aquino et al. 2014c; 89: Pérez-Peña et al. 2017; 217: Pérez-Peña et al. 2017; 225: FMNH 123028; 228: FMNH 86897; 232: Pittet y Bennett 2014. Mariscal Ramón Castilla.- 54: Bravo et al. 2016; 70: Pérez-Peña et al. 2017; 71: Puertas et al. 2017; 77: Pérez-Peña et al. 2017; 99: AMNH 73767; 173: Mayor et al. 2015, 2016; 177: Voss y Fleck 2017; 181: Pérez-Peña et al. 2017; 183: Pérez-Peña et al. 2017. Maynas.- 1: AMNH 71732-5; 3: Pérez-Peña et al. 2019; 10: Pérez-Peña et al. 2019; 12: Pérez-Peña et al. 2019; 20: Aquino et al. 2014c; 21: Pérez-Peña et al. 2017; 24: Pérez-Peña et al. 2017; 38: AMNH 71718-21; 67: Gilmore 2005; 75: Pérez-Peña et al. 2017; 94: Pérez-Peña et al. 2017; 104: AMNH 73589-93; 126: Pérez-Peña et al. 2017; 154: MUSM 30845; 159: Aquino et al. 2007a, 2012b, Navarro y Terrones 2006; 169: MUSM 2712-4; 171: Pérez-Peña et al. 2017; 182: Pérez-Peña et al. 2017. Putumayo.- 2: Pérez-Peña et al. 2017; 7: Pérez-Peña et al. 2017; 8: Pérez-Peña et al. 2017; 37: Bravo et al. 2016; 43: Pérez-Peña et al. 2017; 50: Aquino et al. 2007b; 52: Bravo et al. 2016; 53: Montenegro y Moya 2011; 56: Montenegro y Moya 2011; 62: Montenegro y Moya 2011; 76: Montenegro y Moya 2011. Requena.- 221: Saldaña y Saldaña 2011; 230: Voss y Fleck 2017; 233: Bardales-Alvites et al. 2017, Torres-Oyarce et al. 2017; 234: Fleck y Harder 2000, Voss y Fleck 2017; 235: Pérez-Peña et al. 2017; 238: Pérez-Peña et al. 2017; 239: Bardales-Alvites et al. 2017, Torres-Oyarce et al. 2017; 241: Amanzo 2006; 242: AMNH 98613; 243: Pérez-Peña et al. 2017; 245: TorresOyarce et al. 2017; 255: Amanzo 2006; 261: Amanzo 2006; 290: Jorge y Velazco 2006; 291: Jorge y Velazco 2006. Ucayali.- 263: AMNH 76441; 264: MUSM 692; 269: MUSM 18035; 285: Pérez-Peña et al. 2017; 287: Pacheco y Arias 2001; 289: Pacheco y Arias 2001.

\section{Tayassu pecari}

Alto Amazonas.- 256: Patterson y López-Wong 2014. Datem del Marañón.139: Pérez-Peña et al. 2017. Loreto.- 48: Pérez-Peña et al. 2017; 55: FalcónAyapi et al. 2019, Pérez-Peña et al. 2012; 63: Pérez-Peña et al. 2017; 170: FMNH 123027; 207: Aquino y Calle 2003; 208: Aquino 2005; 212: AMNH 188222; 216: Aquino 2005; 222: Aquino 2005. Mariscal Ramón Castilla; 54:
Bravo et al. 2016; 71: Puertas et al. 2017; 173: Mayor et al. 2015, 2016; 181: Pérez-Peña et al. 2017. Maynas.- 3: Pérez-Peña et al. 2019; 10: Pérez-Peña et al. 2019; 12: Pérez-Peña et al. 2019; 20: Aquino et al. 2014c; 21: PérezPeña et al. 2017; 24: Pérez-Peña et al. 2017; 38: AMNH 71722-4, 71736-8, 67: Gilmore 2005; 75: Pérez-Peña et al. 2017; 100: AMNH 74432-7; 122: AMNH 98614; 159: Aquino et al. 2012b, Navarro y Terrones 2006; 169: MUSM 2706-7; 171: Pérez-Peña et al. 2017; 182: Pérez-Peña et al. 2017; 205: Aquino 2005. Putumayo.- 2: Pérez-Peña et al. 2017; 8: Pérez-Peña et al. 2017; 37: Bravo et al. 2016; 50: Aquino et al. 2007b; 52: Bravo et al. 2016; 53: Montenegro y Moya 2011; 56: Montenegro y Moya 2011; 62 Montenegro y Moya 2011; 76: Montenegro y Moya 2011. Requena.- 221: Saldaña y Saldaña 2011; 230: Voss y Fleck 2017; 233: Bardales-Alvites et al. 2017; 234: Fleck y Harder 2000, Voss y Fleck 2017; 239: Bardales-Alvites et al. 2017; 241: Amanzo 2006; 242: AMNH 98611-2; 243: Pérez-Peña et al. 2017; 245: Torres-Oyarce et al. 2017; 255: Amanzo 2006; 291: Jorge y Velazco 2006. Ucayali.- 268: MUSM 690-1; 285: Pérez-Peña et al. 2017; 287: Pacheco y Arias 2001; 289: Pacheco y Arias 2001.

Mazama americana

Alto Amazonas.- 246: Patterson y López-Wong 2014; 248: Patterson y LópezWong 2014; 256: Patterson y López-Wong 2014. Datem del Marañón.- 144: FMNH 88804-5. Loreto.- 55: Falcón-Ayapi et al. 2019, Pérez-Peña et al. 2012; 87: Aquino et al. 2014c, 172: FMNH 123029; 225: Pacheco y Pezo 1982; 228: FMNH 86899; 232: Pittet y Bennett 2014. Mariscal Ramón Castilla.- 54: Bravo et al. 2016; 71: Puertas et al. 2017; 99: Voss y Fleck 2017; 173: Mayor et al. 2015, 2016. Maynas.- 3: Pérez-Peña et al. 2019; 10: Pérez-Peña et al. 2019; 12: Pérez-Peña et al. 2019; 20: Aquino et al. 2014c; 38: AMNH 71739-41; 67: Gilmore 2005; 104: AMNH 73586; 109: Voss y Fleck 2017; 122: AMNH $98620-$ 3; 159: Aquino et al. 2007a, 2012b, Navarro y Terrones 2006; 169: MUSM 2723-36. Putumayo.- 37: Bravo et al. 2016; 50: Aquino et al. 2007b; 52: Bravo et al. 2016; 53: Montenegro y Moya 2011; 56: Montenegro y Moya 2011; 62: Montenegro y Moya 2011; 76: Montenegro y Moya 2011. Requena.- 221: Saldaña y Saldaña 2011; 230: Voss y Fleck 2017; 233: Bardales-Alvites et al. 2017; 234: Fleck y Harder 2000, Voss y Fleck 2017; 239: Bardales-Alvites et al. 2017; 241: Amanzo 2006; 261: Amanzo 2006; 291: Jorge y Velazco 2006; 292: Jorge y Velazco 2006. Ucayali.- 267: MUSM 686; 284: AMNH 98628; 287: Pacheco y Arias 2001; 289: Pacheco y Arias 2001.

Mazama nemorivaga

Alto Amazonas.- 246: Patterson y López-Wong 2014. Datem del Marañón.114: FMNH 88809. Loreto.- 49: KU 158257; 55: Falcón-Ayapi et al. 2019 , Pérez-Peña et al. 2012; 87: Aquino et al. 2014c; 211: AMNH 98624. Mariscal Ramón Castilla.- 54: Bravo et al. 2016; 71: Puertas et al. 2017; 173: Mayor et al. 2015. Maynas.- 3: Pérez-Peña et al. 2019; 10: Pérez-Peña et al. 2019; 12: Pérez-Peña et al. 2019; 20: Aquino et al. 2014c; 104: AMNH 73768; 109: Voss y Fleck 2017; 142: MUSM 16457-8; 159: Aquino et al. 2007a, 2012b, Navarro y Terrones 2006; 169: MUSM 2737-42. Putumayo.- 37: Bravo et al. 2016; 50: Aquino et al. 2007b; 52: Bravo et al. 2016; 76: Montenegro y Moya 2011. Requena.- 234: Voss y Fleck 2017; 239: Bardales-Alvites et al. 2017; 241: Amanzo 2006; 255: Amanzo 2006; 261: Amanzo 2006. Ucayali.277: MUSM 2166.

Sotalia fluviatilis

Loreto.- 188: Sittig et al. 2018; 194: McGuire 2010; 196: McGuire 2010; 199: McGuire 2010; 200: McGuire 2010; 202: McGuire 2010; 203: McGuire 2010; 204: McGuire 2010; 212: McGuire 2010; 251: McGuire 2010. Mariscal Ramón Castilla.- 54: Bravo et al. 2016. Putumayo.- 56: Montenegro y Moya 2011; 62: Montenegro y Moya 2011

Inia geoffrensis

Loreto.- 164: Sittig et al. 2018; 212: AMNH 188220, 235207; 232: McGuire y Aliaga-Rossel 2007. Mariscal Ramón Castilla.- 54: Bravo et al. 2016. Maynas.- 105: LACM 19589; 120: LACM 54440, 72146; 121: LACM 19590; 122: LACM 27073-5; 140: LACM 19588. Putumayo.- 37: Bravo et al. 2016; 56: Montenegro y Moya 2011; 62: Montenegro y Moya 2011. Requena.- 261: Amanzo 2006. 